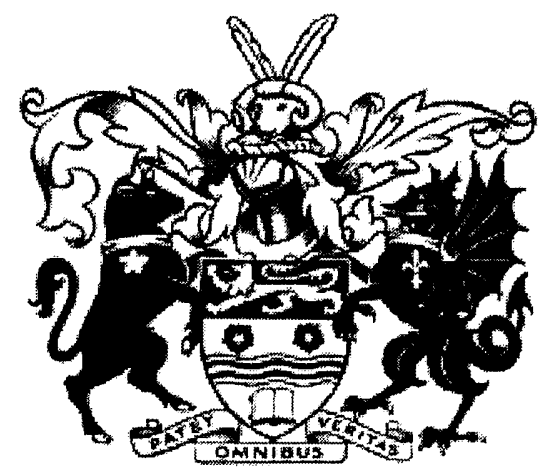

UNIVERSITY OF LANCASTER

\title{
Theory of Bilayer Graphene Spectroscopy
}

\section{Marcin Mucha-Kruczyński, M.Sc.}

Thesis submitted for the degree of Doctor of Philosophy

Under the supervision of:

Prof. Vladimir I. Fal'ko

Dr Edward McCann

December 9, 2010 
ProQuest Number: 11003506

All rights reserved

INFORMATION TO ALL USERS

The quality of this reproduction is dependent upon the quality of the copy submitted.

In the unlikely event that the author did not send a complete manuscript and there are missing pages, these will be noted. Also, if material had to be removed, a note will indicate the deletion.

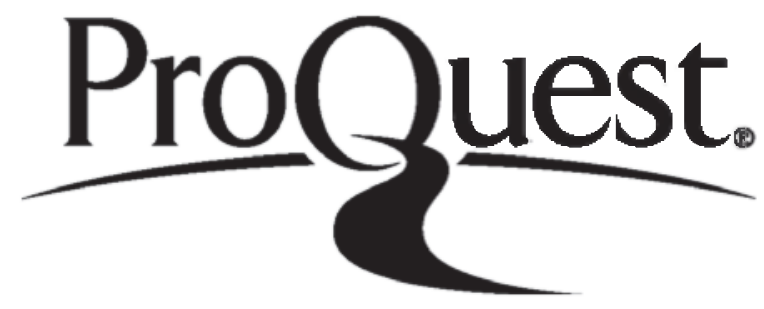

ProQuest 11003506

Published by ProQuest LLC (2018). Copyright of the Dissertation is held by the Author.

All rights reserved.

This work is protected against unauthorized copying under Title 17, United States Code Microform Edition (C) ProQuest LLC.

ProQuest LLC.

789 East Eisenhower Parkway

P.O. Box 1346

Ann Arbor, Ml $48106-1346$ 
This thesis is the author's own work, and has not been submitted in substantially the same form for the award of a higher degree elsewhere. The following parts of the thesis have been published as separate articles in peer-reviewed journals:

- Parts of Section 2.3 in: Electron-hole asymmetry and energy gaps in bilayer graphene, Semiconductor Science \& Technology 25, 033001 (2010);

- Parts of Chapter 3 in: Characterization of graphene through anisotropy of constant-energy maps in angle-resolved photoemission, Physical Review B 77, 195403 (2008);

- Parts of Chapter 4 in:

- On spectral properties of bilayer graphene: the effect of an SiC substrate and infrared magneto-spectroscopy, Journal of Physics: Condensed Matter 21, 344206 (2009);

- Influence of interlayer asymmetry on magnetospectroscopy of bilayer graphene, Solid State Communications 149, 1111 (2009);

- Parts of Chapter 5 in: Spectral features due to inter-Landau-level transitions in the Raman spectrum of bilayer graphene, Physical Review B 82, 045405 (2010). 


\begin{abstract}
In this thesis, we model theoretically spectra measured for bilayer graphene obtained using the angle-resolved photoemission spectroscopy, magneto-optical absorption spectroscopy and electronic Raman spectroscopy. The theories are based on the tight-binding description of the $\pi$ bands in the material. In particular, we concentrate on the comparison of the four-band model and its effective low-energy approximation neglecting the split high-energy bands, in the description of specific spectra. We demonstrate that both for monolayer and bilayer graphene, the observed anisotropy of angle-resolved photoelectron spectroscopy spectra reflects the electronic chirality in the system. However, for bilayer graphene, the influence of the nonchiral dimer states not captured within the effective approximation is significant and should not be neglected. We also show that the anisotropy of the constant-energy maps may be used to extract information about the magnitude and sign of interlayer coupling parameters and about symmetry breaking inflicted on a bilayer by the underlying substrate. We then determine selection rules and optical strengths of the inter-Landau-level excitations among any of the $\pi$ bands and including the physically most relevant symmetry-breaking parameters. We then present a self-consistent calculation of the interlayer asymmetry caused by an applied electric field in magnetic fields. We show how this asymmetry influences the Landau level spectrum in bilayer graphene and the observable inter-Landau level transitions when they are studied as a function of high magnetic field at fixed filling factor as measured experimentally. We also analyse the magneto-optical spectra of bilayer flakes in the photon-energy range corresponding to transitions between degenerate and split bands of bilayers. Finally, we investigate the contribution of the lowenergy electronic excitations toward the Raman spectrum of bilayer graphene for the incoming photon energy $\Omega \gg 1 \mathrm{eV}$. Using the four-band model, we de-
\end{abstract}


rive an effective scattering amplitude that can be incorporated into the two-band approximation and show that this amplitude is different from the contact interaction amplitude obtained within the two-band model alone. We then calculate the spectral density of the inelastic light scattering accompanied by the excitation of electron-hole pairs in bilayer graphene. In the absence of a magnetic field this contribution is constant and in doped structures has a threshold at twice the Fermi energy. In an external magnetic field, the dominant Raman-active modes are the $n_{-} \rightarrow n_{+}$inter-Landau-level transitions with crossed polarization of in/out photons. We estimate the quantum efficiency of a single $n_{-} \rightarrow n_{+}$transition in the magnetic field of $10 \mathrm{~T}$ as $I_{n_{-}-n_{+}} \sim 10^{12}$. 


\section{Acknowledgements}

The story of my PhD starts much before the official admission date to Lancaster University. However, through all of it, two people played dominant roles. The first is Prof. V. I. Fal'ko, who six years ago gave series of lectures during a summer school in a quite remote city of Jyväskylä. He later helped one of the students who attended that course to study at Lancaster University for a year as an exchange student. Three and a half years ago the student came back to Lancaster to start his PhD under Prof. Fal'ko's guidance. Without this guidance and his always-present fascination with physics, I would not have arrived to where I am now. The second person I would like to thank is my joint supervisor Dr Edward McCann, who more than once discussed and helped me understand those 'simple and obvious' details I was stuck at, whether as an undergrad or a finishing PhD student. Acknowledgements are also due to O. Kashuba, Y. Suprunenko and all those unnamed here, who through everyday discussions contributed towards my learning. I extend special thanks to my family, who always unanimously supported me in my decision to come to Lancaster. Last but not least, a thank you to Amie, who at times was probably the most interested of all involved in the finalisation of this thesis. 


\section{Table of Contents}

1 Introduction $\quad 1$

1.1 Graphene on paper and in the lab . . . . . . . . . . . . 1

1.2 Two layers: double the fun? . . . . . . . . . . . . 3

1.3 Thesis outline ....................... 4

2 The tight-binding approach and the resulting electronic structure 8

2.1 The crystal and reciprocal lattices . . . . . . . . . . . 8

2.2 The four-band tight-binding model for $\pi$ electrons . . . . . . . . . 11

2.2.1 Full momentum dependence . . . . . . . . . . . . 11

2.2.2 Approximation for hopping elements . . . . . . . . . 16

2.2.3 Symmetry breaking asymmetries in the on-site energies . 19

2.3 The effective two-band model . . . . . . . . . . . . . . . . 20

3 Angle-Resolved Photoemission Spectroscopy 24

3.1 ARPES as quantum Young's experiment . . . . . . . . . . . . 26

3.2 Monolayer Graphene . . . . . . . . . . . . . . . . . . . 30

3.3 Bilayer Graphene . . . . . . . . . . . . . . . . . 33

3.3.1 Low-energy spectrum: contribution of the two degenerate bands only ...................... 33

3.3.2 Contribution from the split bands . . . . . . . . . 34 
3.3.3 Influence of the symmetry breaking parameters on the ARPES spectra . . . . . . . . . . . . . . . . . . . . 39

3.3.4 Interference due to a finite interlayer distance $\ldots \ldots .40$

4 Magneto-Optical Spectroscopy $\quad 42$

4.1 Bilayer graphene in an external magnetic field . . . . . . . 45

4.1.1 Landau levels in the two-band model . . . . . . . . . 45

4.1.2 Landau levels in the four-band model . . . . . . . . . 46

4.1.3 Numerical treatment of the $\gamma_{3}$ coupling . . . . . . . 50

4.2 Magneto-optical selection rules and the absorption spectra . . . . 53

4.3 Magneto-Optical spectroscopy in charged bilayer graphene . . . . 56

4.3.1 Landau level spectrum in charged bilayer graphene: selfconsistent analysis of the interlayer asymmetry gap . . . . 56

4.3.2 Tracking a single inter-LL transition: low-energy interLandau level transitions . . . . . . . . . . . . . . 60

4.3.3 Magneto-optical spectra in charged bilayer: high-energy inter-Landau level transitions . . . . . . . . . . . . 63

5 Electronic Raman Spectroscopy $\quad 66$

5.1 The two-photon field and the electron-photon interaction . . . . 68

5.2 Theory of Raman scattering in graphene . . . . . . . . 70

5.2.1 Contribution of the contact interaction . . . . . . . . 71

5.2.2 Contribution of the two-step processes $\ldots \ldots \ldots \ldots 72$

5.2.3 The resulting Raman scattering amplitude . . . . . . . 73

5.3 ERS spectra in the absence of the magnetic field . . . . . . . 74

5.4 ERS spectra in quantizing magnetic fields . . . . . . . 76

6 Conclusions $\quad 81$

$\begin{array}{ll}\text { Bibliography } & 84\end{array}$ 


\section{List of Figures}

2.1 The real and reciprocal lattices . . . . . . . . . . . . . . 10

2.2 The band structure of bilayer graphene $\ldots \ldots \ldots \ldots$

2.3 The band structure around the valleys in the linear approximation 18

2.4 Influence of the symmetry-breaking parameters on the band struc-

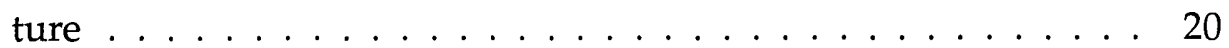

2.5 The electronic dispersion at very low energies $\ldots \ldots \ldots \ldots$

3.1 The ARPES spectra of monolayer graphene . . . . . . . . . 32

3.2 The ARPES spectra of bilayer graphene within the low-energy

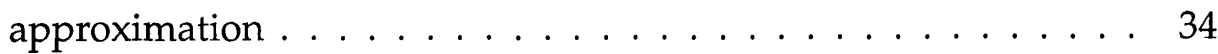

3.3 The ARPES spectra of bilayer graphene . . . . . . . . . . . 37

3.4 The influence of the signs of $\gamma_{1}$ and $\gamma_{3}$ on the ARPES spectra . . 38

3.5 The influence of the interlayer asymmetry on the ARPES spectra . 40

3.6 The influence of the interlayer spacing on the ARPES spectra . . . 41

4.1 Landau level structure of bilayer graphene . . . . . . . . . . . 49

4.2 Landau level structure of bilayer graphene at low energies and weak fields . . . . . . . . . . . . . . . . . . . . 52

4.3 Selection rules for the magneto-optical absorption . . . . . . . 56

4.4 Schematic of a 'biased bilayer' . . . . . . . . . . . . . 58 
4.5 Self-consistently calculated values of the interlayer asymmetry $u$ and corresponding LL spectra . . . . . . . . . . . . . . . 59

4.6 Energy of low-energy inter-LL excitations for $w=0 \ldots 61$

4.7 Energy of low-energy inter-LL excitations for $w=-100 \mathrm{meV} \ldots 62$

4.8 Examples of magneto-optical absorption spectra of bilayer graphene in a strong external magnetic field . . . . . . . . . . . . 64

5.1 Schematic depiction of the one and two-step ERS processes . . . . 69

5.2 Allowed inter-LL transitions and the low-energy ERS . . . . . . 78

5.3 Electronic contributions to the Raman spectra for different incoming beam energies . . . . . . . . . . . . . . . . 79 


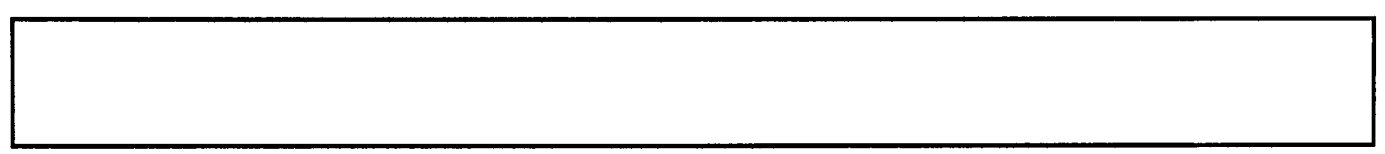

\section{List of Tables}

2.1 Summary of the vectors introduced for the real and reciprocal




$\Gamma_{\text {Chapter }} 1$

\section{Introduction}

\subsection{Graphene on paper and in the lab}

With many models in physics, it is much easier to conceive a Gedankenexperiment and analyse it on paper, rather than prepare a real-life experiment. It is definitely the case when imagining a single plane of carbon atoms arranged in a honeycomb (hexagonal) pattern. As a conceptual building block of graphite [1], this model has been used by theorists to explain this material's physical and chemical properties for more than sixty years [2-4]. It resurfaced now and again, especially with the discovery of fullerenes [5] and tremendous interest following the rediscovery of carbon nanotubes [6,7], both of which can be thought of as constructed from a layer of tightly arranged benzene rings. Somewhere along the way, the model got a name - graphene, signalling the presence of the planar $s p^{2}$ bonds between carbon atoms and emphasizing its importance in connection to graphite.

In the end, bulk graphite that was the reason for the Gedankenexperiment, had the main role in the real-life one. In 2004, Andre Geim's group at the University of Manchester, experimenting at the time with mechanical exfolation of layers from layered materials, isolated few-layer graphene films, including a single layer, from thin samples of highly-oriented pyrolytic graphite $[8,9]$. Sheets of carbon, one atom thick, have been shown to be stable under ambient 
environment and were successfully processed into devices allowing for the investigation of their transport properties. These were found promising for both fundamental and application-oriented research: they confirmed theoretically predicted gapless linear dispersion of the quasiparticles in the vicinity of the Fermi energy, electron mobility $\sim 10^{4} \frac{\mathrm{cm}^{2}}{\mathrm{~V}_{\mathrm{s}}}$, the mean free path of the order of tenths of micrometer, huge sustainable currents $>10^{8} \frac{\mathrm{A}}{\mathrm{cm}^{2}}$ and unusual sequence of plateaus in the Quantum Hall Effect associated with an additional electronic degree of freedom due to the symmetry of the crystal lattice $[8,10,11]$. Those first experiments attracted, therefore, a huge interest in the condensed matter community. In six years, their seminal Science paper, [8], has been cited more than 2800 times $^{1}$. The arXiv.org on-line archive alone lists over two thousand papers on widely defined graphene systems (monolayer, bilayer, few-layer films, nanoribbons, etc.) submitted since 2007. Multiple general [12-16] and more detailed reviews on synthesis [17, 18], optical [19], Raman [20,21] and photoemission [22] studies and electronic structure and transport [23-28] are already avialable in the literature.

Other than mechanical exfoliation, ways to obtain graphene were explored and advances in the epitaxial growth of carbon crystallites and layers on $\mathrm{SiC}$ and metallic substrates [29-31] were taken advantage of. Note that although in some of those cases prior to 2004 single layers of carbon atoms have been grown on a substrate, their properties (for example the electronic dispersion at the $K$ point) were dissimilar to monolayer graphene. Currently, after significant development, graphene-like layers can also be grown epitaxially on multiple substrates like SiC [32-34], $\mathrm{Ni}$ [35-38], Ir [39], Ru [40-42] or $\mathrm{Cu}$ [43]. On some of these, only monolayer or effectively monolayer-like decoupled layers can be grown while others allow for growth of few-layer graphene films in various stackings. The technology is already advanced enough to produce coverage areas of the

\footnotetext{
${ }^{1}$ according to the Thomson's ISI Web of Knowledge data
} 
order of square inch [43-48]. Reliable and detailed tools for characterisation of all graphene-like systems are necessary to evaluate their quality quickly and efficiently. At the same time, extensive knowledge of and about the materials is needed to design future devices and engineer new graphene-based systems. This thesis describes efforts undertaken to define a minimal theoretical model, allowing for such prediction, for one of the many materials from the graphene family, that is bilayer graphene.

\subsection{Two layers: double the fun?}

Bilayer graphene was first obtained with the scotch-tape technique, originally used to obtain monolayer graphene. A very 'messy' method, it yields at the final stage all kinds of graphene-based thin films with varying thicknesses. Some of them consist of two coupled layers of graphene. The most energetically favourable relative arrangement of those layers is the $A B$ (or Bernal) stacking [49], also found in crystalline graphite [1, 50]. McCann and Fal'ko showed that the low-energy electronic spectrum of such a system, relevant for transport experiments, is qualitatively different from the monolayer [51]. Most significantly, the interlayer coupling changes the dispersion from linear to quadratic. The quasiparticles now exhibit Berry phase of $2 \pi$ leading to, for example, weak localisation instead of weak anti-localisation as in the monolayer $[52,53]$. However, at least in the neutral system, the spectrum is still gapless. This last fact leads to the presence of a sixteenfold (including spin) degenerate Landau level positioned at zero energy (Fermi energy in the neutral structure) and yet another unusual sequencing of the plateaus in the Quantum Hall Effect as the plateau at $\sigma_{x y}=0$ is missing [54]. McCann and Fal'ko were also the first to point out the possibility of breaking the layer symmetry by applying an external electric field. This gap was first directly observed with the help of the angle-resolved photoemission by Ohta et al. [55]. Thus, bilayer graphene became technologi- 
cally relevant as one of the several options of introducing a gap in the electronic spectrum of graphene-based systems. On a more basic level, bilayer graphene introduced to solid state physics the unique notion of massive chiral fermions.

\subsection{Thesis outline}

As aforementioned, bilayer graphene, despite its name and origins, can definitely be considered a material on its own rights, rather than a poor cousin of (monolayer) graphene. As the experimental effort in its characterisation grows and first devices are built, it is desirable to construct a theoretical model, simple yet universal, capturing essential physics in wide scope of laboratory relevant situations. In this thesis, we probe the limits and capabilities of the tight-binding description of $\pi$ electrons in bilayer graphene in description of spectra obtained by using various spectroscopic techniques: angle-resolved photoemission spectroscopy, magneto-optical spectroscopy and electronic Raman spectroscopy. In particular, we concentrate on the comparison of the so called four-band and two-band models and the importance of the symmetry breaking on-site energies.

In this thesis, we model theoretically spectra of bilayer graphene as measured with various spectroscopic techniques: angle-resolved photoemission spectroscopy (ARPES), magneto-optical spectroscopy and electronic Raman spectroscopy (ERS). We search for the minimal model based on the tight-binding approach, which captures the essential physics in all of those cases. We therefore start with the introduction of the tight-binding model of $\pi$ electrons for graphene systems, presented in Chapter 2. We discuss in detail, the four-band model describing the $\pi$ bands within the whole Brillouin zone and the linear approximation valid only in the vicinity of its corners and define the symmetrybreaking parameters contained within the model. We also describe the effective two-band, low-energy approximation for the band structure of bilayer graphene, 
which is the start point for a significant part of the theoretical models postulated in the literature to describe the physics of bilayer graphene.

The choice of the spectroscopic methods under consideration has been mainly dictated by the developments in the experimental characterisation of graphene materials. The angle-resolved studies have been performed extensively on epitaxially grown monolayer graphene (e.g., $[34,35,39,56,57])$. Photoemission is one of the methods used, for example, to confirm the linearity and lack/presence of gaps in the electronic spectrum of graphene-like materials. The ARPES spectra of bilayer graphene have been used to demonstrate directly the presence of the gap in the electronic spectrum as the interlayer asymmetry has been introduced [55]. Our model [58], presented in Chapter 3, examines the angular distribution of the constant-energy maps of the ARPES intensity for monolayer and bilayer graphene. We show how these are related to the chirality of electrons in those systems. Afterwards, we show that for bilayer graphene, the anisotropy of the constant-energy maps may be used to extract information about the magnitude and sign of interlayer coupling parameters and about symmetry breaking inflicted on a bilayer by the underlying substrate.

The second spectroscopic method considered in this thesis, magneto-optical absorption spectroscopy, is one of the experimental tools employed to examine the electronic states in graphene systems in external magnetic fields [59-68]. In Chapter 4, we investigate some of the aspects of magneto-optical spectra from the theoretical point of view. We start with the description of the Landau level structure in bilayer graphene. We complete the thoery based on the two-band model $[69,70]$ and give selection rules as well as the optical strengths of the interLandau-level excitations taking into account all four $\pi$ bands and the physically most relevant asymmetries [71]. We then look closer at the experimental setup used to probe the Landau level structure in bilayer graphene and discuss the importance of the external gates used to vary the carrier density in the bilayer 
during the experiment. Building on the theory proposed for the case of no magnetic field [72], we then present a self-consistent calculation of the electricfield-induced interlayer asymmetry in magnetic fields and the resulting Landau level structure. We also analyse the magneto-optical spectra of bilayer flakes in the photon-energy range corresponding to transitions between degenerate and split bands of bilayers [73].

Finally, in Chapter 5, we turn towards Raman spectroscopy. Routinely used to characterise carbon materials, in graphene systems in particular, it provides information on, for example, the number of layers, domain sizes, doping levels, thermal conductivity and the structure of edges [20,21]. Here, we concentrate on relatively unexplored in graphene materials, purely electronic in origin, processes leading to inelastic scattering of light from the sample. The experimental approach focusing on such processes is often called electron Raman scattering/spectroscopy (ERS) [74]. We study the contribution of the low-energy electronic excitations toward the Raman spectrum of bilayer graphene for the incoming photon energy $\Omega \gg 1 \mathrm{eV}$ both with and without an external magnetic field [75]. Starting with the four-band tight-binding model, we derive an effective scattering amplitude that can be incorporated into the two-band approximation. We show that this effective scattering amplitude is different from the contact interaction amplitude obtained within the two-band model alone. We then calculate the spectral density of the inelastic light scattering accompanied by the excitation of electron-hole pairs in bilayer graphene. In the absence of a magnetic field, due to the parabolic dispersion of the low-energy bands in a bilayer crystal, this contribution is constant and in doped structures has a threshold at twice the Fermi energy. In an external magnetic field, the dominant Raman-active modes are the $n_{-} \rightarrow n_{+}$inter-Landau-level transitions with crossed polarization of in/out photons. We estimate the quantum efficiency of a single $n_{-} \rightarrow n_{+}$transition in the magnetic field of $10 \mathrm{~T}$ as $I_{n_{-} m_{+}} \sim 10^{-12}$, which may be 
experimentally observable.

We summarise the work presented in this thesis in Chapter 6. Based on Chapters 3, 4 and 5, we discuss the applicability of the two- and four-band models with respect to the electronic structure of bilayer graphene around the Fermi energy and prediction of experimental measurements. 


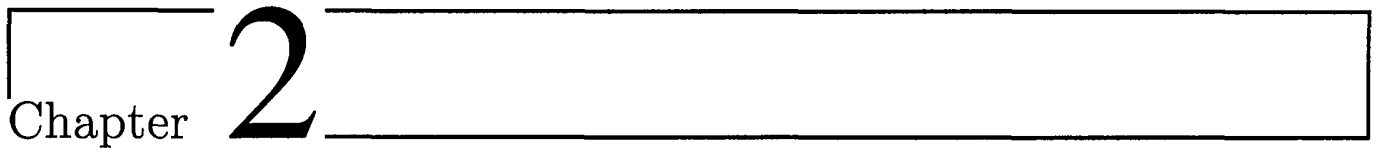

\section{The tight-binding approach and the resulting electronic structure}

In this chapter, we describe the crystal and reciprocal lattices of bilayer graphene. We also discuss briefly the symmetry of the crystal lattice. We then introduce the tight-binding model for $\pi$-electrons in bilayer graphene. We start with a general formulation valid for all points in the Brillouin zone and the resulting electronic structure. Next, we concentrate on the linear approximation of that model around the corners of the Brillouin zone. This tight-binding approach is a variation of the tight-binding model for monolayer graphene as developed historically for applications in the physics of graphite (then so called Slonczewski-Weiss-McClure model $[2-4,76])$. For an introduction to the tightbinding approach in carbon $s p^{2}$ materials, see [77] or [78]. In the following section, we introduce symmetry-breaking parameters which will later turn out to be very important when interpreting results of spectroscopic measurements. We conclude the chapter with the derivation of the effective low-energy, twoband Hamiltonian for bilayer graphene.

\subsection{The crystal and reciprocal lattices}

Bilayer graphene consists of two coupled graphene layers of carbon atoms (graphene monolayers) arranged in Bernal $(A B)$ stacking $[1,50]$. The unit cell 
contains four inequivalent carbon sites $A 1, B 1, A 2$, and $B 2$, where $A$ and $B$ denote two triangular sublattices in the same layer while 1 and 2 distinguish between the bottom and top layer, respectively. The real lattice of bilayer graphene is schematically shown in Fig. 2.1(a). The honeycomb lattice of the bottom and top layers has been drawn with red and black solid lines, respectively. The lattice constant $a$, that is the $A-A$ (or $B-B$ ) distance, marked in the figure with grey, equals $2.46 \AA$. This lattice constant derives from the benzene-ring structure and is the same in bilayer graphene as in monolayer graphene or graphite. The interlayer distance, $c_{0}$, is much greater than the nearest neighbour carbon-carbon distance $\frac{a}{\sqrt{3}} \AA$. X-ray reflectivity experiments and first-principles calculations performed for bilayer graphene epitaxialy grown on $\mathrm{SiC}$ [79], as well as first-principles calculations for bilayer in vacuum [80], lead to $c_{0} \approx 3.35 \AA$, as in graphite. We choose vectors $a_{1}$ and $a_{2}$ as our in-plane primitive lattice vectors and a rhombic unit cell as shown in Fig. 2.1(a) with a dashed blue line. Also shown in the figure are vectors $d_{1}, d_{2}$ and $d_{3}$, which can be used to express the distance between neighbouring in-plane carbon atoms. Eventually, we use vector $c_{0}=\left(0,0, c_{0}\right)$ to describe the thickness of the bilayer.

The corresponding reciprocal lattice is schematically presented in Fig. 2.1(b). It is two-dimensional, because bilayer graphene, although strictly speaking three-dimensional due to the interlayer spacing, is not periodic in the $z$ direction. The reciprocal unit vectors $b_{1}$ and $b_{2}$, related to $a_{1}$ and $a_{2}$ via the condition $b_{i} \cdot a_{j}=2 \pi \delta_{i j}$, are shown in darker blue. The Brillouin zone is a hexagon, marked in the figure with a dashed line. We denote two inequivalent corners of the Bril-

Table 2.1: Components of the vectors in the real and reciprocal lattices shown in Fig. 1 and used throughout the text.

\begin{tabular}{|c|c|c|c|c|c|c|c|c|c|}
\hline vector component & $a_{1}$ & $a_{2}$ & $d_{1}$ & $d_{2}$ & $d_{3}$ & $b_{1}$ & $b_{2}$ & $\boldsymbol{K}_{+}$ & $\boldsymbol{K}_{-}$ \\
\hline$x$ or $k_{x}$ & $\frac{a}{2}$ & $\frac{a}{2}$ & 0 & $\frac{a}{2}$ & $-\frac{a}{2}$ & $\frac{2 \pi}{a}$ & $\frac{2 \pi}{a}$ & $\frac{4 \pi}{3 a}$ & $-\frac{4 \pi}{3 a}$ \\
\hline$y$ or $k_{y}$ & $\frac{a \sqrt{3}}{2}$ & $-\frac{a \sqrt{3}}{2}$ & $\frac{a}{\sqrt{3}}$ & $-\frac{a}{2 \sqrt{3}}$ & $-\frac{a}{2 \sqrt{3}}$ & $\frac{2 \pi}{a \sqrt{3}}$ & $-\frac{2 \pi}{a \sqrt{3}}$ & 0 & 0 \\
\hline
\end{tabular}





Figure 2.1: (a) Schematic drawing of the bilayer graphene crystal lattice. The bottom (top) layer is depicted with red (black) solid lines. The real primitive lattice vectors are $a_{1}$ and $a_{2}$ and the unit cell is shown with dashed blue line. Grey line marks the lattice constant $a$. Vectors $d_{1}, d_{2}$, and $d_{3}$, are used to express the relative position of neighbouring carbon atoms. (b) The reciprocal lattice of bilayer graphene with the Brillouin zone shown with the dashed blue line and its two inequivalent corners (valleys) $K_{+}$and $K_{-}$. In contrast, the dotted blue line shows an alternative, rhombic unit cell in reciprocal space used briefly in Chapter 3. The orange dashed line shows the high-symmetry directions for which the band structure in Fig 2.2 is shown.

louin zone (later also called valleys) as $K_{+}$[at the position $\left.K_{+}=(4 \pi / 3 a, 0)\right]$ and $K_{-}$[at the position $\left.K_{-}=(-4 \pi / 3 a, 0)\right]$ and reserve index $\xi= \pm$ to distinguish between them in further discussions.

The real primitive lattice vectors $a_{1}$ and $a_{2}$, reciprocal primitive vectors $b_{1}$ and $b_{2}$, nearest neighbour vectors $d_{i}$, as well as the coordinates of the valley $K_{\xi}$ are repeatedly used throughout the remaining parts of the thesis. For convenience, all aforementioned vectors and their components in their respective space have been summarised in Table 2.1. 


\subsection{The four-band tight-binding model for $\pi$ elec- trons}

\subsubsection{Full momentum dependence}

Let us consider an infinite sheet of bilayer graphene. For the origin of the coordinate system, we choose the centre of a unit cell (position of the $B 1-A 2$ dimer, at the point halfway between the layers) and denote by $\boldsymbol{r}$ and $\boldsymbol{R}_{0}$ the position vector and a vector pointing to the centre of another unit cell (one of $N$ in total), respectively. We reserve symbol $\boldsymbol{R}_{i}$ to represent a vector pointing from the centre of a unit cell to the atomic site $i$ ( $i$ then stands for $A 1, B 1, A 2$ or $B 2$ ) in this unit cell. We assume periodic boundary conditions and construct a basis of functions $\phi_{k, i}(r)$ built up from the $\pi$-orbitals $\varphi(\mathbf{r})$ of carbon atoms in site $i$,

$$
\begin{aligned}
& \phi_{k, A 1}(\boldsymbol{r})=\frac{1}{\sqrt{N}} \sum_{R_{0}} e^{i \boldsymbol{k} \cdot\left(\boldsymbol{R}_{0}-\boldsymbol{d}_{1}-\frac{c_{0}}{2}\right)} \varphi\left(\boldsymbol{r}-\boldsymbol{R}_{0}+\boldsymbol{d}_{1}+\frac{\boldsymbol{c}_{0}}{2}\right), \\
& \phi_{\boldsymbol{k}, B 1}(\boldsymbol{r})=\frac{1}{\sqrt{N}} \sum_{\boldsymbol{R}_{0}} e^{i \boldsymbol{k} \cdot\left(\boldsymbol{R}_{0}-\frac{c_{0}}{2}\right)} \varphi\left(\boldsymbol{r}-\boldsymbol{R}_{0}+\frac{\boldsymbol{c}_{0}}{2}\right), \\
& \phi_{k, A 2}(r)=\frac{1}{\sqrt{N}} \sum_{R_{0}} e^{i k \cdot\left(R_{0}+\frac{c_{0}}{2}\right)} \varphi\left(r-R_{0}-\frac{c_{0}}{2}\right), \\
& \phi_{\boldsymbol{k}, B 2}(\boldsymbol{r})=\frac{1}{\sqrt{N}} \sum_{\boldsymbol{R}_{0}} e^{i \boldsymbol{k} \cdot\left(\boldsymbol{R}_{0}+d_{1}+\frac{c_{0}}{2}\right)} \varphi\left(\boldsymbol{r}-\boldsymbol{R}_{0}-\boldsymbol{d}_{1}-\frac{\boldsymbol{c}_{0}}{2}\right),
\end{aligned}
$$

where $k$ is a two-dimensional electron wave vector.

The electron wave function $\Psi_{j}(r)$, corresponding to the energy eigenvalue $\epsilon_{j}(k)$ of an electron with wave vector $k$, is a linear combination of functions in Eq. (2.1),

$$
\Psi_{j}(r)=\sum_{i} C_{i j} \phi_{k, i}(r) .
$$

It is easy to see that $\Psi_{j}(r)$ satisfies Bloch's theorem, as we have

$$
\Psi_{j}\left(r+m a_{1}+n a_{2}\right)=\sum_{i} C_{i j} \phi_{k, i}\left(r+m a_{1}+n a_{2}\right)=e^{i k \cdot\left(m a_{1}+n a_{2}\right)} \Psi_{j}(r) .
$$


By using the variational principle, we arrive with the matrix equation for the column vector $\psi_{j}$ of the coefficients $C_{i j}$,

$$
\hat{\boldsymbol{H}} \psi_{j}=\epsilon_{j}(k) \hat{\boldsymbol{S}} \psi_{j}
$$

To explicitly write down the form of the Hamiltonian operator $\hat{\boldsymbol{H}}$ and the overlap operator $\hat{S}$, we choose the order of the basis functions from Eq. (2.1) to be

$$
\phi_{k}(r)=\left(\phi_{k, A 1}(r), \phi_{k, B 2}(r), \phi_{k, A 2}(r), \phi_{k, B 1}(r)\right)^{T}
$$

We assume that the interaction between two carbon atoms depends only on their distance. Also, for a carbon atom on site $i$, we take into account only its interaction with the closest carbon atoms on sites $j$. We make an exception for the interaction of an atom on site $i$ with another on site $i$, where we include the influence of next-nearest neighbour of the same kind. The phase factor resulting from a summation over nearest neighbours can, for any carbon atom, be written in terms of the vectors $\boldsymbol{d}_{1}, \boldsymbol{d}_{2}$ and $\boldsymbol{d}_{3}$. We define the geometrical factor $f(\boldsymbol{k})$,

$$
f(\boldsymbol{k}) \equiv \sum_{i=1}^{3} e^{i k \cdot d_{i}}=e^{i \frac{k y a}{\sqrt{3}}}+2 e^{-i \frac{k y a}{2 \sqrt{3}}} \cos \frac{k_{x} a}{2} .
$$


As a result, the full matrix form of the operators $\hat{H}$ and $\hat{S}$ is ${ }^{1}$ :

$$
\begin{aligned}
\hat{\boldsymbol{H}} & =\left(\begin{array}{cccc}
\epsilon_{A 1}-\gamma_{n}|f(k)|^{2} & -\gamma_{3} f^{*}(\boldsymbol{k}) & \gamma_{4} f(k) & -\gamma_{0} f(k) \\
-\gamma_{3} f(k) & \epsilon_{B 2}-\gamma_{n}|f(k)|^{2} & -\gamma_{0} f^{*}(k) & \gamma_{4} f^{*}(k) \\
\gamma_{4} f^{*}(k) & -\gamma_{0} f(k) & \epsilon_{A 2}-\gamma_{n}|f(k)|^{2} & \gamma_{1} \\
-\gamma_{0} f^{*}(k) & \gamma_{4} f(k) & \gamma_{1} & \epsilon_{B 1}-\gamma_{n}|f(k)|^{2}
\end{array}\right) ; \\
\hat{\boldsymbol{S}} & =\left(\begin{array}{cccc}
1 & 0 & 0 & s_{0} f(k) \\
0 & 1 & s_{0} f^{*}(k) & 0 \\
0 & s_{0} f(k) & 1 & s_{1} \\
s_{0} f^{*}(k) & 0 & s_{1} & 1
\end{array}\right) .
\end{aligned}
$$

In the above, we introduced several parameters into the model as a description of the strength of interactions between carbon atoms. In this, we mostly follow the Slonczewski-Weiss-McClure model developed for bulk graphite [2$4,76]$ (for a review see [50]). The on-site energies $\epsilon_{i}$, couplings $\gamma_{j}$ and overlap

\footnotetext{
${ }^{1}$ We neglect in Eq. (2.6a) a factor of $3 \gamma_{n}$ appearing on the diagonal as it only leads to a shift of zero on the energy scale.
} 
parameters $s_{l}$ used in Eq. (2.6) are given by:

$$
\begin{aligned}
\epsilon_{i} & \equiv\left\langle\varphi\left(\boldsymbol{r}-\boldsymbol{R}_{0}-\boldsymbol{R}_{i}\right)|\hat{\boldsymbol{H}}| \varphi\left(\boldsymbol{r}-\boldsymbol{R}_{0}-\boldsymbol{R}_{i}\right)\right\rangle \\
\gamma_{0} & \equiv-\left\langle\varphi\left(\boldsymbol{r}-\boldsymbol{R}_{0}-\boldsymbol{R}_{A 1}\right)|\hat{\boldsymbol{H}}| \varphi\left(\boldsymbol{r}-\boldsymbol{R}_{0}-\boldsymbol{R}_{A 1}-\boldsymbol{d}_{i}\right)\right\rangle \equiv \\
& \equiv-\left\langle\varphi\left(\boldsymbol{r}-\boldsymbol{R}_{0}-\boldsymbol{R}_{A 2}\right)|\hat{\boldsymbol{H}}| \varphi\left(\boldsymbol{r}-\boldsymbol{R}_{0}-\boldsymbol{R}_{A 2}-\boldsymbol{d}_{i}\right)\right\rangle \\
\gamma_{1} & \equiv\left\langle\varphi\left(\boldsymbol{r}-\boldsymbol{R}_{0}-\boldsymbol{R}_{B 1}\right)|\hat{\boldsymbol{H}}| \varphi\left(\boldsymbol{r}-\boldsymbol{R}_{0}-\boldsymbol{R}_{A 2}\right)\right\rangle \\
\gamma_{3} & \equiv-\left\langle\varphi\left(\boldsymbol{r}-\boldsymbol{R}_{0}-\boldsymbol{R}_{A 1}\right)|\hat{\boldsymbol{H}}| \varphi\left(\boldsymbol{r}-\boldsymbol{R}_{0}-\boldsymbol{R}_{A 1}+\boldsymbol{d}_{i}-\boldsymbol{c}_{0}\right)\right\rangle \\
\gamma_{4} & \equiv\left\langle\varphi\left(\boldsymbol{r}-\boldsymbol{R}_{0}-\boldsymbol{R}_{A 1}\right)|\hat{\boldsymbol{H}}| \varphi\left(\boldsymbol{r}-\boldsymbol{R}_{0}-\boldsymbol{R}_{A 1}-\boldsymbol{d}_{1}-\boldsymbol{c}_{0}\right)\right\rangle \equiv \\
& \equiv\left\langle\varphi\left(\boldsymbol{r}-\boldsymbol{R}_{0}-\boldsymbol{R}_{B 1}\right)|\hat{\boldsymbol{H}}| \varphi\left(\boldsymbol{r}-\boldsymbol{R}_{0}-\boldsymbol{R}_{B 1}-\boldsymbol{d}_{1}-\boldsymbol{c}_{0}\right)\right\rangle \\
\gamma_{n} & \equiv\left\langle\varphi\left(\boldsymbol{r}-\boldsymbol{R}_{0}-\boldsymbol{R}_{i}\right)|\hat{\boldsymbol{H}}| \varphi\left(\boldsymbol{r}-\boldsymbol{R}_{0}-\boldsymbol{R}_{i}+\boldsymbol{d}_{3}-\boldsymbol{d}_{2}\right)\right\rangle \\
s_{0} & \equiv\left\langle\varphi\left(\boldsymbol{r}-\boldsymbol{R}_{0}-\boldsymbol{R}_{A 1}\right) \mid \varphi\left(\boldsymbol{r}-\boldsymbol{R}_{0}-\boldsymbol{R}_{A 1}-\boldsymbol{d}_{i}\right)\right\rangle \equiv \\
& \equiv\left\langle\varphi\left(\boldsymbol{r}-\boldsymbol{R}_{0}-\boldsymbol{R}_{A 2}\right) \mid \varphi\left(\boldsymbol{r}-\boldsymbol{R}_{0}-\boldsymbol{R}_{A 2}-\boldsymbol{d}_{i}\right)\right\rangle \\
s_{1} & \equiv\left\langle\varphi\left(\boldsymbol{r}-\boldsymbol{R}_{0}-\boldsymbol{R}_{B 1}\right) \mid \varphi\left(\boldsymbol{r}-\boldsymbol{R}_{0}-\boldsymbol{R}_{A 2}\right)\right\rangle
\end{aligned}
$$

The diagonal terms $\epsilon_{i}$ denote the on-site energy of the electron at the carbon atom in site $i$. In the first approximation, they are equal to the energy of an electron in the $2 p_{z}$ orbital of a carbon atom. This energy is modified as carbon atoms bond together to form the lattice. However, in an ideal and charge-neutral bilayer this on-site energy is approximately the same for each site in the lattice. In this case, we can take it to be zero and define our energy scale relatively to this point. More complicated situations in which the symmetry between the atomic sites has been broken are discussed in Sec. 2.2.3.

The parameters $\gamma_{j}$ describe the strength of the coupling between a specific pair of carbon atoms. The constant $\gamma_{0}$ denotes the coupling between the nearest neighbours ( $A 1 \leftrightarrow B 1$ and $A 2 \leftrightarrow B 2$ ). The parameter $\gamma_{1}$ describes the direct interlayer coupling $A 2 \leftrightarrow B 1$. The $\gamma_{3}$ coupling represents the interlayer interaction between the nearest $A 1$ and $B 2$ atoms, whereas $\gamma_{4}$ characterizes the interlayer 




Figure 2.2: The band structure of bilayer graphene resulting from eq. (2.4) with the Hamiltonian and overlap matrices $\hat{H}$ and $\hat{S}$ as in eq. (2.6) presented for highsymmetry directions in the Brillouin zone as shown in Fig 2.1(b). The values of the parameters used are: $\gamma_{0}=3.1 \mathrm{eV}, \gamma_{1}=0.4 \mathrm{eV}, \gamma_{3}=0.15 \mathrm{eV}, \gamma_{4}=0.1 \mathrm{eV}$, $\gamma_{n}=0.05 \mathrm{eV}, s_{0}=0.1, s_{1}=-0.05, \epsilon_{i}=0$ for all $i$, and $a=2.46 \AA$. The range of energies important in experimental setups modelled theoretically in this work is shown on red background.

coupling between the nearest $A 1$ and $A 2$, as well as $B 1$ and $B 2$ atoms. The last coupling, $\gamma_{n}$, describes the interaction of the in-plane next-nearest neighbours.

The overlap integrals $s_{l}$ take into account the fact that our $\pi$ orbitals do not span an orthogonal basis set. We only included here the overlap $s_{0}$ between two nearest neighbour atoms and the overlap $s_{1}$ between the $A 2$ and $B 1$ sites where atoms are directly above/below each other. Due to their small value, in most of the situations under consideration in this thesis, even these two overlap integrals are neglected.

The electronic band structure resulting from eq. (2.4) with the Hamiltonian and overlap matrices $\hat{H}$ and $\hat{S}$ as in eq. (2.6) is shown in Fig. 2.2. We see two conduction and two valence bands. The lower conduction band and the upper valence band touch exactly at the $K$ point. The position of this touching point at the energy scale denotes half-filling of the bands and is called the neutrality 
point. It is usually treated as the zero of the energy scale. In the neutral structure with the valence bands completely filled, the Fermi surface consists only of points. Any shift of the chemical potential results in the creation of separate Fermi lines around each of the $K$ points. Due to this behaviour of the Fermi surface, the $K$ points are often refered to as valleys. We point out that for most experiments and potential applications, only the part of the dispersion relatively close to the neutrality point $(\sim 1 \mathrm{eV})$ is important and interesting. Indeed, a proper description of this part of the band structure forms the basis for the understanding and theoretical modelling of spectroscopic experiments presented in this thesis. We will, therefore, investigate it in more detail in the following sections.

\subsubsection{Approximation for hopping elements}

We now want to look closer at the electronic dispersion for energies relevant to most experiments, that is, the energies of up to $\sim 1 \mathrm{eV}$ from the neutrality point. This range of energies is marked with red background in Fig. 2.2. For such energy, the relevant regions in momentum space are the vicinities of the six corners of the Brillouin zone. To describe electronic dispersion around a local minima at the $K$ points, we shift the coordinate system in reciprocal (momentum) space from the $\Gamma$ point to the $K_{\xi}$ point. We write the electron wave vector as $k=\boldsymbol{K}_{\xi}+\frac{p}{\hbar}$, where the electronic momentum $p$ is now measured from the centre of the valley $K_{\xi}$. The geometrical factor $f(k)$ from eq. (2.5), expanded up to the second order in $\boldsymbol{p}$, reads

$$
f(k) \approx-\frac{\sqrt{3} a}{2 \hbar}\left(\xi p_{x}-i p_{y}\right)+\frac{a^{2}}{8 \hbar^{2}}\left(p_{x}+i p_{y}\right)^{2}
$$

We introduce some new parameters, namely velocities $v=\frac{\sqrt{3} a \gamma_{0}}{2 \hbar}, v_{3}=\frac{\sqrt{3} a \gamma_{3}}{2 \hbar}$ and $v_{4}=-\frac{\sqrt{3} a \gamma_{4}}{2 \hbar}$, constant $\eta=\frac{a^{2} \gamma_{0}}{8 \hbar^{2}} \equiv \frac{v^{2}}{6 \gamma_{0}}$, as well as operators, $\hat{\pi}=p_{x}+i p_{y}$ and 
$\hat{\pi}^{\dagger}=p_{x}-i p_{y}$. We also neglect at this stage the overlap integrals $s_{0}$ and $s_{1}$, and hence obtain only a unit matrix on the right hand side of Eq. (2.4). Our basis now consists of 8 functions $\phi_{p, \xi, i}(r)$ (4 for each valley). However, for the cases considered in this thesis the valleys can be regarded as independent (we do not consider any valley-connecting processes) and it is usually enough to explicitly write down only the electronic Hamiltonian $\hat{\boldsymbol{H}}_{\xi}$ for one valley $K_{\xi}$,

$$
\hat{\boldsymbol{H}}_{\xi}=\xi\left(\begin{array}{cccc}
0 & v_{3} \hat{\pi} & v_{4} \hat{\pi}^{\dagger} & v \hat{\pi}^{\dagger} \\
v_{3} \hat{\pi}^{\dagger} & 0 & v \hat{\pi} & v_{4} \hat{\pi} \\
v_{4} \hat{\pi} & v \hat{\pi}^{\dagger} & 0 & \xi \gamma_{1} \\
v \hat{\pi} & v_{4} \hat{\pi}^{\dagger} & \xi \gamma_{1} & 0
\end{array}\right)-\eta\left(\begin{array}{cccc}
\frac{6 \gamma_{n}}{\gamma_{0}} p^{2} & \frac{v_{3}}{v}\left(\hat{\pi}^{\dagger}\right)^{2} & \frac{v_{4}}{v} \hat{\pi}^{2} & \hat{\pi}^{2} \\
\frac{v_{3}}{v} \hat{\pi}^{2} & \frac{6 \gamma_{n}}{\gamma_{0}} p^{2} & \left(\hat{\pi}^{\dagger}\right)^{2} & \frac{v_{4}}{v}\left(\hat{\pi}^{\dagger}\right)^{2} \\
\frac{v_{4}}{v}\left(\hat{\pi}^{\dagger}\right)^{2} & \hat{\boldsymbol{\pi}}^{2} & \frac{6 \gamma_{n}}{\gamma_{0}} p^{2} & 0 \\
\left(\hat{\pi}^{\dagger}\right)^{2} & \frac{v_{4}}{v} \hat{\pi}^{2} & 0 & \frac{6 \gamma_{n}}{\gamma_{0}} p^{2}
\end{array}\right) .
$$

In the Hamiltonian above, we have for now neglected the on site energies $\epsilon_{i}$, which are discussed in detail in Sec. 2.2.3. The order of the basis functions is ${ }^{2}$ $\left(\phi_{+, A 1}, \phi_{+, B 2}, \phi_{+, A 2}, \phi_{+, B 1}\right)^{T}$ in the $K_{+}$and $\left(\phi_{-, B 2}, \phi_{-, A 1}, \phi_{-, B 1}, \phi_{-, A 2}\right)^{T}$ in the $K_{-}$valley.

The same Hamiltonian can be obtained using the ' $\mathbf{k} \cdot \mathbf{p}$ ' approximation (see for example [81, 82] for detailed derivation). In this scheme, as a basis set we use functions $\tilde{\phi}_{p, \xi, i}(r)$ constructed from $\phi_{k, i}(r)$, eq. (2.1), calculated exactly in the centre of the valley $K_{\xi}$ and a plane wave envelope function which varies slowly at the distance of the order of the lattice constant $a[83,84]$ :

$$
\tilde{\phi}_{\boldsymbol{p}, \xi, i}(\boldsymbol{r}) \equiv e^{\frac{i}{\hbar} p \cdot r} \phi_{\boldsymbol{K}_{\xi, i}}(\boldsymbol{r}) \equiv \frac{1}{\sqrt{N}} e^{\frac{i}{\hbar} p \cdot r} \sum_{\boldsymbol{R}_{0}} e^{i \boldsymbol{K}_{\xi} \cdot\left(\boldsymbol{R}_{0}+\boldsymbol{R}_{i}\right)} \varphi\left(\boldsymbol{r}-\boldsymbol{R}_{0}-\boldsymbol{R}_{i}\right) .
$$

Comparing functions $\tilde{\phi}_{p, \xi, i}(r)$ and $\phi_{K_{\xi}+\frac{p}{\hbar}, i}(r)$, we can intuitively see why the Hamiltonians in both approximations take the same form. Both functions take similar values for $\boldsymbol{r} \approx \boldsymbol{R}_{0}-\boldsymbol{R}_{i}$, whereas in other regions the $\pi$ orbital $\varphi\left(\boldsymbol{r}-\boldsymbol{R}_{0}-\boldsymbol{R}_{i}\right)$ ensures that they both quickly decay, rendering the phase factors unimportant.

The electronic band structure resulting from the Hamiltonian (2.9) is shown

\footnotetext{
${ }^{2}$ For brevity, we omit the momentum index $p$ and explicit dependence of the basis functions $\phi_{p, \xi, i}$ on $r$.
} 
a)



K. valley b)



$K_{+}$valley

Figure 2.3: The band structure of bilayer graphene in the vicinity of both a) $K_{-}$and b) $K_{+}$valleys, obtained within the linear approximation, eq. (2.9). The values of the parameters used are: $\gamma_{0}=3.1 \mathrm{eV}, \gamma_{1}=0.4 \mathrm{eV}, \gamma_{3}=0.2 \mathrm{eV}, \gamma_{4}=0.1 \mathrm{eV}$, $\gamma_{n}=0.1 \mathrm{eV}, a=2.46 \AA$. No on site asymmetries are included (that is, $\epsilon_{i}=0$ for all i). For both valleys, the low-energy and high-energy bands are shown in yellow and red, respectively.

for both valleys in Fig. 2.3 for the energy range $3.5 \gamma_{1}$ away from the neutrality point. On this scale, all bands look approximately parabolic very close to the center of the valley and linear further away. The former is not exactly true for the bands shown in yellow (later referred to as the low-energy bands), as shown in Sec. 2.3. The bands marked in red (in what follows called the high-energy or split bands) are shifted away from the neutrality point by approximately the interlayer coupling, $\gamma_{1} \sim 0.4 \mathrm{eV}[55,85-92]$ in each direction. The velocity $v \sim 10^{6} \mathrm{~m} / \mathrm{s}[87-89,92]$ determines the slope of the linear parts of the bands. The isoenergetic lines create circles, which in the case of the low-energy bands are trigonally warped. This warping is the effect of the velocity $v_{3} \sim 0.1 v[89,92]$ as well as terms quadratic in the momentum $p$. The remaining velocity, $v_{4}$, breaks the electron-hole symmetry. It renormalizes somewhat the slopes of the linear parts and its effect is opposite in the conduction and valence bands. The next-nearest neighbour coupling $\gamma_{n}$ also breaks the electron-hole symmetry. The parameters $v, \gamma_{1}$ and $v_{3}$ are the most important in the description of the electronic dispersion around the valleys. At low energies, the deviation from the electron-hole symmetric situation is negligible for most cases. Also, it is 
difficult to experimentally separate the contributions of $\gamma_{4}, \gamma_{n}$ or even $s_{0}$ to the electron-hole asymmetry.

\subsubsection{Symmetry breaking asymmetries in the on-site energies}

Up to this point, we considered the kinetic energies of electrons on different atomic sites (the terms $\epsilon_{i}$ in Eq. (2.7a)) to be equal. In other words, all the carbon atoms in the lattice were chemically equivalent. However, that is obviously not the case as the environment of the dimer atoms, $B 1$ and $A 2$, is definitely different than the surroundings of the atoms $A 1$ and $B 2$. In general, three parameters are needed to account for differences between our four atomic sites. The (not unique) definitions we use here are:

$$
\begin{gathered}
\Delta_{A B}=\frac{1}{2}\left[\left(\epsilon_{A 1}+\epsilon_{A 2}\right)-\left(\epsilon_{B 1}+\epsilon_{B 2}\right)\right] ; \\
\Delta=\frac{1}{2}\left[\left(\epsilon_{A 1}+\epsilon_{B 2}\right)-\left(\epsilon_{B 1}+\epsilon_{A 2}\right)\right] ; \\
u=\frac{1}{2}\left[\left(\epsilon_{A 1}+\epsilon_{B 1}\right)-\left(\epsilon_{A 2}+\epsilon_{B 2}\right)\right] ; \\
\epsilon_{A 1}=\frac{1}{2}\left(u+\Delta+\Delta_{A B}\right) ; \quad \epsilon_{B 1}=\frac{1}{2}\left(u-\Delta-\Delta_{A B}\right) ; \\
\epsilon_{A 2}=\frac{1}{2}\left(-u-\Delta+\Delta_{A B}\right) ; \quad \epsilon_{B 2}=\frac{1}{2}\left(-u+\Delta-\Delta_{A B}\right) ;
\end{gathered}
$$

Then, $\Delta_{A B}$ describes the difference between on-site energies of $A$ and $B$ sublattice sites on each layer. We call it intralayer asymmetry. It may be influenced, especially in the bottom layer, by the underlying substrate. The next parameter, $\Delta$, accounts for an energy difference between dimer and non-dimer sites. Finally, $u$ characterizes the interlayer asymmetry between the two layers. This asymmetry can be significantly changed by doping the sample [55] or even continously varied with external gates [93]. This effect is discussed in more depth in Sec. 4.3.1. To show the influence of each of the asymmetries on the band structure, we add them separately to the tight-binding model and plot in Fig. 2.4 cuts through 

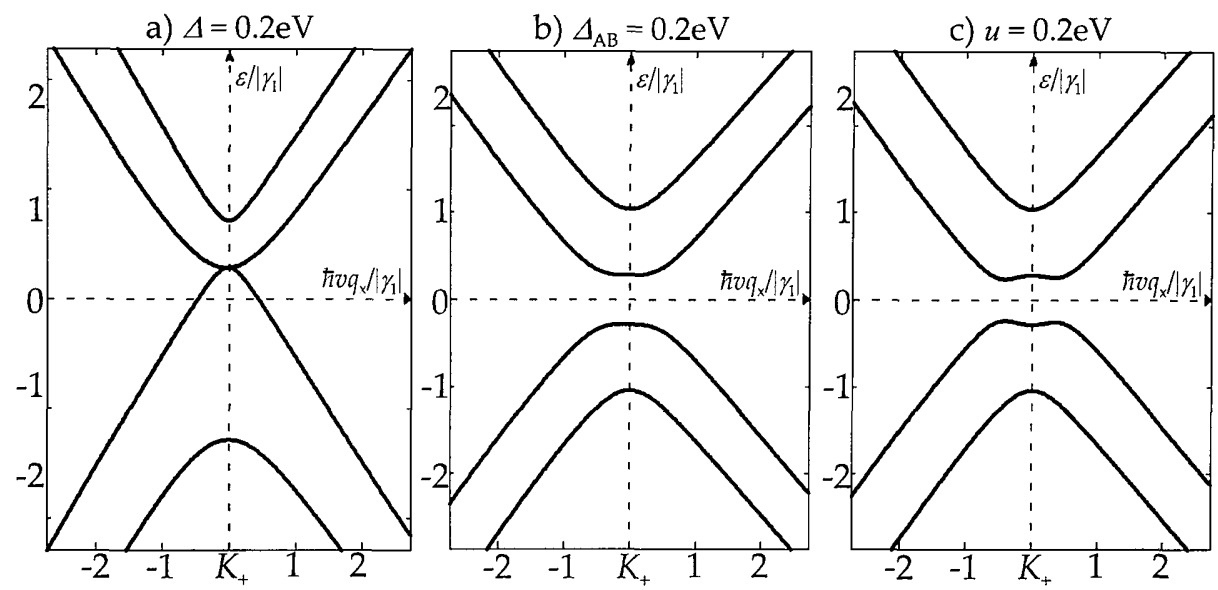

Figure 2.4: Cuts through the electronic dispersion around the $K_{+}$valley along the $p_{x}$ axis in the presence of a) $\left.\left.\Delta=0.2 \mathrm{eV}, \mathrm{b}\right) \Delta_{A B}=0.2 \mathrm{eV}, \mathrm{c}\right) u=0.2 \mathrm{eV}$. For illustrative purposes, we use large values of asymmetries. In reality, only the interlayer asymmetry $u$ can be of the order of $100 \mathrm{meV}[94,95]$. The values of other parameters used are: $\gamma_{0}=3 \mathrm{eV}, \gamma_{1}=0.35 \mathrm{eV}, \gamma_{3}=0.15 \mathrm{eV}, \gamma_{4}, \gamma_{n}=0 \mathrm{eV}$, $a=2.46 \AA$.

the electronic dispersion around the $K_{+}$point along the $p_{x}$ axis. The intralayer asymmetry does not open any gaps in the electronic spectrum but breaks the electron-hole symmetry. The dimer/non-dimer asymmetry $\triangle_{A B}$ opens a gap and preserves the electron-hole symmetry. The interlayer asymmetry $u$ preserves the electron-hole symmetry and also leads to the opening of a gap in the spectrum. However, we point out the characteristic 'Mexican-hat-like' features in the shape of the low-energy bands in the vicinity of the gap.

\subsection{The effective two-band model}

In this section, we derive an effective low-energy Hamiltonian describing the two degenerate bands in the close vicinity of the valley $K_{\xi}$. This analysis was first performed by McCann and Fal'ko [51] and proved extremely useful in describing the low-energy properties of bilayer graphene (see for example $[51,52,54,69,96,97])$. For a detailed discussion of this effective two-band approximation, see $[98,99]$. For simplicity, we take as the starting point the 
eigenproblem for the Hamiltonian in linear approximation, eq. (2.9), containing only the most important parameters $v, \gamma_{1}$ and $v_{3}$, and terms up to linear in the momentum $\boldsymbol{p}$. We also introduce the Pauli matrices $\sigma_{x}, \sigma_{y}$ and $\sigma_{z}$, and write the eigenproblem for the Hamiltonian as

$$
\left(\begin{array}{cc}
\xi v_{3}\left(\sigma_{x} p_{x}-\sigma_{y} p_{y}\right) & \xi v(\boldsymbol{\sigma} \cdot \boldsymbol{p}) \\
\xi v(\boldsymbol{\sigma} \cdot \boldsymbol{p}) & \sigma_{x} \gamma_{1}
\end{array}\right)\left(\begin{array}{l}
\psi_{1} \\
\psi_{2}
\end{array}\right)=\epsilon\left(\begin{array}{l}
\psi_{1} \\
\psi_{2}
\end{array}\right)
$$

where $\psi_{1}$ and $\psi_{2}$ denote two-component vectors which form together the fourcomponent electronic eigenstate $\psi$ from (2.4). Let us use the second row of (2.11) to express $\psi_{2}$ in terms of $\psi_{1}$ and $\epsilon$ and substitute it into the first row to obtain,

$$
\xi v_{3}\left(\sigma_{x} p_{x}-\sigma_{y} p_{y}\right) \psi_{1}+v^{2}(\sigma \cdot p)\left[\epsilon-\sigma_{x} \gamma_{1}\right]^{-1}(\boldsymbol{\sigma} \cdot \boldsymbol{p}) \psi_{1}=\epsilon \psi_{1} .
$$

For low energies, $\epsilon \ll \gamma_{1}$, we get $\left[\epsilon-\sigma_{x} \gamma_{1}\right]^{-1} \approx-\frac{\sigma_{x}}{\gamma_{1}}$ and

$$
\left\{-\frac{v^{2}}{\gamma_{1}}\left[\sigma_{x}\left(p_{x}^{2}-p_{y}^{2}\right)+2 \sigma_{y} p_{x} p_{y}\right]+\xi v_{3}\left(\sigma_{x} p_{x}-\sigma_{y} p_{y}\right)\right\} \psi_{1} \equiv \hat{\boldsymbol{H}}_{\text {eff }} \psi_{1}=\epsilon \psi_{1}
$$

The above effective Hamiltonian describes the electronic dispersion for energies close to the neutrality point while neglecting the split bands. The basis of $\hat{\boldsymbol{H}}_{\text {eff }}$ is $\left(\phi_{+, A 1}, \phi_{+, B 2}\right)^{T}$ at the $K_{+}$and $\left(\phi_{-, B 2}, \phi_{-, A 1}\right)^{T}$ at the $K_{-}$valley. The resulting electronic dispersion,

$$
\epsilon_{\xi}= \pm\left[\left(\frac{v^{2}}{\gamma_{1}}\right)^{2} p^{4}+v_{3}^{2} p^{2}-\frac{2 \xi v^{2} v_{3}}{\gamma_{1}} p^{3} \cos 3 \varphi\right]^{\frac{1}{2}},
$$

where $p^{2}=p_{x}^{2}+p_{y}^{2}$ and $\arctan \varphi=\frac{p_{y}}{p_{x}}$, is shown in Fig. 2.5(a). The trigonally warped isoenergetic line undergoes a splitting into four pockets at the energy $\epsilon_{L T}= \pm \frac{\gamma_{1}}{4}\left(\frac{v_{3}}{v}\right)^{2} \sim 1 \mathrm{meV}$ (a so called Lifshitz transition [100]), see Fig. 2.5(b). However, characteristic values for this transition energy $\epsilon_{L T}$ and momentum $p_{L T} \sim \frac{\gamma_{1} v_{3}}{v^{2}}$ are below the resolution of any of spectroscopies considered in the following chapters. Hence, the only importance of $v_{3}$ for our considerations is 
(a)

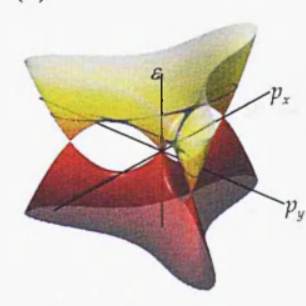

(b)



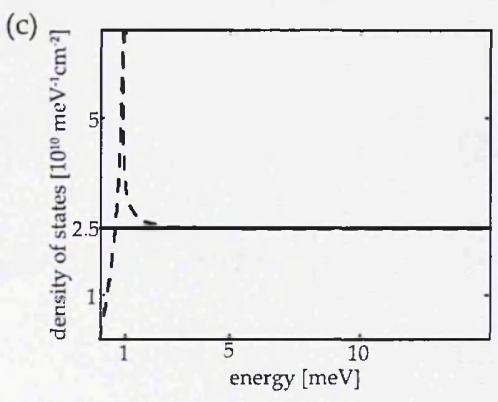

Figure 2.5: (a) The electronic dispersion of bilayer graphene at very low energies $\epsilon \sim 1 \mathrm{meV}$ around the valley $K_{+}$. The blue contour represents the isoenergetic line at the Lifshitz transition energy $\epsilon_{L T}= \pm \frac{\gamma_{1}}{4}\left(\frac{v_{3}}{v}\right)^{2}$. (b) The isoenergetic lines around the valley $K_{+}$for energies $\epsilon_{L T} / 2$ (green solid line), $\epsilon_{L T}$ (blue solid line) and $2 \epsilon_{L T}$ (red solid line). Also shown are the isoenergetic line at the energy $10 \mathrm{meV}$ from the neutrality point (black solid line) and its shape if the trigonal warping due to $v_{3}$ is neglected (black dashed line). (c) The density of states given by equation (2.13) for $v_{3}=0.1 v$ (dashed line) and $v_{3}=0$ (solid line). The latter corresponds to a purely parabolic bottom of the band.

its role as the main source of trigonal warping for the isoenergetic lines at low energies. For these energies, trigonal warping does not significantly affect the density of states (DOS), as shown in Fig. 2.5(c), where the comparison between the density of states of a purely parabolic band in the case of $v_{3}=0$ (black solid line) and of the electronic dispersion given by eq. (2.13) (black dashed line), is shown. The peak in the DOS corresponds to the Lifshitz transition. However, for energies $\epsilon>5 \mathrm{meV}$ the density of states already closely follows constant density of states for a parabolic band, although the isoenergetic lines may still be significantly noncircular (Fig. 2.5(b)).

The procedure applied above to obtain the low-energy description of electrons can be easily generalised to include all other terms appearing in the fourband Hamiltonian in linear approximation, eq. (2.9), as well as the on-site asymmetries from Sec. 2.2.3. We then obtain [99]

$$
\hat{\boldsymbol{H}}_{\mathrm{eff}}=\hat{\boldsymbol{H}}_{0}+\hat{\boldsymbol{h}}_{w}+\hat{\boldsymbol{h}}_{4}+\hat{\boldsymbol{h}}_{n}+\hat{\boldsymbol{h}}_{u}+\hat{\boldsymbol{h}}_{\Delta}+\hat{\boldsymbol{h}}_{A B}
$$


where

$$
\begin{aligned}
& \hat{\boldsymbol{H}}_{0}=-\frac{v^{2}}{\gamma_{1}}\left[\sigma_{x}\left(p_{x}^{2}-p_{y}^{2}\right)+2 \sigma_{y} p_{x} p_{y}\right] ; \\
& \hat{\boldsymbol{h}}_{w}=\xi v_{3}\left(\sigma_{x} p_{x}-\sigma_{y} p_{y}\right)-\eta \frac{v_{3}}{v}\left[\sigma_{x}\left(p_{x}^{2}-p_{y}^{2}\right)+2 \sigma_{y} p_{x} p_{y}\right] ; \\
& \hat{\boldsymbol{h}}_{4}=2 \frac{\gamma_{4} v^{2}}{\gamma_{0} \gamma_{1}} p^{2} \\
& \hat{\boldsymbol{h}}_{n}=-\frac{\gamma_{n} v^{2}}{\gamma_{0}^{2}} p^{2} \\
& \hat{\boldsymbol{h}}_{u}=\xi u\left(\frac{1}{2}-\frac{v^{2}}{\gamma_{1}^{2}} p^{2}\right) \sigma_{z} ; \\
& \hat{\boldsymbol{h}}_{\Delta}=\Delta\left(\frac{1}{2}-\frac{v^{2}}{\gamma_{1}^{2}} p^{2}\right) ; \\
& \hat{\boldsymbol{h}}_{A B}=\xi \frac{\Delta_{A B}}{2} \sigma_{z} .
\end{aligned}
$$




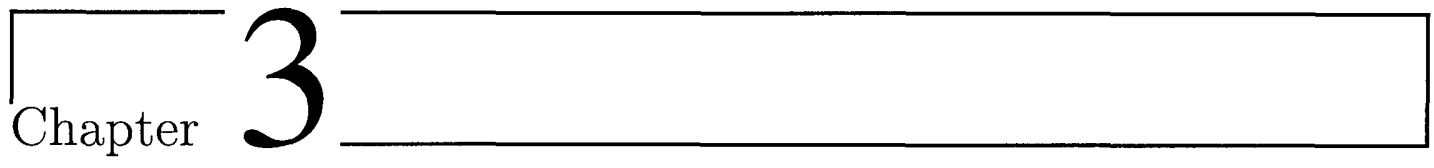

\section{Angle-Resolved Photoemission Spectroscopy}

The angle-resolved photoemission spectroscopy (ARPES) is a powerful experimental tool based on the photoelectric effect, first observed by Hertz more than 120 years ago [101] and explained by Einstein at the beginning of the previous century with the help of the then novel, idea of photons, quanta of electromagnetic radiation [102]. In the photoelectric effect, absorption of a sufficiently energetic incident photon with energy $\omega$ ejects an electron with the initial energy $\epsilon_{p}$ corresponding to the momentum $p=\hbar k$ from the sample into the vacuum. In ARPES, such ejected electrons (called photoelectrons) with kinetic energy $\epsilon_{e}$ are detected with the help of a hemispherical detectors, so that both their energy and momentum $\boldsymbol{p}_{e}$ can be identified. The modulus of the latter is given by $p_{e}=\sqrt{2 m_{e} \epsilon_{e}}\left(m_{e}\right.$ is the electron mass), while its components,

$$
\left(p_{e}\right)_{x}=\sqrt{2 m_{e} \epsilon_{e}} \cos \phi \sin \theta ;\left(p_{e}\right)_{y}=\sqrt{2 m_{e} \epsilon_{e}} \sin \phi \sin \theta ; p_{e}^{\perp}=\sqrt{2 m_{e} \epsilon_{e}} \cos \theta ;
$$

where $\phi$ and $\theta$ are the azimuthal and polar angles of detection, respectively. Knowledge of electronic states in the sample is gained with the help of two conservation laws: (I) conservation of the energy in the whole process puts a constraint on $\epsilon_{p}$, while (II) conservation of the in-plane-momentum resulting from in-plane crystallic periodicity yields some information about the electron momentum in the crystal, $\boldsymbol{p}$. For bulk materials, the angular distributions of measured photoelectrons as a function of $\epsilon_{p}$ are difficult to analyse, due to the 
lack of sufficient restrain on the out-of-plane component of $\boldsymbol{p}$. For layered or (quasi-)two-dimensional systems, however, as long as the incident radiation is monochromatic, those distributions represent direct connection to the constantenergy countours of the band structure of the material.

Because of its layered nature, graphite has been an object of extensive ARPES studies in the past [103-109]. Poor angular and energy resolutions resulted in little data concerning the $\pi$ bands in the vicinity of the $K$ points, although significant variation in the intensity from states on the same isoenergetic line has been noticed [108, 109]. Much more sophisticated equipment was available at the moment when monolayer graphene was isolated. Hence, ARPES was the method of choice for numerous investigations of the electronic band structure of two-dimensional graphene systems. It has been used to examine the epitaxial growth and confirm the graphene-like linear dispersion relation for electrons in carbon layers on $\mathrm{SiC}[33,34], \mathrm{Ni}$ [35-38], Ir [39] or $\mathrm{Ru}[42]$. At the same time, high resolution allowed detailed examination of the deviations from this linearity $[56,57,110,111]$, which stimulated numerous theoretical considerations of the band renormalisation due to many-body interactions [112-117] and some controversy on the possibility of the substrate-induced sublattice symmetry breaking in the monolayer grown on $\operatorname{SiC}[57,118-120]$. ARPES has been used to show for the first time, the appearance of the electric-field-induced gap in the spectrum of bilayer graphene [55]. In the same work, the magnitude of the interlayer coupling $\gamma_{1}$ has been extracted. It has been further employed to investigate the effects of molecular doping of monolayer and bilayer graphene $[121,122]$. The ARPES studies have been then extended to tri- and fourlayer graphene systems [123]. Review of the photoemission studies of graphene systems grown on $\mathrm{SiC}$ can be found in $[22,26]$.

In this chapter, we aim to describe the angular distribution of the angleresolved photoemission spectroscopy intensity patterns for bilayer graphene. 
We first introduce a simple theoretical model of the photoemission process, based on the idea of multiple-source interference of electronic Bloch waves. More detailed reviews of the theoretical background of ARPES can be found, for example, in $[124,125]$. We then use our model to obtain low-energy angular distributions of ARPES intensity for monolayer graphene and demonstrate that they are a manifestation of what has been recently branded as electronic chirality and what is common to all graphene-layered systems, including graphite [109, 126]. Afterwards, we show that for bilayer graphene specifically, the anisotropy of the constant-energy maps may be used to extract information about the magnitude and sign of interlayer coupling parameters and about symmetry breaking inflicted on a bilayer by the underlying substrate.

\subsection{ARPES as quantum Young's experiment}

We consider here the following photoemission process: an incoming photon with energy $\omega>W$, where $W$ is the work function of the material, is absorbed by an electron in the momentum state $k$ with energy $\epsilon_{k}$. This electron receives thus, enough energy to overcome the energetic barrier, described by the work function $W$, and leave the material. It is then detected as having energy $\epsilon_{e}$ and momentum $\boldsymbol{p}_{e}$, connected by Eq. (3.1). The energy conservation in the whole process can be expressed as

$$
\omega+\epsilon_{k}=W+\epsilon_{e}
$$

We treat the electron leaving the material as a simple case of a wave passing a potential step. It follows then from the periodicity of the sample in the plane, that the in-plane component of the momentum is conserved,

$$
\hbar(\boldsymbol{k}+\boldsymbol{G})=\boldsymbol{p}_{e}^{\|}
$$


where $\boldsymbol{p}=\hbar \boldsymbol{k}, \boldsymbol{G}=m_{1} \boldsymbol{b}_{1}+m_{2} \boldsymbol{b}_{2}$ is the reciprocal lattice vector and we choose the $z$ axis to correspond to the direction perpendicular to the plane of our sample and $\left(p_{e}^{\|}\right)^{2}=\sqrt{\boldsymbol{p}_{e}^{2}-\left(\boldsymbol{p}_{e}^{\perp}\right)^{2}} ; \boldsymbol{p}_{e}^{\perp}=\left(0,0, p_{e}^{\perp}\right)$.

The ARPES intensity is proportional to the modulus square of the transition amplitude between the initial and final states of system under the perturbation caused by incoming radiation. We treat the latter with the perturbative Hamiltonian

$$
\hat{\boldsymbol{H}}_{\mathrm{int}}=-\frac{e}{2 m_{e}}(\boldsymbol{A} \cdot \hat{\boldsymbol{p}}+\hat{\boldsymbol{p}} \cdot \boldsymbol{A})=-\frac{e}{m_{e}} \boldsymbol{A} \cdot \hat{\boldsymbol{p}},
$$

where $\boldsymbol{A}$ is the electromagnetic vector potential of the incoming radiation and $\hat{p}$ is the electron momentum operator. We neglect many-body interactions and as the initial state take the single-electron Bloch wave state in the general form, Eq. (2.2),

$$
\Psi(r)=\sum_{i} C_{i} \phi_{k, i}(r),
$$

where we have for now dropped the index $j$ and consider a single band. As for the final state of the electron, $\Psi_{e}(r)$, we approximate it with a plane wave,

$$
\Psi_{e}(r) \propto \exp \left(\frac{i}{\hbar} p_{e} \cdot \boldsymbol{r}\right)
$$

We are interested in the angular distribution of ARPES probing the low-energy electronic states in the vicinity of the valleys, not the absolute value of the intensity. Hence, neglecting prefactors not contributing to the angle-dependence, we express the ARPES intensity from electron states in a given band as

$$
I \propto\left|\left\langle e^{\frac{i}{\hbar} p_{e} \cdot \boldsymbol{r}}|\boldsymbol{A} \cdot \boldsymbol{p}| \sum_{i, \boldsymbol{R}_{0}} C_{i} e^{i \boldsymbol{k} \cdot\left(\boldsymbol{R}_{0}+\boldsymbol{R}_{i}\right)} \varphi\left(\boldsymbol{r}-\boldsymbol{R}_{0}-\boldsymbol{R}_{i}\right)\right\rangle\right|^{2} \delta\left(\epsilon_{e}+W-\epsilon_{p}-\omega\right) .
$$

At the same time, we expect the patterns to reflect the shape of the isoenergetic lines around the valleys. The radius of the area in the reciprocal space around a single valley important for our considerations is less than ten per cent of the 
$\Gamma-K$ distance. Also, the shapes of the patterns for a specific energy should resemble trigonally warped circles with all important angle-dependent features contained within a narrow ring in the reciprocal (or momentum) space. We assume, that for this narrow range of momenta, the result of the perturbation operator $\boldsymbol{A} \cdot \hat{\boldsymbol{p}}$ acting on the initial state is a smooth, slowly varying function of momentum and approximate it with an irrelevant constant. This assumption is justified, as the energy of incoming photons used in experiments is of the order of $50-150 \mathrm{eV}[22,34,42,55,57,118,123]$ translating into the lower bound on the photon's wavelength $\lambda \gtrsim 5 \mathrm{~nm}$, corresponding to about twenty lattice constants. This means that the incoming electromagnetic wave does not distinguish details of the electron Bloch state (for example the structure of the atomic $2 p_{z}$ orbital). Therefore, the intensity $I$ can be related to

$$
I \propto\left|\sum_{i} C_{i} e^{\frac{i}{\hbar}\left(p-p_{e}\right) \cdot \boldsymbol{R}_{i}} \sum_{\boldsymbol{R}_{0}} e^{\frac{i}{\hbar}\left(p-p_{e}\right) \cdot \boldsymbol{R}_{0}}\left\langle e^{\frac{i}{\hbar} p_{e} \cdot r^{\prime}} \mid \varphi\left(r^{\prime}\right)\right\rangle\right|^{2} \delta\left(\epsilon_{e}+W-\epsilon_{p}-\omega\right),
$$

where we introduced new position vector $\boldsymbol{r}^{\prime}=\boldsymbol{r}-\boldsymbol{R}_{0}-\boldsymbol{R}_{i}$. Sum over the two-dimensional lattice vectors $R_{0}$ of the phase factors leads to the Dirac delta expressing conservation of the in-plane momentum, Eq. 3.3. The integral over $\boldsymbol{r}^{\prime}$ is the Fourier image of the atomic $2 p_{z}$ orbital $\varphi\left(\boldsymbol{r}^{\prime}\right)$, which we denote by $\varphi\left(\boldsymbol{p}_{e}\right)$. We obtain

$$
I \propto\left|\varphi\left(\boldsymbol{p}_{e}\right)\right|^{2}\left|\sum_{i} C_{i} e^{-i \boldsymbol{G} \cdot \boldsymbol{R}_{i}} e^{-\frac{i}{\hbar} p_{e}^{\perp} \cdot \boldsymbol{R}_{i}}\right|^{2} \delta\left(\epsilon_{e}+W-\epsilon_{\boldsymbol{p}}-\omega\right) .
$$

Let us now consider the Fourier transform $\varphi\left(p_{e}\right)$ as a function written in spherical coordinates, $\varphi\left(\boldsymbol{p}_{e}\right) \equiv \varphi\left(p_{e}, \phi, \theta\right)$. Just like the $2 p_{z}$ orbital in the real space, its Fourier transform $\varphi\left(\boldsymbol{p}_{e}\right)$ has rotational symmetry in the $p_{x}-p_{y}$ plane and does not depend on the azimuthal angle $\phi$. For a given energy of the incoming photons $\omega$ and material specific work function $W$, to resolve the constantenergy maps of the ARPES intensity for energy $\epsilon_{p}$, one only needs to look at 
photoelectrons with well specified energy $\epsilon_{e}=\omega-W+\epsilon_{p}$ and thus the modulus of the momentum $p_{e}$. Finally, as we concentrate on a small area in the momentum space around the valleys, the resulting change in the polar angle $\theta$ is going to be small. For the momentum states under consideration, $\varphi\left(\boldsymbol{p}_{e}\right)$ is almost a constant. Therefore, we neglect the prefactor $\left|\varphi\left(p_{e}\right)\right|^{2}$. We will also first treat the lattice as strictly two-dimensional, which means that $e^{-\frac{i}{\hbar} p_{e}^{\perp} \cdot R_{i}}=1$. We end with the expression

$$
I \propto\left|\sum_{i} C_{i} e^{-i G \cdot R_{i}}\right|^{2} \delta\left(\epsilon_{e}+W-\epsilon_{k}-\omega\right) .
$$

In the formula above, the ARPES intensity pattern arises as a result of the interference of the photoelectron waves originating from all atomic sites within the unit cell. The contribution of the $i$-th site is given by the amplitude on that $i$-th site of the electronic Bloch wave function corresponding to the initial state with energy $\epsilon_{k}$. These amplitudes are simply the coefficients of the eigenstates for the tight-binding Hamiltonians, as is evident from Eq. (2.2) and (2.4). The exponential factors $\exp \left(-i \boldsymbol{G} \cdot \boldsymbol{R}_{i}\right)$ take into account the in-plane difference in optical paths between electron waves originating on different sites and travelling towards the detector. Hence, in this simplified description, ARPES patterns correspond to a electron-wave version of quantum Young's double-slit experiment [127]. The number of sources equals in this case the number of atoms in the unit cell. Conservation of the in-plane momentum maps directly the ARPES pattern of the photoelectron momentum to the constant-energy cuts through the band structure of our material, while conservation of energy defines the total wavevector $(k+G)$ observable in the experiment. The greater the energy of the incoming photons, the more Brillouin zones can be resolved. Finally, we do not model here dynamical effects that lead to energy broadening [56, 112-117] but introduce a Lorentzian $\delta(\cdots) \approx \pi^{-1} \Gamma /\left[(\cdots)^{2}+\Gamma^{2}\right]$ in the figures with the parameter $\Gamma$ representing finite energy broadening. 


\subsection{Monolayer Graphene}

To derive the angular distributions of the ARPES intensity for monolayer graphene, we use the tight-binding Hamiltonian, which can be obtained in a procedure very similar to that presented for bilayer graphene in Chapter 2. Considering only one layer of hexagonally arranged carbon atoms (for example the bottom one in Fig. 2.1), one ends with the same unit vectors, unit cell and Brillouin zone. However, the unit cell now contains only two atoms, $A$ and $B$, with their positions within the unit cell given as $\boldsymbol{R}_{A}=-\boldsymbol{d}_{1}$ and $\boldsymbol{R}_{B}=\mathbf{0}$, respectively. Within the linear approximation, the Hamiltonian of monolayer graphehe in the basis $\left(\phi_{+, A}, \phi_{+, B}\right)^{T}$ or $\left(\phi_{-, B}, \phi_{-, A}\right)^{T}$ depending on the valley, is $[2,78,84]$

$$
\hat{\boldsymbol{H}}_{11}=\xi v\left(\begin{array}{cc}
0 & \hat{\pi}^{\dagger} \\
\hat{\boldsymbol{\pi}} & 0
\end{array}\right) .
$$

From this follow the energy eigenvalue $\epsilon_{p}$ and corresponding eigenstates $\psi_{p}$,

$$
\epsilon_{p}=s v p, \quad \psi_{p}=\frac{1}{\sqrt{2}}\left(\begin{array}{c}
e^{-i \frac{\varphi}{2}} \\
\xi s e^{i \frac{\varphi}{2}}
\end{array}\right),
$$

where $\varphi=\arctan \frac{p_{y}}{p_{x}}$ and $s= \pm 1$ denotes the conduction $(s=1)$ or the valence $(s=-1)$ band. Both the conduction and valence bands have linear dispersion. They touch each other exactly in the center of the valley at the energy $\epsilon_{D}$ usually taken as zero of the energy scale. This characteristic feature leads to the $K$ points often being called the Dirac points [13, 14, 25]. With the use of Eq. (3.9), the angular distribution of ARPES for monoalyer graphene is related to

$$
I \propto \frac{1}{2}\left|e^{-i \frac{\varphi}{2}} e^{-i \frac{\xi}{2} G \cdot d_{1}}+\xi S e^{i \frac{\varphi}{2}} e^{i \frac{\xi}{2} G \cdot d_{1}}\right|^{2}=1+\xi S \cos \left[\varphi-\xi \frac{2 \pi}{3}\left(m_{2}-m_{1}\right)\right] .
$$


The two real numbers, $m_{1}$ and $m_{2}$, define which Brillouin zone the ARPES spectrum is described for. However, as the vicinity of any valley belongs formally to one of the three neighbouring Brillouin zones (due to the $K$ point being a corner of a hexagon), for further simplicity of our discussion, we choose different unit cell in the reciprocal space, that is, a rhombus centred on the $\Gamma$ point, as shown in Fig. 2.1(b). This choice does not influence any of the formulae in this chapter, yet simplifies the problem of choosing the numbers $m_{1}$ and $m_{2}$ as the vicinity of any $K$ point is now contained in a single unit cell. For the two representative valleys $K_{+}$and $K_{-}$, equation (3.12) can now be put in the simple form

$$
I \propto\left\{\begin{array}{ll}
\cos ^{2}\left[\frac{\varphi}{2}-\xi \frac{\pi}{3}\left(m_{2}-m_{1}\right)\right] & \text { for } \xi S=1 \\
\sin ^{2}\left[\frac{\varphi}{2}-\xi \frac{\pi}{3}\left(m_{2}-m_{1}\right)\right] & \text { for } \xi s=-1
\end{array} .\right.
$$

We see that as the path around the valley is traversed and the angle $\varphi$ changes, one peak in the intensity is observed, at the angle $\left[\varphi=\xi \frac{2 \pi}{3}\left(m_{2}-m_{1}\right) \bmod 2 \pi\right]$ if $\xi_{S}=1$ or $\left[\varphi=\pi+\xi \frac{2 \pi}{3}\left(m_{2}-m_{1}\right) \bmod 2 \pi\right]$ if $\xi s=-1$. The ARPES patterns probing states at the same energy $\epsilon_{p}$ differ between the valleys. However, the angular distribution around the valley $K_{+}$for the valence band is the same as the angular distribution around the valley $K_{-}$for the conduction band. The same is true for patterns around $K_{+}$for the conduction band and $K_{-}$for the valence band.

Numerically calculated ARPES patterns for monolayer graphene within the general tight-binding model, that is the band structure described as

$$
\left(\begin{array}{cc}
0 & -\gamma_{0} f(\boldsymbol{k}) \\
-\gamma_{0} f^{*}(\boldsymbol{k}) & 0
\end{array}\right) \psi(\boldsymbol{p})=\epsilon_{p}\left(\begin{array}{cc}
1 & s_{0} f(\boldsymbol{k}) \\
s_{0} f^{*}(\boldsymbol{k}) & 1
\end{array}\right) \psi(\boldsymbol{p}),
$$

are shown in Fig. 3.1. Although due to additional terms in the electronic momentum $\boldsymbol{p}$ contained in the geometrical factor $f$, the eigenstate can no longer be written down in a simple form containing the azimuthal angle $\varphi$, the above 

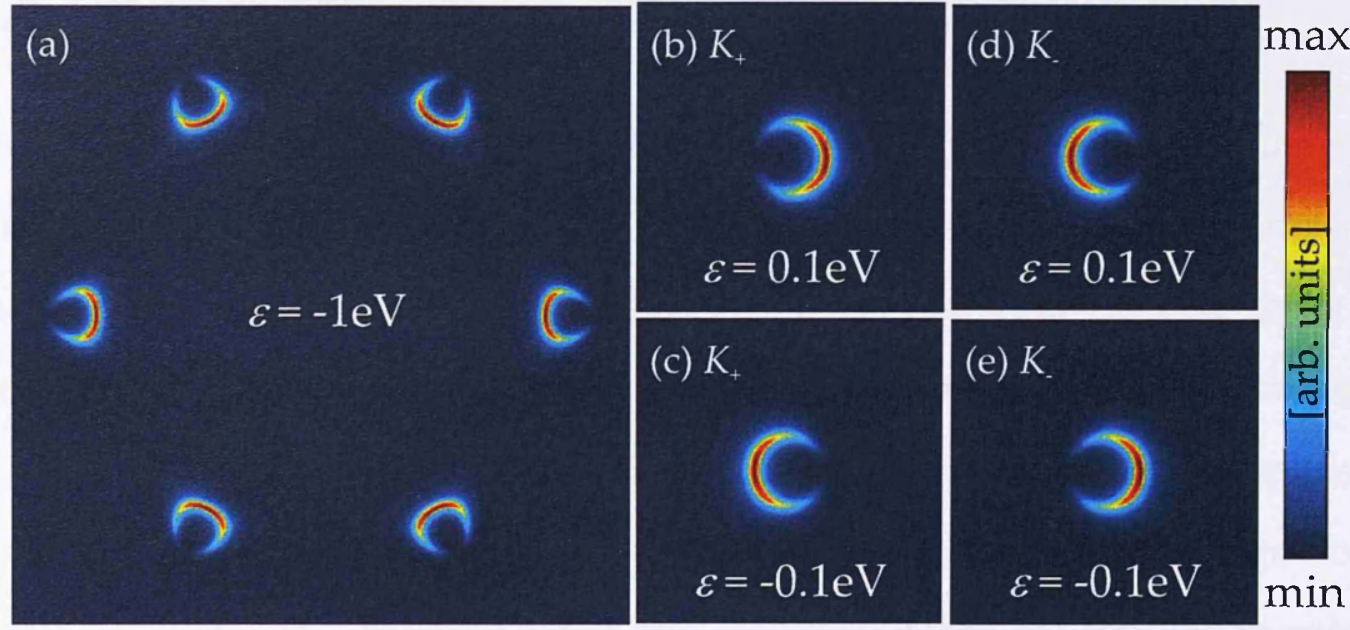

Figure 3.1: The constant-energy ARPES maps of monolayer graphene obtained within the full tight-binding model, Eq. (3.14), for (a) whole Brillouin zone; (b) and (c) valley $K_{+} ;(d)$ and (e) valley $K_{-}$. Energy corresponding to each map is given with respect to the Dirac point. The length of the side of the map in the reciprocal space is $\frac{10 \pi}{3 a}$ and $\frac{8 \pi}{75 a}$ for (a) and (b)-(e), respectively. Numerical values of the parameters used: $\gamma_{0}=3 \mathrm{eV}, s_{0}=0.129, a=2.46 \AA$.

conclusions still hold. As a particular valley is circled round, the ARPES intensity exhibits one peak (intensity is at maximum) and one dip (intensity is zero). Due to those higher terms in $p$, for energies far from the Dirac point (Fig. 3.1(a)) pattern around each valley is trigonally warped. However, for energies close to the Dirac point (Fig. 3.1(b)-(e)), the isoenergetic lines are circular and the electron-hole asymmetry is negligible. Thus, the pattern around the valley $K_{+}\left(K_{-}\right)$for the valence band is the same as around the valley $K_{-}\left(K_{+}\right)$for the conduction band.

The peculiar behaviour of the ARPES intensity as a function of the azimuthal angle $\varphi$ around the valley $K_{\xi}$ has been noticed before for bulk graphite [109]. As shown, its origins lie purely in the hexagonal symmetry of graphene. This symmetry gives rise to a specific phase relation between components of the electron wave on the two sublattices and the electron's momentum. In recent literature, this is often reffered to as chirality of electrons in graphene $[13,14$, $25,96]$. The angle-resolved photoemission provides thus a direct observation of this phenomenon. 


\subsection{Bilayer Graphene}

As explained in the previous section, the main features in the angular distribution of the ARPES intensity for graphene have been in the past observed for graphite. As graphene and graphite are conceptually the extreme cases of hexagonally layered carbon system, one might expect no significant differences in the ARPES spectra for systems in between, that is also for bilayer graphene. However, the low-energy electronic spectrum undergoes a drastic change from linear to quadratic dispersion when two graphene layers are coupled together to form a bilayer. The question is therefore, whether this change leads to new features in the angular distribution of the ARPES intensity.

\subsubsection{Low-energy spectrum: contribution of the two degenerate bands only}

As we are concerned with the low-energy electronic properties, we start with the effective two-band approximation, Eq. (2.12). For simplicity of the arguments that follow, we neglect the $v_{3}$ terms and write the eigenproblem

$$
-\frac{v^{2}}{\gamma_{1}}\left(\begin{array}{cc}
0 & \left(\hat{\pi}^{\dagger}\right)^{2} \\
\hat{\pi}^{2} & 0
\end{array}\right) \psi=\epsilon \psi
$$

We write the resulting spectrum and electronic eigenstates as follows:

$$
\epsilon_{p}=s \frac{v^{2}}{\gamma_{1}} p^{2} ; \quad \psi=\frac{1}{\sqrt{2}}\left(\begin{array}{c}
e^{-i \varphi} \\
-s e^{i \varphi}
\end{array}\right)
$$

where $s$, as before, swaps between the conduction $(s=1)$ and valence $(s=-1)$ bands and $\varphi=\arctan \frac{p_{y}}{p_{x}}$. The eigenstates above are very similar to those in the case of the monolayer, Eq. (3.11), although the prefactor $\frac{1}{2}$ in front of the angle 


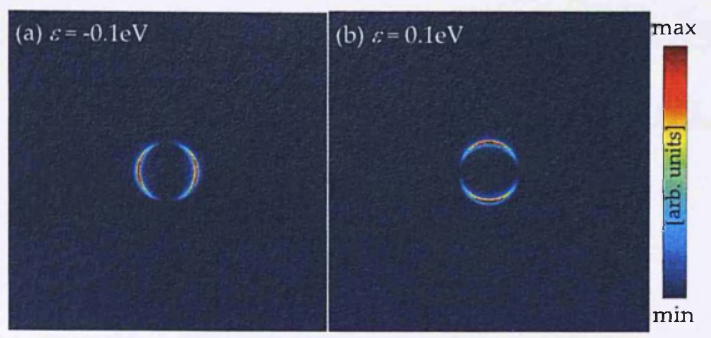

Figure 3.2: The constant-energy ARPES maps of bilayer graphene obtained within the low-energy two-band approximation, Eq. (3.14), for energies (a) $\epsilon=-0.1 \mathrm{eV}$, (b) $\epsilon=0.1 \mathrm{eV}$ with respect to the neutrality point. The length of the side of the map in the reciprocal space is $\frac{4 \pi}{15 a}$, numerical values of the parameters used: $v=0.97 \mathrm{~m} / \mathrm{s}, \gamma_{1}=0.4 \mathrm{eV}$.

$\varphi$ and valley index $\xi$ are missing. From Eq. (3.9), the ARPES intensity is

$$
I \propto\left\{\begin{array}{ll}
\cos ^{2}\left[\varphi+\xi \frac{2 \pi}{3}\left(m_{2}-m_{1}\right)\right] & \text { for } s=-1 \\
\sin ^{2}\left[\varphi+\xi \frac{2 \pi}{3}\left(m_{2}-m_{1}\right)\right] & \text { for } s=1
\end{array} .\right.
$$

Comparison between (3.17) and (3.13) shows that for bilayer graphene the angular distribution of the ARPES intensity around a valley should depends on twice the characteristic angle obtained for monolayer, $\left[\frac{\varphi}{2}-\frac{\pi}{3}\left(m_{2}-m_{1}\right)\right]$. Therefore, two symmetric peaks and two dips in the intensity are expected at low energies as the path around the valley is traversed. That is shown in the Fig. 3.2. No difference (except possibly trigonal warping effects) should occur when changing the valley from $K_{+}$to $K_{-}$. Also, a rotation by $\pi / 2$ should occur when swapping the band.

\subsubsection{Contribution from the split bands}

In the previous section we described the ARPES spectra in the low-energy limit. At the same time, the ARPES pattern should evolve so that at higher energies it resembles that of monolayer graphene or graphite, Fig. 3.1(a), with one peak in the intensity. Such a regime can not be described with the low energy approximation. We move therefore to the four-band Hamiltonian, which not only enables us better comparison to experimental data, but also, as it turns out, 
adds to the low-energy description from the previous section [58]. Again, for the sake of the argument, we start with the minimum model describing all four bands around the valley $K_{\xi}$, that is,

$$
\left(\begin{array}{cccc}
0 & 0 & 0 & \xi v \pi^{+} \\
0 & 0 & \xi v \pi & 0 \\
0 & \xi v \pi^{\dagger} & 0 & \gamma_{1} \\
\xi v \pi & 0 & \gamma_{1} & 0
\end{array}\right) \psi=\epsilon \psi
$$

Once again, we require the knowledge of the eigenstates and the energies,

$$
\epsilon_{s b}=s \frac{1}{2}\left(\sqrt{\gamma_{1}^{2}+4 v^{2} p^{2}}+b \gamma_{1}\right), \quad \psi_{s b}=\frac{1}{\sqrt{2} \sqrt{1+\left(\frac{\mid \varepsilon_{s b b}}{v p}\right)^{2}}}\left(\begin{array}{c}
e^{-i \varphi} \\
s b e^{i \varphi} \\
\xi b \frac{\left.\right|_{s b l} \mid}{v p} \\
\xi s \frac{||_{s b} \mid}{v p}
\end{array}\right),
$$

where $b= \pm 1$ distinguishes between the split and the low-energy bands. Note that what $b(1$ or -1$)$ corresponds to which set of bands depends on the sign of $\gamma_{1}$. This does not matter for the band structure (does not change the form of the eigenvalues, only reorders them), but does influence the form of the eigenstate corresponding to each band. The angular distribution of the ARPES intensity is proportional to

$$
\begin{aligned}
I & \propto\left(1+s b \cos \left[2 \varphi+\xi \frac{4 \pi}{3}\left(m_{2}-m_{1}\right)\right]\right. \\
& \left.+2 \delta_{s b}\left(\frac{\left|\epsilon_{b s}\right|}{v p}\right)\left\{\frac{\left|\epsilon_{b s}\right|}{v p}+(b+s) \cos \left[\varphi+\xi \frac{2 \pi}{3}\left(m_{2}-m_{1}\right)\right]\right\}\right) .
\end{aligned}
$$

The first two terms correspond to the low energy limit discussed in the previous section. The last term is then a correction, which vanishes for $s b=-1$. In this case one is left with the pattern of two symmetric peaks, as in Fig. 3.2. However, this happens for only one of the two low-energy bands (and one high-energy band). 
For the other, $s b=1$, the correction does not vanish and contributes towards the intensity. To estimate its importance, we consider valleys $\boldsymbol{K}_{ \pm}=\left( \pm \frac{4 \pi}{3 a}, 0\right)$ in the first Brillouin zone $\left(m_{1}, m_{2}=0\right)$. Also, for the low energy bands, $|\epsilon| \approx \frac{v^{2}}{\gamma_{1}} p^{2}$. Then,

$$
I \propto\left(1+\cos (2 \varphi)+4 s \sqrt{\frac{\left|\epsilon_{s b}\right|}{\gamma_{1}}} \cos \varphi+2 \frac{\left|\epsilon_{s b}\right|}{\gamma_{1}}\right)
$$

This function has two maxima for $\varphi \in(0,2 \pi)$, at $\varphi=0$ and $\varphi=\pi$. The ratio of their intensities is

$$
\frac{I(\varphi=0)}{I(\varphi=\pi)}=\left(\frac{1+s \sqrt{\frac{|\epsilon|}{\gamma_{1}}}}{1-s \sqrt{\frac{|\epsilon|}{\gamma_{1}}}}\right)^{2} .
$$

For the energy $\left|\epsilon_{s b}\right|=0.1 \mathrm{eV}$ from the neutrality point, the above ratio yields $\frac{25}{9}$ for $s=1$ or $\frac{9}{25}$ for $s=-1$. Hence, one of the peaks is more than twice higher than the other. That strong asymmetry is obtained for energies of the order of a quarter of $\gamma_{1}$, which are usually considered to be in all instances well described by the two-band approximation.

To summarise, according to Eq. (3.20), we expect the symmetric two-peak pattern to appear for two of the bands. For $\gamma_{1}>0$, these are the valence split band $(s=-1, b=1)$ and the conduction low-energy band $(s=1, b=-1)$. For the other two bands, we expect one of the peaks to go darker as we increase the distance from the neutrality point. From the band structure considerations, Eq. (2.9), we also expect the pattern to be strongly trigonally warped at low energies due to the coupling $\gamma_{3}$. This low-energy warping does not occur in the monolayer case.

The constant-energy maps of the ARPES intensity, calculated within the full four-band model, Eq. (2.4) and (2.6), are presented in Fig. 3.3 for the whole Brillouin zone, (a), and valley $K_{+}$(b)-(g). As anticipated, for the energy far from the neutrality point, Fig. 3.3(a), the ARPES spectrum look similar to that of the monolayer, 3.1(a) and graphite $[109,126]$. Figures 3.3(b)-(g) show the evolution 

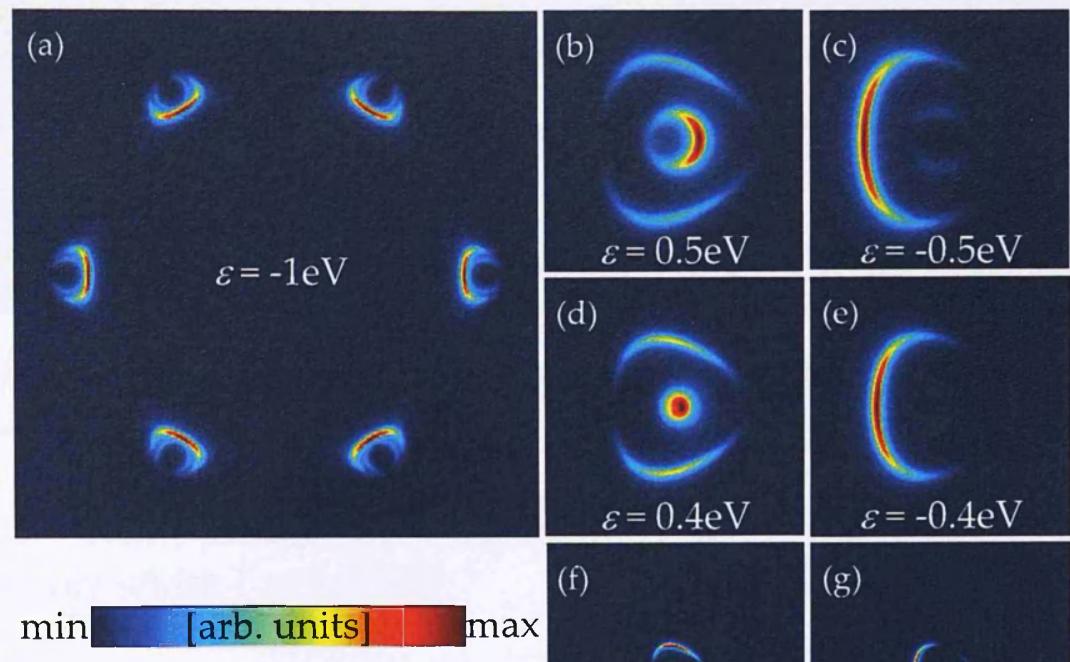

(f)

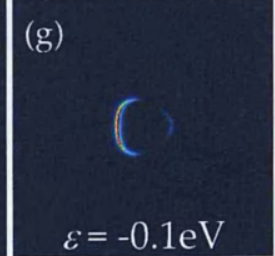

Figure 3.3: The constant-energy ARPES maps of bilayer graphene obtained within the four-band tight-binding model, Eq. (2.4) and (2.6). All energies are given with respect to the neutrality point. The length of the side of the map in the reciprocal space is (a) $\frac{16 \pi}{5 a}$, (b)-(g) $\frac{4 \pi}{15 a}$. Numerical values of the parameters used: $\gamma_{0}=3 \mathrm{eV}, a=2.46 \AA$ (resulting $\left.v \approx 0.97 \mathrm{~m} / \mathrm{s}\right), \gamma_{1}=0.4 \mathrm{eV}, \gamma_{3}=0.2 \mathrm{eV}, s_{0}=0.129$, and the energy width $\Gamma$ is one sixth of the corresponding energy.

of the ARPES pattern as the energy changes from $0.5 \mathrm{eV}$ to $-0.5 \mathrm{eV}$. At energies greater than the interlayer coupling, $\epsilon>\gamma_{1},(b)$ and $(c)$, there are two ringlike patterns, each corresponding to photoemission from states in two bands, whereas, for low-energies, $\epsilon<\gamma_{1}$, (d)-(g), there is a single ring corresponding to emission from the degenerate band only. The dot in the center of 3.3(d) corresponds to the photoemission from the bottom of the split band due to finite energy width $\Gamma$. Disappearance of this dot provides an estimate for the magnitude of the parameter $\gamma_{1}$ [55]. It is not visible on Fig. 3.3(e), because for this band $s b=-1$. As mentioned before, the sign of $\gamma_{1}$ determines for which set of bands the pattern of two equally bright peaks should be observe. In agreement with the conclusions from Eq. 3.20, in the Fig. 3.3(b)-(g) these symmetric peaks are observed for the low-energy conduction and high-energy valence bands as $\gamma_{1}$ was taken to be positive. Negative sign of $\gamma_{1}$ would lead to equally bright peaks appearing in the high-energy conduction and low-energy valence bands. 

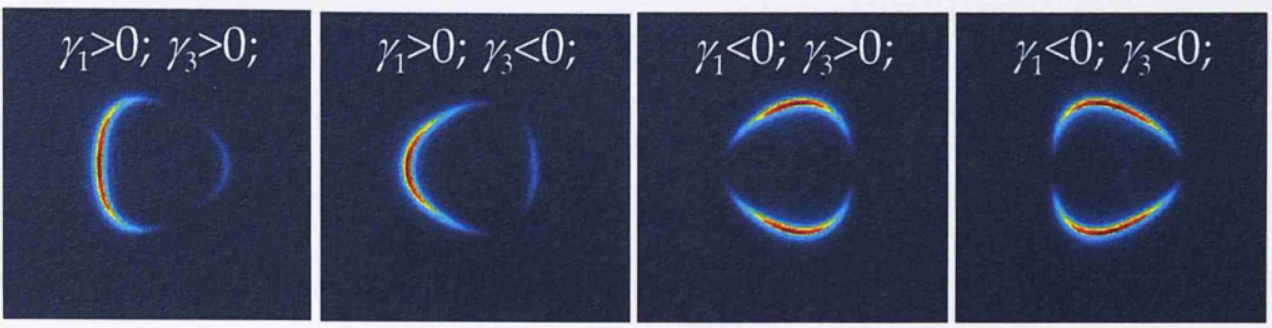

Figure 3.4: The influence of the signs of $\gamma_{1}$ and $\gamma_{3}$ on the ARPES spectra for the energy $\epsilon=-0.1 \mathrm{eV}$ with respect to the neutrality point. The length of the side of the map in the reciprocal space is $\frac{4 \pi}{30 a}$. Numerical values of the parameters used: $\gamma_{0}=3 \mathrm{eV}, a=2.46 \AA$ (resulting $v \approx 0.97 \mathrm{~m} / \mathrm{s}$ ), $\left|\gamma_{1}\right|=0.4 \mathrm{eV},\left|\gamma_{3}\right|=0.2 \mathrm{eV}, s_{0}=0.129$, and the energy width $\Gamma=0.1 / 6 \mathrm{eV}$.

Hence, probing the electron wave function via ARPES may reveal not only the magnitude of the coupling but also its sign. We emphasize that this sign is not important when the tight-binding model is used only to obtain the band structure and comparisons to experimentally obtained electronic dispersions relate only the band splittings which are always positive. Also, notice the second peak evolving in the pattern originating from the low-energy valence band at $\varphi=0$ as the energy shifts closer to the neutrality point. It is not visible at all at the energy $\epsilon=-0.5 \mathrm{eV}$, but is quite clear for $\epsilon=-0.1 \mathrm{eV}$, where, in agreement to the above discussion, it is less than half of the dominant peak around $\varphi=\pi$.

As mentioned above, the sign of the $\gamma_{1}$ coupling may be extracted from the ARPES spectra. We now show that knowledge of this sign allows for the determination of the sign of the trigonal warping parameter, $\gamma_{3}$. Let us recall the low energy dispersion around the valley $K_{\xi}$ as described by the two-band approximation, Eq. (2.13),

$$
\epsilon_{\xi}=s\left[\left(\frac{v^{2}}{\gamma_{1}}\right)^{2} p^{4}+v_{3}^{2} p^{2}-\frac{2 \xi v^{2} v_{3}}{\gamma_{1}} p^{3} \cos 3 \varphi\right]^{\frac{1}{2}} .
$$

This expression illustrates that the angular dependent factor, which produces trigonal warping, depends on the sign of the ratio $\gamma_{3} / \gamma_{1}$. Once one of the signs is set, the other follows from investigation of the shape of the ARPES patterns 
at low energies. This is presented in Fig. 3.4, where the comparison between patterns at the energy $-0.5 \mathrm{eV}$ for different signs of $\gamma_{1}$ and $\gamma_{3}$ is shown. For $\operatorname{sgn} \gamma_{1}=\operatorname{sgn} \gamma_{3}$, the trigonal warping due to $\gamma_{3}$ deforms the isoenergetic lines in the same fashion as the high-energy trigonal warping due to higher than linear terms in the electronic momentum in the factor $f(k)$ [Fig. 3.4(a) and (d); compare to Fig. 3.3(a)]. However, for $\operatorname{sgn} \gamma_{1}=-\operatorname{sgn} \gamma_{3}$, the low-energy warping counteracts the effects of the high-energy warping [Fig. 3.4(b) and (c)]. Once the sign of $\gamma_{1}$ has been established, the direction of the low-energy warping along the $p_{x}$ axis [compare Fig. 3.4(a) and (b) or (c) and (d)] determines the sign of $\gamma_{3}$.

\subsubsection{Influence of the symmetry breaking parameters on the ARPES spectra}

The angular distributions of the ARPES intensity are also sensitive to the symmetry breaking parameters $u, \Delta$ and $\Delta_{A B}$. There are two reasons for that: (I) all of these parameters, as shown in Sec. 2.2.3, modify the band structure, thus changing the shape of the isoenergetic lines in the reciprocal space probed with ARPES; (II) these parameters also influence the amplitude $C_{i}$ of the electron Bloch wave on the atomic site $i$. The influence of the on-site asymmetries on the ARPES spectra is shown on the example of the interlayer asymmetry $u$ in Fig. 3.5. Four spectra around the $K_{+}$valley for different values of the asymmetry are presented, as well as the corresponding low-energy band structures (beneath each ARPES spectrum). The two-peak pattern is quite robust against the opening of a gap. Only when the top/bottom of the gap is near the probed energy, the pattern is distorted (last column in Fig. 3.5). The opening of the gap in the electronic spectrum due to the interlayer asymmetry $u$ has already been sucessfully observed with ARPES [55]. 




Figure 3.5: The influence of the interlayer asymmetry $u$ on the ARPES spectra for energy $\epsilon=0.1 \mathrm{eV}$ with respect to the neutrality point. The length of the side of the map in the reciprocal space is $\frac{8 \pi}{75 a}$. Shown below the spectra are corresponding electronic dispersions along the $P_{x}$ axis; the dotted lines show the position of the neutrality point (the middle of the gap) and the dashed lines show the energy the spectra are resolved for. Numerical values of the parameters used: $\gamma_{0}=3 \mathrm{eV}$, $a=2.46 \AA$ (resulting $v \approx 0.97 \mathrm{~m} / \mathrm{s}$ ), $\gamma_{1}=0.4 \mathrm{eV}, \gamma_{3}=0.2 \mathrm{eV}, s_{0}=0.129$, and the energy width $\Gamma=0.1 / 3 \mathrm{eV}$.

\subsubsection{Interference due to a finite interlayer distance}

Up to this point, we treated bilayer graphene as purely two-dimensional and disregarded the existence of the interlayer spacing between layers, $c_{0}$. Clearly, $c_{0}$ is going to influence the ARPES spectra via the phase factors describing the difference in the optical paths for photoelectron waves originating on different atomic sites, as shown in Eq. (3.8). The out-of-plane component of the momentum is

$$
p_{e}^{\perp}=\sqrt{2 m\left(\omega-W+\epsilon_{k}\right)-\hbar^{2}(k+G)^{2}},
$$

and we assume that only the photoelectrons with $p_{e}^{\perp}>0$ are detected. In order to calculate the ARPES patterns, we now need to set the values of the energy of the incoming photons $\omega$ and the material constant, work function $W$. The intensity distribution now depends on $\omega$.

Examples of the ARPES spectra for different energies of the incoming radiation have been shown in Fig. 3.6. At high-energies (Fig. 3.6(a)) the pattern does not qualitatively change. However, at low energies (Fig. 3.6(b)-(c)), when 




Figure 3.6: The influence of the interlayer spacing on the ARPES spectra for energy (a) $\epsilon=-1 \mathrm{eV}$, (b)-(c) $\epsilon=-0.2 \mathrm{eV}$ with respect to the neutrality point. The length of the side of the map in the reciprocal space is (a) $\frac{16 \pi}{5 a}$, (b)-(c) $\frac{4 \pi}{15 a}$. Numerical values of the parameters used: $\gamma_{0}=3 \mathrm{eV}, a=2.46 \AA$ (resulting $v \approx$ $0.97 \mathrm{~m} / \mathrm{s}), \gamma_{1}=0.4 \mathrm{eV}, \gamma_{3}=0.2 \mathrm{eV}, s_{0}=0.129, c_{0}=3.4 \AA, W=5 \mathrm{eV}$ and the energy width (a) $\Gamma=0.2 \mathrm{eV}$, (b)-(c) $\Gamma=0.04 \mathrm{eV}$.

the contribution due to the states in the split bands is no longer present, the dependence on $\omega$ manifests itself through a rotation of the pattern around the valley. These slightly rotated patterns resemble experimental constant-energy maps of ARPES intensity shown in the online material supporting work published in [55]. The additional rotation caused by the phase factor associated with the interlayer distance $c_{0}$ makes extracting the band structure parameters, and especially their signs, from the spectra much more difficult. However, with detailed comparison with experiment and calibration of the energy scale, it may still be possible. Then, an energy can be chosen for the incoming photons so that $\frac{p_{e}^{\perp} c_{0}}{2} \approx 0$ for photoelectrons originating from the states close to the centre of the valley. 


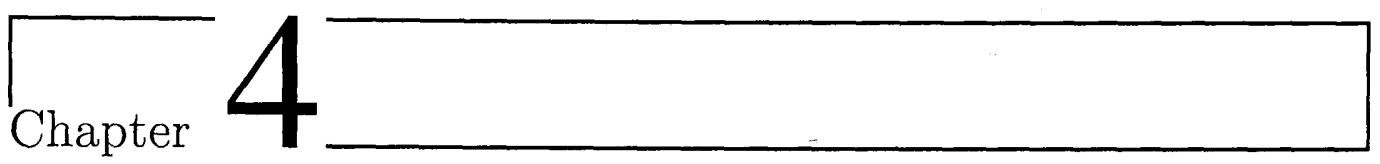

\section{Magneto-Optical Spectroscopy}

Behaviour of electrons in (quasi-)two-dimensional systems in external magnetic fields is a fascinating area of physics. Classically, the Lorentz force caused by the magnetic field curves the trajectory of a charged particle. If such a particle is constrained to move only in one plane, in a strong enough field perpendicular to that plane, the trajectory of the particle becomes a closed orbit. However, quantum mechanically, due to wave nature of matter, only some of the orbits are stable. For a two-dimensional solid in low temperatures, this results in the electronic band structure turning into a discrete spectrum of Landau levels [128]. The number of states per unit area in each Landau level (LL) (degeneracy of the $\mathrm{LL}$ ) is equal to those originally within the range of one cyclotron energy $\omega_{c}=\frac{e B}{m}$ (where $m$ is the effective mass of the electron) in the two-dimensional density of states, that is $\frac{e B}{h}$ per LL. The number of the LLs filled with electrons is described by the filling factor $v=n \frac{h}{e B}$ (with $v=0$ corresponding to the neutral system). As $\nu$ is varied, for example by changing the applied magnetic field, the Landau level crossing the Fermi energy is filled or emptied of electrons. Repetitive crossing of the Fermi energy by Landau levels leads, for example, to oscillations in the conductivity measured as a function of the magnetic field (Shubnikov-de Haas effect) [129]. Similar in origin is the appearance of discrete steps in the Hall conductivity $\sigma_{x y}$ in the integer quantum Hall effect (QHE) [130, 131]. In fact, it is the observation of the unusual sequencing of these steps for monolayer and bilayer 
graphene $[10,11,54,132]$ that fuelled most of the initial interest in the Landau level structure of graphene systems. For monolayer, the sequence is shifted with respect to the QHE sequence of a 2DEG, so that $\sigma_{x y}= \pm g \frac{e^{2}}{h}\left(N+\frac{1}{2}\right)$, where $N$ is the Landau level index and $g$ is the level degeneracy (in graphene materials it is 4 due to double valley and double spin degeneracy). For bilayer, the steps appear at $\sigma_{x y}= \pm g \frac{e^{2}}{h} N$. However, the plateau at $\sigma_{x y}=0$ is missing. Both situations can be easily understood with the help of the Landau level structure. In particular, as the electronic structure of both systems is gapless (disregarding for a moment any on-site asymmetries), an addiditional, unusual Landau level is present at the energy $\epsilon=0$, sharing states between electrons and holes. Hence, both for monolayer and bilayer, no $v=0$ plateau exists in symmetric structures. For monolayer, integer filling factors follow then the sequence $v= \pm 2, \pm 6, \pm 10, \ldots$, leading to $\sigma_{x y}= \pm \frac{4 e^{2}}{h}\left(N+\frac{1}{2}\right)$. For bilayer, as we will show, the $\epsilon=0$ level contains twice as much electron states as other LLs. Thus, at least in strong magnetic fields, the plateau sequence $v= \pm 4, \pm 8, \pm 12, \ldots$, results in $\sigma_{x y}= \pm \frac{4 e^{2}}{h} N$.

One of the ways to study the Landau level spectrum of a (quasi-)twodimensional semiconductor heterostructure is to examine its optical absorption spectrum in an external magnetic field, usually perpendicular to the plane of the sample. This method, called simply magneto-optical (absorption) spectroscopy [133], has been extensively applied to graphene systems [59-68], mainly in relation to the unusual $\sqrt{B}$ dependence of the Landau level energy on the magnetic field and the physics of the zero-energy Landau level at very high fields. Review of the magneto-optical absorption spectroscopy of graphene systems is given in the broader context of optical properties of graphene multilayers in [19]. In monolayer graphene specifically, these studies confirmed the unequally spaced Landau level spectrum, arising from the linear electronic dispersion in the absence of the magnetic field, and the scaling of the Landau level energies as $\sqrt{B}$. Some deviations from the predictions of the tight-binding model 
with regards to the transition energies suggested contribution of many-particle interactions to the picture [60]. This is different from the two-dimensional systems with parabolic dispersion, where electron-electron interactions have no impact on the Landau level transition energies in magneto-optical experiments ('Kohn's theorem') [134]. For bilayer graphene, with the electronic dispersion quadratic at energies $\epsilon \ll \gamma_{1}$, the non-interacting theory predicts at low energies a linear scaling of the Landau level energy with the strength of the magnetic field [69]. At higher energies, $\epsilon \approx \gamma_{1}$, the Landau level energy should follow a $\sqrt{B}$-dependence. Thus, the Landau level spectrum of bilayer graphene should change from that characteristic of a parabolic dispersion to that of a linear dispersion. This has been observed experimentally by Henriksen et al. [63]. However, the changeover to a $\sqrt{B}$ behaviour occured at lower energies, and more suddenly, than expected. In fact, for some filling factors, a better fit was achieved when fitted to monolayer dispersion rather than bilayer one. Again, the many-body interactions were suggested as responsible for this departure from the predictions of the single particle theory.

In this chapter, we discuss the magneto-optical absorption spectroscopy of bilayer graphene and test the limits of the tight-binding approach as applied to the experimental situation of Henriksen and co-workers. In particular, we investigate the importance of the interlayer asymmetry in that experiment. This chapter is divided into three parts. In the first one, Sec. 4.1, we describe the Landau level structure of bilayer graphene using both the two-band and the four-band models. In this, we follow an approach applied to graphite [135137] and routinely used in the case of graphene systems [16, 51, 69]. We then derive in Sec. 4.2 selection rules for the optical absorption in magnetic field. We describe the optical strengths of transitions between any of the $\pi$ bands and include into the model presence of the physically most relevant asymmetries. We also show the resulting magneto-optical spectra. In the last part, Sec. 4.3, 
we concentrate on the role of interlayer asymmetry in the abovementioned experiment of Henriksen et al., results of which have been initially fitted to the predictions of the 'neutral bilayer' model (tight-binding model with the interlayer asymmetry equal to zero). We show that the experimental setup may have caused significant charge asymmetries between the layers and thus rendered the 'neutral bilayer' model unapplicable. We demonstrate that selfconsistently obtained values of the interlayer asymmetry in the presence of the magnetic field help to explain some of the discrepancies between experimental results and the theory used to interpret them. Some of the results contained in this chapter have been published in [71] and [73].

\subsection{Bilayer graphene in an external magnetic field}

\subsubsection{Landau levels in the two-band model}

In the following, we will work in the Landau gauge, $\boldsymbol{A}=(0, B x)$, and the resulting magnetic field

$$
B=\nabla \times A=(0,0, B)
$$

perpendicular to the sample. Let us consider a Landau function $\psi_{n, q}=e^{-i q y} \phi_{n}(x+$ $\left.q \lambda_{B}^{2}\right)$, where $\phi_{n}(x)$ is the eigenfunction of quantum harmonic oscillator. We then observe, that operators $\hat{\pi}=p_{x}+i p_{y}$ and $\hat{\pi}^{+}=p_{x}-i p_{y}$, with the the electronic momentum now containing the electromagnetic vector potential, $\boldsymbol{p}=-i \hbar \nabla-e \boldsymbol{A}$, coincide with the raising and lowering operators in the space of functions $\psi_{n, q}$. That is ${ }^{1}$,

$$
\begin{aligned}
& \hat{\pi}^{\dagger} \psi_{n}=i \frac{\hbar}{\lambda_{B}} \sqrt{2(n+1)} \psi_{n+1}, \\
& \hat{\pi} \psi_{n}=-i \frac{\hbar}{\lambda_{B}} \sqrt{2 n} \psi_{n-1},
\end{aligned}
$$

\footnotetext{
${ }^{1}$ From now on, we supress the index $q$ as irrelevant to our work.
} 
where the magnetic length $\lambda_{B}=\sqrt{\frac{\hbar}{e B}}$.

Looking now at the leading term in the two-band approximation,

$$
\hat{\boldsymbol{H}}_{\mathrm{eff}}=-\frac{v^{2}}{\gamma_{1}}\left(\begin{array}{cc}
0 & \left(\hat{\boldsymbol{\pi}}^{+}\right)^{2} \\
\hat{\boldsymbol{\pi}}^{2} & 0
\end{array}\right),
$$

we realise that the eigenstates for the above matrix can be written in a general form of $\Psi_{n}=\left(\psi_{n}, a \psi_{n-2}\right)$, with some complex coefficient $a$. In fact, after calculation we obtain a set of eigenvalues and eigenfunctions

$$
\begin{aligned}
& \epsilon_{n^{\alpha}}=\alpha \frac{2 \hbar^{2} v^{2}}{\gamma_{1} \lambda_{B}^{2}} \sqrt{n(n-1)} ; \\
& \Psi_{n^{\alpha}}=\left(\begin{array}{c}
\psi_{n} \\
0
\end{array}\right), n=0,1 ; \Psi_{n^{\alpha}}=\frac{1}{\sqrt{2}}\left(\begin{array}{c}
\psi_{n} \\
\alpha \psi_{n-2}
\end{array}\right), n \geq 2 ;
\end{aligned}
$$

where we use the index $n$ to number the Landau levels and $\alpha$ to distinguish between the valence $(\alpha=-1)$ and conduction $(\alpha=1)$ band. We see that at low energies, the energy of the Landau level is proportional to the strength of the magnetic field $B$. Also, for large $n$ the LLs are almost equidistant as $\sqrt{n(n-1)} \approx n$. Two levels, $n=0$ and $n=1$, have the same energy, $\epsilon_{0}=\epsilon_{1}=0$, giving rise to an unusual, 8-fold degenerate Landau level, which is shared between electrons and holes and thus does not require any index $\alpha$.

\subsubsection{Landau levels in the four-band model}

The derivation of the Landau level spectra within the four-band model follows very much the same approach as in the case of the two-band approximation. Again, we neglect $\gamma_{3}$ and other less important couplings. However, we add to our Hamiltonian the symmetry breaking on-site energies. In particular, we are interested in (i) the interlayer asymmetry $u$ and (ii) the substrate-induced intralayer asymmetry in the bottom layer only, $\delta$. The former is taken exactly as 
defined in Sec. 2.2 .3 while the latter can be obtained by taking $\Delta_{A B}=\Delta$, and reflects in the simplest way interaction of bilayer with an underlying substrate. In the case of bilayer graphene grown epitaxially on diatomic substrate, such as $\mathrm{SiC}$, this interaction distingiushes between sublattices in the bottom layer. However, due to a much greater distance, the interaction between the substrate and the top layer is neglected. The Hamiltonian in the linear approximation is then,

$$
\hat{\boldsymbol{H}}=\xi\left(\begin{array}{cccc}
\frac{u}{2}+\frac{\delta}{4}(1+\xi) & 0 & 0 & v \hat{\pi}^{+} \\
0 & -\frac{u}{2}-\frac{\delta}{4}(1-\xi) & v \hat{\pi} & 0 \\
0 & v \hat{\pi}^{\dagger} & -\frac{u}{2}+\frac{\delta}{4}(1-\xi) & \xi \gamma_{1} \\
v \hat{\pi} & 0 & \xi \gamma_{1} & \frac{u}{2}-\frac{\delta}{4}(1+\xi)
\end{array}\right)
$$

In this case, we construct the eigenstates using functions $\psi_{n}$ and $\psi_{n-2}$ (for the first two components of the eigenstate), as well as $\psi_{n-1}$ (for the last two components). In the following, we use superscript $c(s)$ to denote Landau levels originating from the low-energy (high-energy) bands and $\alpha$ equal to 1 or -1 to indicate the sign of the energy. Then, in the case of symmetric bilayers $(u, \delta=0)$, the energy $\epsilon_{n \alpha}^{c}$ of the $n$-th low-energy Landau level is given by:

$$
\begin{aligned}
& \epsilon_{0}^{c}=\epsilon_{1}^{c}=0 \\
& \epsilon_{n \alpha}^{c}=\frac{\alpha}{\sqrt{2}}\left(\gamma_{1}^{2}+2 \frac{\hbar^{2} v^{2}}{\lambda_{B}^{2}}(2 n-1)-\sqrt{\gamma_{1}^{4}+\frac{4 \hbar^{2} v^{2} \gamma_{1}^{2}}{\lambda_{B}^{2}}(2 n-1)+\frac{4 \hbar^{4} v^{4}}{\lambda_{B}^{4}}}\right)^{\frac{1}{2}} \text { for } n \geq 2 .
\end{aligned}
$$

In turn, the energy $\epsilon_{n \alpha}^{s}$ in the high-energy bands is

$$
\epsilon_{n \alpha}^{s}=\frac{\alpha}{\sqrt{2}}\left(\gamma_{1}^{2}+2 \frac{\hbar^{2} v^{2}}{\lambda_{B}^{2}}(2 n-1)+\sqrt{\gamma_{1}^{4}+\frac{4 \hbar^{2} v^{2} \gamma_{1}^{2}}{\lambda_{B}^{2}}(2 n-1)+\frac{4 \hbar^{4} v^{4}}{\lambda_{B}^{4}}}\right)^{\frac{1}{2}} \text { for } n \geq 1
$$

In this formulation, for high-energy LLs indexing starts with $n=1$, not $n=0$, emphasizing the distinctiveness of the $\epsilon=0 \mathrm{LL}$. Each level has additional 4 -fold 
degeneracy due to valleys and spins.

For magnetic fields $B<20 \mathrm{~T}$, expressions for the LL energy in a symmetric bilayer, Eqs. (4.5) and (4.6), can be simplified using a small parameter $x=$ $\frac{\sqrt{2} \hbar v}{\lambda_{B} \gamma_{1}} ;|x| \ll 1$. Additionally, assuming that $u$ and $\delta$ are small, we obtain LL energies for asymmetric (charged) bilayer:

$$
\begin{aligned}
& \epsilon_{0}^{c}=\xi\left[\frac{u}{2}+\frac{\delta}{4}(\xi+1)\right] ; \epsilon_{1}^{c} \approx \epsilon_{0}^{c}-\xi x^{2}\left(u+\xi \frac{\delta}{2}\right) ; \\
& \epsilon_{n \alpha}^{c} \approx \Sigma+\alpha \sqrt{\epsilon_{n}^{2}+\Omega^{2}}, n \geq 2 \\
& \epsilon_{n \alpha}^{s} \approx \alpha\left|\gamma_{1}\right|\left(1+x^{2}\left(n-\frac{1}{2}\right)\right)+\frac{1}{2}\left[\xi u x^{2}-\frac{\delta}{2}\left(1-x^{2}(2 n-1)\right)\right], n \geq 1
\end{aligned}
$$

where

$$
\begin{aligned}
& \Sigma=\frac{\delta}{4}-\frac{\xi x^{2}}{2}\left[u(2 n+1)+\frac{\delta}{2}(2 n-1)(2 \xi+1)\right] ; \\
& \Omega=\frac{\xi}{2}\left(u+\frac{\delta}{2}\right)-\frac{x^{2}}{2}\left[\xi u(2 n-1)+\frac{\delta}{4}(2 n+1)\right] ; \epsilon_{n}=\left|\gamma_{1}\right| x^{2} \sqrt{n(n-1)} .
\end{aligned}
$$

The numerically calculated Landau level spectra both in the absence and presence of the asymmetries $u$ and $\delta / 2$ are shown in Fig. 4.1(a)-(d). The lowenergy Landau level spectrum for neutral bilayer, equivalent with Eq. (4.5), is shown as grey solid lines in Fig. 4.1(a)-(d). Those levels create a fan-plot originating at zero energy. As shown earlier within the two-band approximation, levels $n=0$ and $n=1$ have the same energy, leading to an unusual 8 -fold degenerate level at $\epsilon=0$. The high-energy LLs, Eq. (4.6), create two additional fan-plots originating at $\epsilon= \pm \gamma_{1}$, which are shown together with low-energy LLs with grey solid lines in Fig. 4.1(a)-(b). The Landau level spectra for $u=50 \mathrm{meV}$ $\left(\frac{\delta}{2}=50 \mathrm{meV}\right)$ is shown with black lines in Fig. 4.1(b) and (d) [(a) and (c)]. Both of the asymmetries lift the valley degeneracy. Also, in both cases the additional degeneracy of $n=0$ and $n=1 \mathrm{LLs}$ is removed, $\epsilon_{1} \neq \epsilon_{0}$ [note that even the $n=0$ level is shifted with respect to the middle of the gap at the $K$ point from $\epsilon=0$ in opposite directions in the valley $K_{+}(\xi=+)$ and $\left.K_{-}(\xi=-)\right]$. As can be seen in 

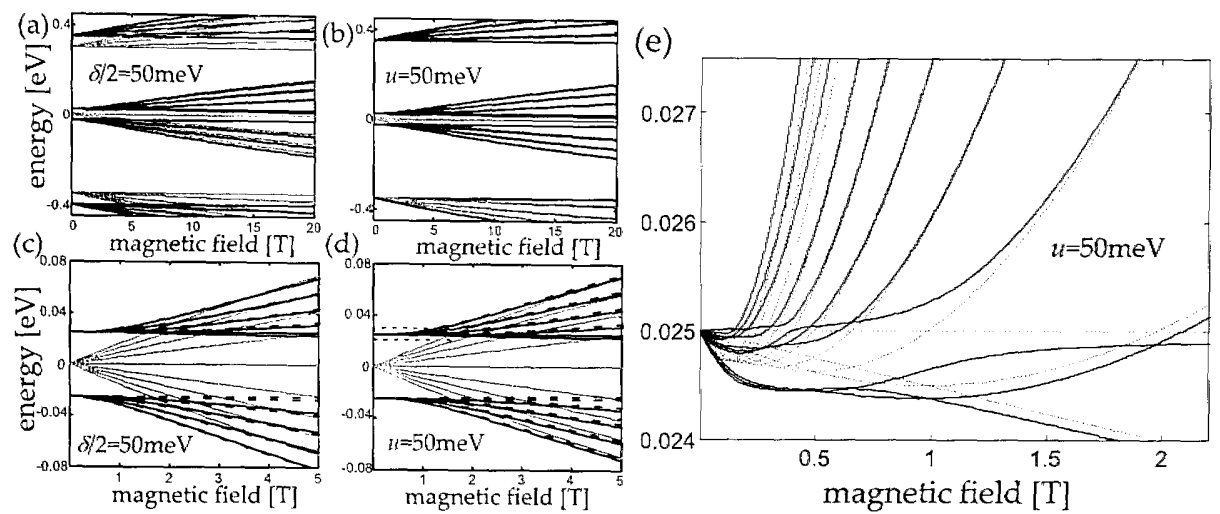

Figure 4.1: Numerically calculated Landau levels of bilayer graphene for range of high [(a) and (b)] and low [(c) and (d)] energies; black solid and dashed lines in (c) and (d) represent levels at valley $K_{+}$and $K_{-}$respectively, grey solid lines in the background show the Landau level spectrum for no asymmetries $(u, \delta=0)$; in (a) and (b) only the spectrum at $K_{+}$is shown for clarity. Note that nonzero $u$ in (b) affects the high-energy LLs very weakly and so the corresponding grey lines are underneath the black ones. Zero of the energy scale is shifted to the middle of the gap opened in each case at the K point. (e) Area bounded by dashed red rectangle in (d) shown again with the 11 lowest LLs at $\mathrm{K}+$ : black solid lines - taking into account $v_{3}=0.15 \mathrm{eV}$, grey dashed lines $-v_{3}$ neglected. Values of parameters used: $v=1 \times 10^{6} \mathrm{~m} / \mathrm{s}, \gamma_{1}=0.35 \mathrm{eV}$, resulting in low-energy effective mass $m_{\mathrm{eff}}=\gamma_{1} / 2 v^{2} \approx 0.03$ of the electron mass.

Fig. 4.1(a), interlayer asymmetry $u$ affects the high-energy LLs very weakly and so, the corresponding black lines cover grey lines showing high-energy LLs in the symmetric bilayer. At low energies and low fields [Fig. 4.1(e)], signatures of a Mexican hat developing in the electronic spectrum of an asymmetric bilayer can be noticed in the fan-plots of the LL spectrum. Inverted curvature in the central part of such a structure (hole-like in conduction and electron-like in valence band) results in inverted behaviour of Landau levels at very low $B$ [the energy of electron (hole) levels decrease (increase) with increasing B] which then returns to typical behaviour at higher B [the energy of electron (hole) levels increase (decrease) with increasing B]. This results in interlevel crossings. Also, this is a regime where the influence of the parameter v3, neglected so far, is important, because it mixes LLs $n$ and $n-3$, thus changing some of the interlevel crossings into anticrossings. An example of the numerically calculated spectrum taking into account $v_{3}$ using a procedure explained in the next section is shown in Fig. 
2(e).

The wave functions corresponding to LLs described in Eqs. (4.7) are as follows:

$$
\begin{aligned}
& \psi_{0}^{c}=\left(\begin{array}{c}
-i \xi \psi_{0} \\
0 \\
0 \\
0
\end{array}\right) ; \psi_{1}^{c} \approx\left(\begin{array}{c}
-i \xi\left(1-\frac{x^{2}}{2}\right) \psi_{1} \\
0 \\
x \psi_{0} \\
0
\end{array}\right) ; \\
& \psi_{n \beta}^{c} \approx \frac{1}{2 \sqrt{\Gamma_{n}\left(\Gamma_{n}+\epsilon_{n}\right)}}\left(\begin{array}{c}
-i \xi\left[1-x^{2}\left(n-\frac{1}{4}\right)\right]\left(\Gamma_{n}+\epsilon_{n}+\beta \Omega\right) \psi_{n} \\
-i \xi \beta\left(1+\frac{x^{2}}{4}\right)\left(\Gamma_{n}+\epsilon_{n}-\beta \Omega\right) \psi_{n-2} \\
x \sqrt{n}\left(\Gamma_{n}+\epsilon_{n}+\beta \Omega\right) \psi_{n-1} \\
-\beta x \sqrt{n-1}\left(\Gamma_{n}+\epsilon_{n}-\beta \Omega\right) \psi_{n-1}
\end{array}\right), n \geq 2 ; \\
& \psi_{n \beta}^{s} \approx \frac{1}{\sqrt{2}\left(\begin{array}{c}
i \xi x \sqrt{n} \psi_{n} \\
-i \xi \beta x \sqrt{n-1} \psi_{n-2} \\
\left(1-\frac{n x^{2}}{2}\right) \psi_{n-1} \\
\beta\left[1-\frac{x^{2}}{2}(n-1)\right] \psi_{n-1}
\end{array}\right), n \geq 1 ;}
\end{aligned}
$$

where:

$$
\beta= \pm 1 ; \Gamma_{n}=\sqrt{\epsilon_{n}^{2}+\Omega_{n}^{2}}
$$

\subsubsection{Numerical treatment of the $\gamma_{3}$ coupling}

The derivation of the Landau level structure of bilayer graphene around a single valley, as shown in previous sections, is possible because each of the components of the wave function can be written in terms of only one Landau function $\psi_{n}$. Any of the on-site asymmetries, as well as the couplings $\gamma_{0}, \gamma_{1}$ or $\gamma_{4}$ can be incorporated into this scheme. However, the $\gamma_{3}$ coupling leads to mixing of the LLs with each other and the problem can no longer be solved in this way. This perturbation becomes important only at weak magnetic fields and in most situations, it can be neglected for fields $B>5$ T. Nevertheless, we investigate here 
how the $\gamma_{3}$ terms affect the Landau level structure of bilayer graphene at weak magnetic fields. This is because with improving sample fabrication processes, it is now possible to probe the physics at energies where $\gamma_{3}$ is important [138]. We use an approach similar to that developed to treat $\gamma_{3}$ in the calculation of the LL spectrum of graphite [137] and for simplicity, discuss the case of the two-band model, Eq. (2.12).

In an external magnetic field, the $\gamma_{3}$ perturbation mixes LLs with each other so that the electron amplitude on each sublattices is now a linear combination of infinitely many functions $\psi_{n}$. Using the knowledge from the previous sections, we again want to describe the eigenstates with vectors whose odd and even components correspond to electron wave amplitudes on one of the sublattices, $B 2(A 1)$ and $A 1(B 2)$ in the $K_{+}\left(K_{-}\right)$valley, respectively. Also, we want each component to be expressed using a single function $\psi_{n}$. We choose the ordering of the entries corresponding to specific $\psi_{n}$ in such a way, so that pair of vectors $(2 n-1)$-th and $(2 n)$-th create a minimal subspace required to describe the $n$-th LL in the absence of $\gamma_{3}$. In the absence of $\gamma_{3}$, this tallies to repeating Hamiltonian (4.2) as the diagonal block of an infinite matrix, with all other elements equal to zero. The presence of $\gamma_{3}$ leads to some off-diagonal perturbations in this matrix, which can be written in the form ${ }^{2}$ :

$$
\hat{\boldsymbol{H}}=\left(\begin{array}{cccccc}
0 & 0 & 0 & \hat{\boldsymbol{D}}(1) & 0 & \cdots \\
0 & 0 & 0 & 0 & \hat{D}(2) & \cdots \\
0 & 0 & \hat{\boldsymbol{H}}(1) & 0 & 0 & \cdots \\
\hat{D}^{\dagger}(1) & 0 & 0 & \hat{\boldsymbol{H}}(2) & 0 & \cdots \\
0 & \hat{\boldsymbol{D}}^{\dagger}(2) & 0 & 0 & \hat{\boldsymbol{H}}(3) & \cdots \\
\vdots & \vdots & \vdots & \vdots & \vdots & \ddots
\end{array}\right),
$$

\footnotetext{
${ }^{2}$ Note, that two rows and columns in the following matrix, identically equal to zero, give rise to two solutions at zero energy, which correspond to unphysical eigenstates (zero vectors) and should not be confused with the zero energy Landau levels described earlier in the text.
} 




Figure 4.2: Comparison of the low-energy and weak-field Landau level structures obtained neglecting the $\gamma_{3}$ coupling (black lines) and taking it into account (red lines). Values of parameters used: $v=1 \times 10^{6} \mathrm{~m} / \mathrm{s}, \gamma_{1}=0.4 \mathrm{eV}$, resulting in low-energy effective mass $m_{\mathrm{eff}}=\gamma_{1} / 2 v^{2} \approx 0.03$ of the electron mass, $v_{3}=0.1 v$ (if applicable).

where

$$
\hat{\boldsymbol{H}}(n)=\left(\begin{array}{cc}
0 & \frac{v^{2}}{\gamma_{1}} x^{2} \sqrt{n(n+1)} \\
\frac{v^{2}}{\gamma_{1}} x^{2} \sqrt{n(n+1)} & 0
\end{array}\right), \hat{\boldsymbol{D}}(n)=\left(\begin{array}{cc}
0 & -i \xi x v_{3} \sqrt{n} \\
0 & 0
\end{array}\right) .
$$

We then truncate the infinite basis, restricting the calculation to a given $n$ LLs and diagonalise the resulting Hamiltonian numerically. The number of the basis vectors required in the calculation in order to describe properly the lowenergy LL structure increases with decreasing magnetic field (reflecting growing importance of the $\gamma_{3}$ terms at weaker fields). Basis of of the dimension 800 is enough to describe LL spectra for magnetic fields $B>0.01 \mathrm{~T}$. Similar analysis can be performed within the four-band model. However, the matrix dimension has to be then doubled for the same accuracy at low energies, whereas the correction to the high energy LLs is negligible.

The low-energy Landau level spectra at weak fields has been presented in Fig. 4.2. The black lines show the LL spectrum equivalent to that described in Eq. (4.3), whereas the red lines demonstrate the low-energy LL spectrum with 
the $\gamma_{3}$ taken into account (via a procedure explained above). Only the energies of few lowest Landau levels are affected. The unusual degeneracy of the $\epsilon=0$ Landau level is preserved.

\subsection{Magneto-optical selection rules and the absorp- tion spectra}

We describe the electron-photon interaction within the four-band linear approximation, by expanding the Hamiltonian $\hat{H}_{\xi}(p-e A)$, where $[p-e A]$ is the canonical momentum including the electromagnetic vector potential $\boldsymbol{A}$, up to the first power in $A$, and write the interaction Hamiltonian

$$
\hat{H}_{\text {int }}=\hat{j} \cdot A,
$$

where $\hat{j}=-e\left(\frac{\partial \hat{H}_{\xi}}{\partial q_{x}}, \frac{\partial \hat{H}_{\xi}}{\partial q_{y}}\right)$ is the current operator. The incoming beam is characterised by a time-dependent electric field, $\boldsymbol{E}_{\omega}(t)=\boldsymbol{E}_{\omega} e^{-i \omega t}$. Using Maxwell's equations, we arrive at

$$
A=\frac{1}{-i \omega} E_{\omega} e^{-i \omega t}
$$

Wave functions given in Eqs. (4.9) can now be used to determine transition rules for the absorption of right $(\oplus)$ and left-handed $(\ominus)$ circularly polarized light $\boldsymbol{E}_{\omega}=E_{\omega} l_{\oplus / \ominus}$, with $l_{\oplus}=\frac{1}{\sqrt{2}}\left(l_{x}-i l_{y}\right)$ and $l_{\ominus}=\frac{1}{\sqrt{2}}\left(l_{x}+i l_{y}\right)$. Neglecting for now the prefactor $\frac{E_{\omega}}{-i \omega}$ in the interaction above, we end with perturbation $\left(\hat{j} \cdot l_{\oplus / \theta}\right)$ for circularly polarised light interacting with electrons in the material. We can now find optical strengths of inter-LL transitions. We give here all of them with the accuracy up to the first meaningful term in $\frac{1}{\gamma_{1}}$. For the transitions between the 
low-energy bands $(c \rightarrow c)$, we obtain:

$$
\begin{aligned}
& \left|\left\langle\psi_{1}^{c}\left|\hat{j} \cdot l_{\oplus}\right| \psi_{0}^{c}\right\rangle\right|^{2}=\left|\left\langle\psi_{0}^{c}\left|\hat{j} \cdot l_{\ominus}\right| \psi_{1}^{c}\right\rangle\right|^{2} \approx \frac{e^{2} v^{2} x^{2}(2 \xi u+\delta)^{2}}{2 \gamma_{1}^{2}}, \\
& \left|\left\langle\psi_{2 \beta}^{c}\left|\hat{j} \cdot l_{\oplus}\right| \psi_{1}^{c}\right\rangle\right|^{2} \approx 2 e^{2} v^{2} x^{2} \Lambda_{2 \beta}^{(-)}, \quad\left|\left\langle\psi_{1}^{c}\left|\hat{j} \cdot l_{\ominus}\right| \psi_{2 \alpha}^{c}\right\rangle\right|^{2} \approx 2 e^{2} v^{2} x^{2} \Lambda_{2 \alpha}^{(-)}, \\
& \left|\left\langle\psi_{n \beta}^{c}\left|\hat{j} \cdot l_{\oplus}\right| \psi_{m \alpha}^{c}\right\rangle\right|^{2} \approx e^{2} v^{2} x^{2} m \delta_{n-1, m} \frac{\Lambda_{m \alpha}^{(+)} \Lambda_{n \beta}^{(-)}}{2}, m \geq 2, \\
& \left|\left\langle\psi_{n \beta}^{c}\left|\hat{j} \cdot l_{\ominus}\right| \psi_{m \alpha}^{c}\right\rangle\right|^{2} \approx e^{2} v^{2} x^{2}(m-1) \delta_{n, m-1} \frac{\Lambda_{m \alpha}^{(-)} \Lambda_{n \beta}^{(+)}}{2}, m \geq 3 .
\end{aligned}
$$

For the transitions between the low-energy and the high-energy bands $(s \leftrightarrow c)$, we have:

$$
\begin{aligned}
& \left|\left\langle\psi_{n \beta}^{s}\left|\hat{j} \cdot l_{\oplus}\right| \psi_{m}^{c}\right\rangle\right|^{2} \approx e^{2} v^{2} \delta_{n-1, m}, m=0,1, \\
& \left|\left\langle\psi_{n \beta}^{s}\left|\hat{j} \cdot l_{\oplus}\right| \psi_{m \alpha}^{c}\right\rangle\right|^{2} \approx \frac{e^{2} v^{2} \Lambda_{m \alpha}^{(+)}}{4} \delta_{n-1, m}, m \geq 2, \\
& \left|\left\langle\psi_{n \beta}^{s}\left|\hat{j} \cdot l_{\ominus}\right| \psi_{m \alpha}^{c}\right\rangle\right|^{2} \approx \frac{e^{2} v^{2} \Lambda_{m u \psi}^{(-)}}{4} \delta_{n, m-1}, \quad m \geq 2, \\
& \left|\left\langle\psi_{n \beta}^{c}\left|\hat{j} \cdot l_{\oplus}\right| \psi_{m \alpha}^{s}\right\rangle\right|^{2} \approx \frac{e^{2} v^{2} \Lambda_{n \beta}^{(-)}}{4} \delta_{n-1, m}, m \geq 1, \\
& \left|\left\langle\psi_{n}^{c}\left|\hat{j} \cdot l_{\ominus}\right| \psi_{m \alpha}^{s}\right\rangle\right|^{2} \approx e^{2} v^{2} \delta_{n, m+1}, m=1,2, \\
& \left|\left\langle\psi_{n \beta}^{c}\left|\hat{j} \cdot l_{\ominus}\right| \psi_{m \alpha}^{s}\right\rangle\right|^{2} \approx \frac{e^{2} v^{2} \Lambda_{n \beta}^{(+)}}{4} \delta_{n, m-1}, m \geq 3 .
\end{aligned}
$$

Finally, for the transitions among the high-energy bands alone $(s \rightarrow s)$, we have:

$$
\begin{aligned}
& \left|\left\langle\psi_{n \beta}^{s}\left|\hat{j} \cdot l_{\oplus}\right| \psi_{m \alpha}^{s}\right\rangle\right|^{2} \approx 2 e^{2} v^{2} x^{2} m \delta_{n-1, m}, \quad m \geq 1, \\
& \left|\left\langle\psi_{n \beta}^{s}\left|\hat{j} \cdot l_{\ominus}\right| \psi_{m \alpha}^{s}\right\rangle\right|^{2} \approx 2 e^{2} v^{2} x^{2}(m-1) \delta_{n, m-1}, \quad m \geq 2 .
\end{aligned}
$$

In the above equations, we introduced:

$$
\Lambda_{m \alpha}^{(\mu)}=\frac{\left(\Gamma_{m}+\epsilon_{m}+\mu \alpha \Omega\right)^{2}}{\Gamma_{m}\left(\Gamma_{m}+\epsilon_{m}\right)}, \quad \Lambda_{m \alpha}^{(\mu)}=2 \text { for } u, \delta=0 .
$$

Equations in (4.17) generalise the earlier study of optical and magneto-optical absorption in bilayers [69]. Examples of allowed transitions are illustrated in 
Fig. 4.3(a)-(b). Independently of the presence/absence of asymmetries $\delta$ or $u$, selection rules for absorption of right-handed polarized light are such that the Landau level index has to be decreased by one, whereas absorption of lefthanded photons requires an increase of the Landau level index by one. Also, optical strengths of $c \rightarrow c$ and $s \rightarrow s$ transitions are proportional to the magnetic field $B$ and LL index, whereas optical strengths of $s \leftrightarrow c$ transitions are almost independent of $B$. As $x$ is a small parameter, the intensity of the first $s \leftrightarrow c$ transitions should be higher than for $c \rightarrow c$ transitions corresponding to the same energy of incident radiation $\hbar \omega$.

We describe the optical absorption of the incoming photons by the sample in the presence of an external magnetic field, $I_{\mathrm{abs}}$, by the ratio of the energy absorbed by the material to the energy carried by the electromagnetic field, $\langle S\rangle \propto E_{\omega}^{2}$. The energy absorbed is proportional to the energy of the incoming photons, density of electrons in the Landau level (and thus to the strength of the magnetic field $B)$ and the transition probability between the initial and final states, expressed by the optical strengths above. One also has to take into account electron level occupancy $f_{n}$ of the level $|n\rangle$ and conservation of energy in the process. Hence, the intensity of absorption, $I_{\mathrm{abs}}$, is proportional to

$$
I_{\mathrm{abs}}(\omega) \propto \sum_{m, n} \frac{\left(\epsilon_{n}-\epsilon_{m}\right)\left(f_{m}-f_{n}\right)\left|\left\langle n\left|\hat{j} \cdot l_{\oplus / \ominus}\right| m\right\rangle\right|^{2} B}{\omega^{2}} \delta\left(\epsilon_{n}-\epsilon_{m}-\hbar \omega\right)
$$

where $|m\rangle$ and $|n\rangle$ are the initial and final electron states, respectively. In Fig. $4.3(\mathrm{c})$, the numerically calculated magneto-optical spectrum of symmetric bilayer for $\hbar \omega \approx\left|\gamma_{1}\right|$ has been shown (the range of energies at which the $s \leftrightarrow c$ transitions become possible). For this purpose, we approximated the Dirac delta expressing the energy conservation with a Lorentzian, with the full width at half-maximum parameter $\Gamma$ associated with the broadening of the Landau levels (as the incoming photon beam is monochromatic). We assumed the same 

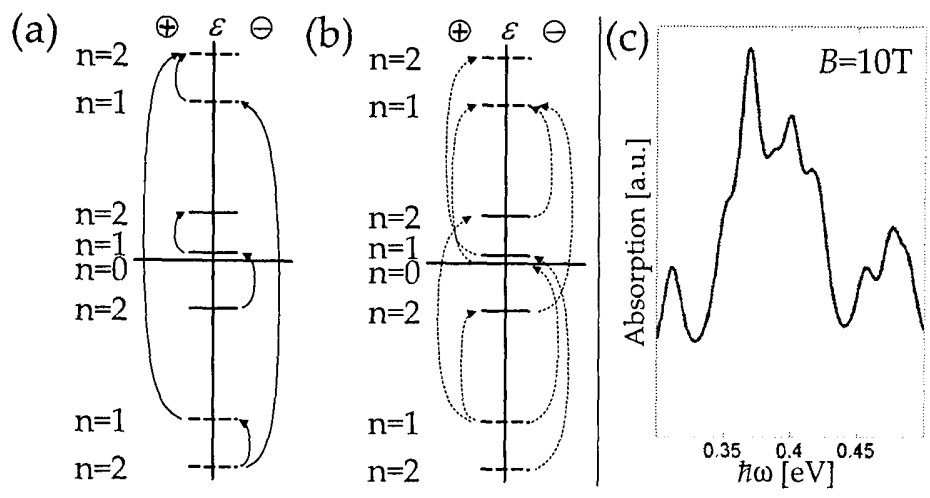

Figure 4.3: Allowed intraband (a) and interband (b) optical excitations. Lowenergy (high-energy) LLs are depicted with solid (dashed) lines. Transitions in $\mathbf{1}_{\oplus}\left(\mathbf{1}_{\ominus}\right)$ are shown on the left (right) of the energy axis, under $\oplus(\ominus)$ symbol. Energy axis not to scale; not shown is very weak $\left|0_{c}\right\rangle \rightarrow\left|1_{c}\right\rangle$ transition allowed only in the presence of asymmetry. (c) Magneto-optical absorption spectrum for the energy of incident light $\hbar \omega \approx\left|\gamma_{1}\right|$; magnetic field $B=10 \mathrm{~T}, \gamma_{1}=-0.35 \mathrm{eV}$, $v=10^{6} \mathrm{~m} / \mathrm{s}$; Landau level broadening was approximated with a Lorentzian with full width at half maximum $20 \mathrm{meV}$.

broadening of all Landau levels. The onset of $s \leftrightarrow c$ transitions (the two highest peaks around $\hbar \omega \approx 0.39 \mathrm{eV}$ ) can be observed against the background of $c \rightarrow c$ excitations.

\subsection{Magneto-Optical spectroscopy in charged bilayer graphene}

\subsubsection{Landau level spectrum in charged bilayer graphene: self- consistent analysis of the interlayer asymmetry gap}

In the infrared experiment realised by Henriksen and co-workers [63], interLL transitions for the filling factors $v= \pm 4, \pm 8, \pm 12, \pm 16$ have been separately traced for increasing strength of the applied external magnetic field. The observed behaviour of the transition energies followed the predictions of the noninteracting model, that is, showed a change from a linear dependence of the LL energy on $B$ to a $\sqrt{B}$ dependence. However, the changeover occured at lower 
energies, and more suddenly, than expected. For some filling factors, a better fit was achieved when fitted to monolayer dispersion rather than bilayer one. Many-body interactions were suggested as responsible for this departure from the predictions of the single particle theory. However, the experimental setup and the need to fix the filling factor while the magnetic field was changed to trace a particular transition, implies significant changes in the carrier density in bilayer graphene during the experiment by applying an external electric field perpendicular to the layers. Such external electric field is known to induce interlayer asymmetry in the system $[72,139,140]$, in our model described by the on-site energy $u$ (see Sec. 2.2.3). Non-zero asymmetry $u$, caused by a possible difference in electric potential energy between the layers, opens a gap in the electronic spectrum and, in the presence of an additional external magnetic field, modifies the LL spectrum [51, 71, 141-143]. To model this effect, we employ a self-consistent theory of the charging of bilayer graphene. In order to reproduce the experimental conditions, we extend the self-consistent analysis of Ref. [72] from the zero-magnetic field regime into the regime of quantizing magnetic fields, taking into account the possibility that there is a finite asymmetry in a neutral structure [see Eq. (4.20) below].

In particular, we consider a gated bilayer as shown in Fig. 4.4. The interlayer distance is $c_{0}$. In an external magnetic field $B$, the Landau levels are described by the four-band Hamiltonian, Eq. (4.4). We neglect from now on the interlayer assymmetry $\delta$. In order to keep the filling factor $v$ fixed while changing $B$, a total excess density, $n=v \frac{e B}{h}$, must be induced using the gate. The density $n$ is shared between the two layers: $n=n_{1}+n_{2}$, where, assuming a top gate, $n_{2}\left(n_{1}\right)$ is the excess density on the layer closest to (furthest from) the gate. The interlayer asymmetry $u$ is a result of different electric potentials $U_{1}^{\text {pot }}$ and $U_{2}^{\text {pot }}$ and the first and second layer and is therefore related to the electric field $E$ between the layers which arises due to the incomplete screening of the gate electric field by 




Figure 4.4: Schematic of a 'biased bilayer' showing all charge densities and electric fields induced by a single gate.

the charge $e n_{2}$ on the top layer alone. Hence, with the help of Gauss's law, the interlayer asymmetry $u$ can be related to the unscreened density $n_{1}$,

$$
u(v, B)=w+\frac{e^{2} c_{0} n_{1}(v, B)}{\epsilon_{0} \epsilon_{r}} .
$$

Here, $\epsilon_{r}$ is the effective dielectric constant determined by the $\mathrm{SiO}_{2}$ substrate, and $w$ takes into account finite asymmetry of a neutral structure (internal electric field due to, for example, initial non-intentional doping of the flake by deposits/adsorbates). In our numerical calculations we use $\epsilon_{r}=2$.

On the one hand, $u$ influences the LL spectrum via the Hamiltonian in Eq. (4.4). On the other hand, its value depends on the charge density $n_{1}$ which can only be obtained with a full knowledge of the LL spectrum and the wave functions corresponding to each LL. Therefore, a calculation of $u$ requires a selfconsistent numerical analysis. This calculation consists of the following steps: for each given $B, 5<B<20 \mathrm{~T}$, and $v$ we choose a starting $u$, and diagonalize the 
(a)
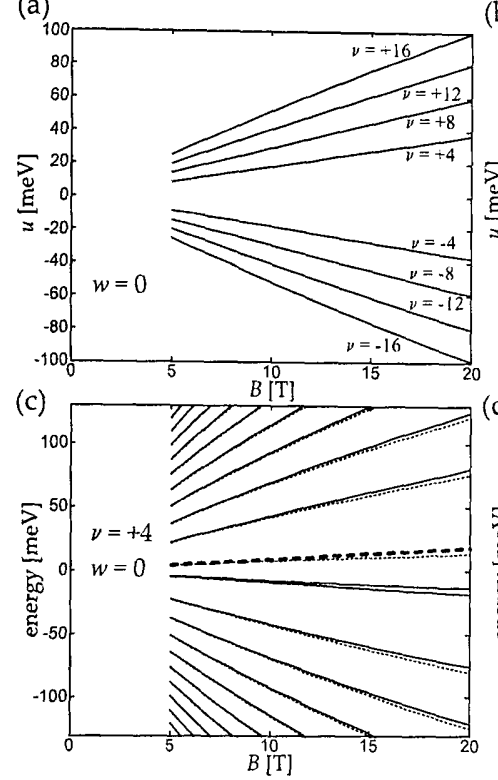

(b)
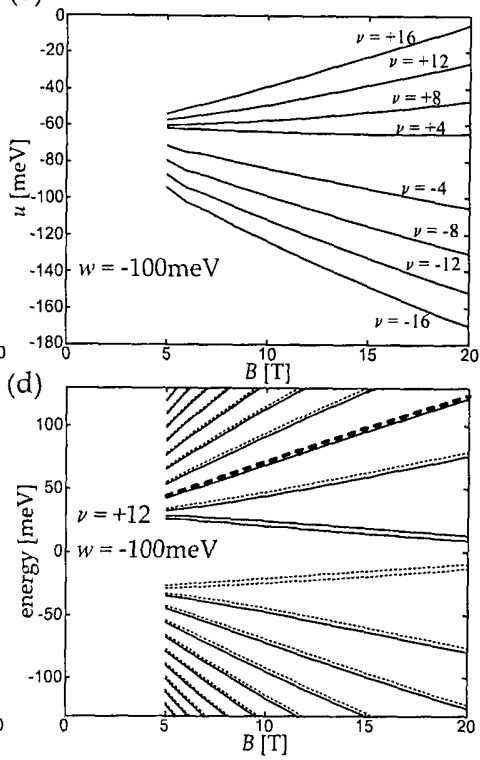

Figure 4.5: Top row: results of a self-consistent calculation of the interlayer asymmetry $u$ for (a) $w=0$ and (b) $w=-100 \mathrm{meV}$. Bottom row: the LL spectrum as a function of applied magnetic field $B$ for constant filling factor and excess density-induced interlayer asymmetry $u$ : (c) $v=+4 ; w=0$, and (d) $v=+12$; $w=-100 \mathrm{meV}$. Dashed and solid lines denote levels belonging to $K_{+}$and $K_{-}$, respectively. The line corresponding to the last filled Landau level is shown in bold. In these calculations we used $v=10^{6} \mathrm{~m} / \mathrm{s}$ and $\gamma_{1}=0.4 \mathrm{eV}$.

Hamiltonian to find the LL spectrum and the eigenstates with $m \lesssim M_{\max }$ where $M_{\max } \sim 300$. Then, we sum over all filled Landau levels and determine the excess electron densities on each layer. Note that, as a nonzero value of $u$ splits the valley degeneracy of the LLs [71], care has to be taken when comparing densities in specific LLs in biased and neutral structures, not to confuse levels in different valleys. Finally, using Eq. (4.20) we find the asymmetry parameter and, then, iterate the numerical procedure to obtain the self-consistent value of $u .^{3}$ Note that, for a sufficiently large cutoff $M_{\max } \sim 300$, the results were independent of $M_{\max }$.

The self-consistently calculated values of $u$ obtained for several values of the filling factor $v$ are shown in Fig. 4.5(a) and (b) for the case of $w=0$ and a nominal $w=-100 \mathrm{meV}$, respectively. In the case when $w=0$, the induced

\footnotetext{
${ }^{3}$ Neither the Landau level broadening nor partial filling factors were considered in this work.
} 
interlayer asymmetry is antisymmetric with respect to the change of the filling factor from positive to negative. This is because changing the filling factor from $+v$ to $-v$ corresponds to reversing the applied electric field and inducing excess densities $-n,-n_{1}$ and $-n_{2}$ and thus reversing the sign of $u$. Also, with decreasing $B$ all curves tend towards $u=0$ and $u \approx-60 \mathrm{meV}$ in Fig. 2(a) and 2(b), respectively. These values are the results of the self-consistent calculation with corresponding values of $w$ in the absence of a magnetic field [72]. Examples of the low-energy LL spectrum for $v=+4, w=0$ and for $v=+12, w=-100 \mathrm{meV}$ are shown in Fig. 4.5(c) and (d). To refer to LLs as shown in Fig. 4.5, we use in the further text three symbols: $\operatorname{si\xi } \xi$, where $s$ attributes the LL to the conduction $(+)$ or valence $(-)$ band, $m$ is the $L L$ index and $\xi \in(+,-)$ identifies the valley $\left(K_{+}\right.$or $K_{-}$) that the level belongs to, respectively. As mentioned in the previous sections, the Landau levels $m=0,1$ have no $s$ index, as those levels are degenerate when $u=0$. The sign of the valley splitting of the level sm depends on the sign of $u$ : for $u>0$, level $s m$ - has higher energy than level $s m+$ whereas the opposite is true for $u<0$. Levels $m=0,1$ behave differently - in this case

$$
\left.\left.\begin{array}{l}
\epsilon_{m+}>0 \\
\epsilon_{m-}<0
\end{array}\right\} \text { for } u>0, \quad \begin{array}{l}
\epsilon_{m+}<0 \\
\\
\epsilon_{m-}>0
\end{array}\right\} \text { for } u<0
$$

The size of the valley splitting of the low-energy LLs increases with $u$ and $B$ and for $|u| \approx 0.1 \mathrm{eV}, B \approx 20 \mathrm{~T}$ [filling factors $v=+12,+16$ in Fig. 4.5(a) and $v=-8,-12,-16$ in Fig. $4.5(\mathrm{~b})]$ is of the order of $10 \mathrm{meV}$.

\subsubsection{Tracking a single inter-LL transition: low-energy inter- Landau level transitions}

Using spectra similar to those shown in Fig. 4.5(c) and (d), we find the energy of the low-energy inter-Landau level transitions for several filling factors which mimics the experimental conditions of Ref. [63] (the tight-binding approach to 

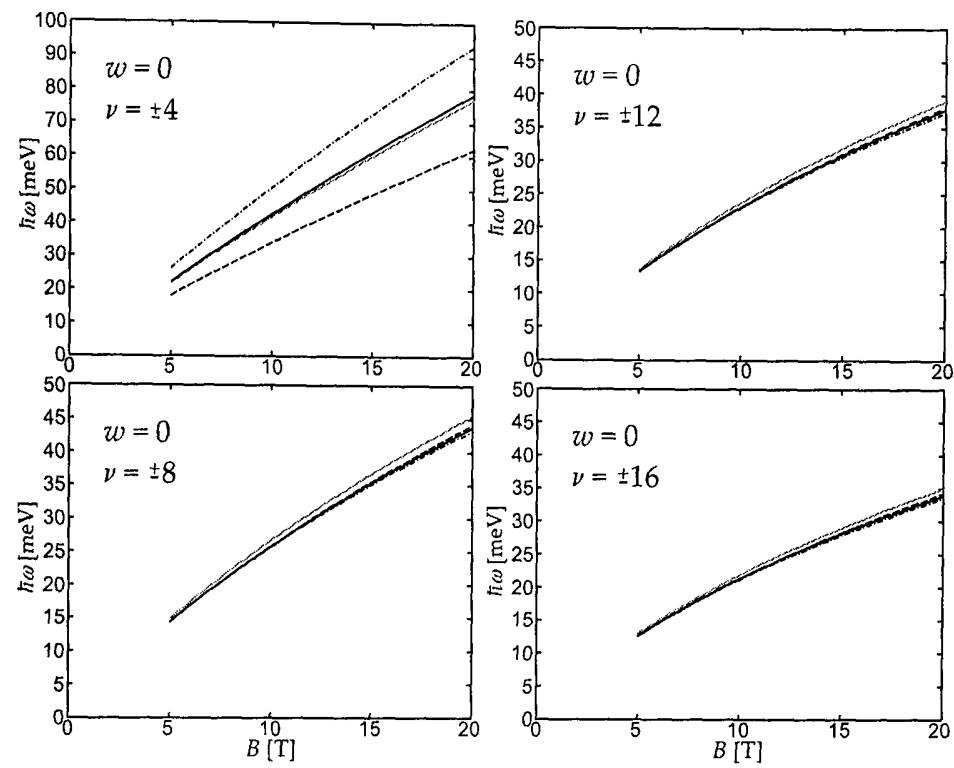

Figure 4.6: Energy of low-energy inter-LL excitations as a function of magnetic field for $w=0$. The broken lines are the contributions of individual valleys to the transition energy: black dashed and dot-dashed lines denote the transition energy for positive (negative) $v$ at $K_{+}\left(K_{-}\right)$and $K_{-}\left(K_{+}\right)$, respectively. The solid black lines show the contribution of both valleys to the transition energy, calculated according to Eq. (4.22) (in this case the transition energy is the same for both positive and negative $v$ ), whereas solid grey lines depict the transition energy in a neutral $(u=0)$ structure. Note that for $v=8,12,16$ all black lines are very close to each other and difficult to resolve.

this problem has also been adopted in [144], where the dependence of interlayer asymmetry on the density $n$ and its influence on Landau level transition energies was estimated by neglecting screening effects). As explained before, in that experiment infrared light of energy $\hbar \omega$ was incident on the graphene bilayer in a strong external magnetic field and with a constant filling factor in order to excite charge carriers between a prescribed pair of LLs and to probe the energy dispersion. According to the selection rules determined in Sec. 4.2, only transitions which change the LL index $m$ by one are allowed. Also, as photons provide a very small momentum transfer, we only consider transitions between levels at the same $K$ point. Thus, the corresponding low-energy transitions for filling factors $v=+4,+8,+12,+16$ are $1 \xi \rightarrow+2 \xi,+2 \xi \rightarrow+3 \xi,+3 \xi \rightarrow+4 \xi$, and $+4 \xi \rightarrow+5 \xi$, respectively. For filling factors $v=-4,-8,-12,-16$, they are 

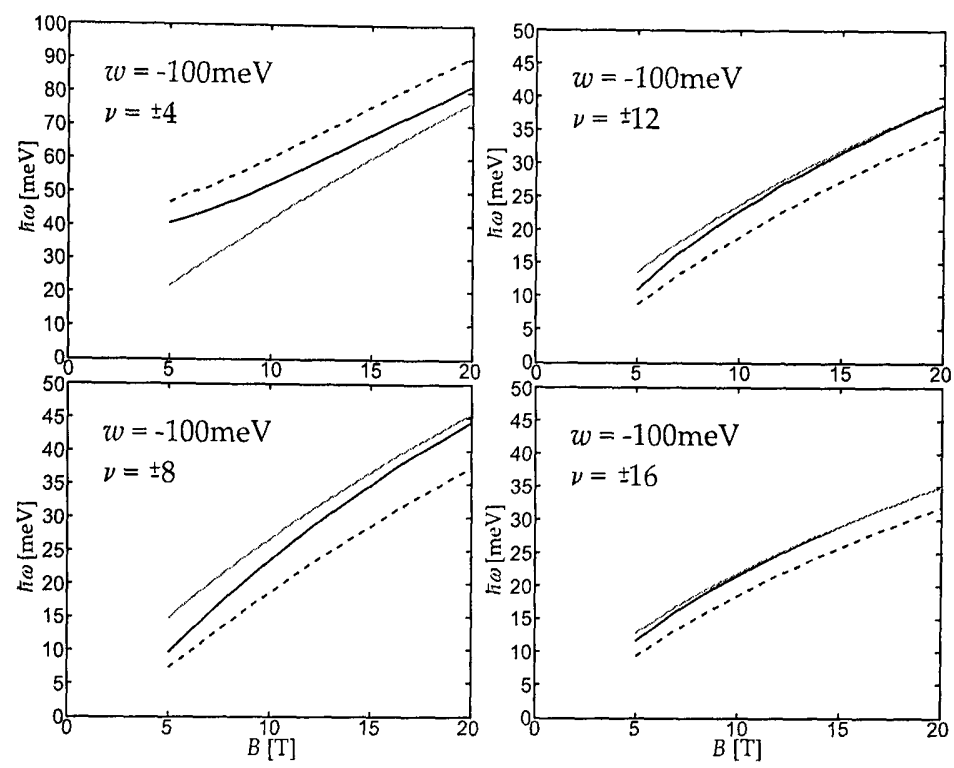

Figure 4.7: Energy of low-energy inter-LL excitations as a function of magnetic field for $w=-100 \mathrm{meV}$. Black solid and dashed lines denote the transition energy for positive and negative filling factor, respectively. Grey solid lines depict the transition energy in a neutral $(u=0)$ structure.

$-2 \xi \rightarrow 1 \xi,-3 \xi \rightarrow-2 \xi,-4 \xi \rightarrow-3 \xi$, and $-5 \xi \rightarrow-4 \xi$, respectively. However, as transitions between the same levels at different $K$ points differ too little in energy to have been resolved separately in the abovementioned experiment (in fact, they can be only be clearly distinguished in Fig. 4.6 for the case $v=4$ ), we obtain a single transition energy $\epsilon_{\text {trans }}^{v}$ for a given filling factor $\nu$ by comparing the relative intensities of the corresponding transition at each $K$ point:

$$
\epsilon_{\text {trans }}^{v}=\frac{\epsilon_{\text {trans }}^{v}\left(K_{+}\right) I^{v}\left(K_{+}\right)+\epsilon_{\text {trans }}^{v}\left(K_{-}\right) I^{v}\left(K_{-}\right)}{I^{v}\left(K_{+}\right)+I^{v}\left(K_{-}\right)}
$$

where $\epsilon_{\text {trans }}^{v}\left(K_{\xi}\right)$ and $I^{v}\left(K_{\xi}\right)$ are the transition energy at the $K_{\xi}$ point and its intensity, respectively. The results obtained for sets of parameters as in Fig. 4.5(a) and $(b)$ are shown in Fig. $4.6(w=0)$ and Fig. $4.7(w=-100 \mathrm{meV})$. We shall first discuss the case $w=0$ presented in Fig. 4.6. In this case, for a specified value of $B$, the asymmetry $u$ changes sign with a change of the sign of the filling factor [Fig. 4.5(a)], the Landau level spectrum for $v$ and $-v$ are the same but the $K$ 
points have to be exchanged. Therefore, $\epsilon_{\text {trans }}^{\nu}\left(K_{+}\right)=\epsilon_{\text {trans }}^{-\nu}\left(K_{-}\right)$, clearly seen in all four graphs. Moreover, both transitions have the same intensity and contribute equally to $\epsilon_{\text {trans }}^{v}$ (black solid line). Comparison with the transition energy for a neutral bilayer (grey line) shows that non-zero $u$ decreases the energy of the transition. The greater $|u|$ and $B$, the bigger the difference between excitation energy in symmetric and biased bilayers. However, this difference decreases with an increase of filling factor.

Introduction of parameter $w$ breaks symmetry between the conduction and valence band LLs as presented in Fig. 4.7 for the case of $w=-100 \mathrm{meV}$. The valence band excitation has greater energy than the conduction band excitation at filling factor $v= \pm 4$. However, this situation is reversed for higher filling factors $v= \pm 8, \pm 12, \pm 16$ (this reversal was not observed in the experiment [63]). For this specific case, $w=-100 \mathrm{meV}$, the asymmetry introduced between excitations for filling factors $v$ and $-v$ is of the size of $3-10 \mathrm{meV}$. These two effects, the reduction of the transition energy with the increase of $u$ and the breaking of the symmetry between transitions for positive and negative filling factor caused by $w$, partly account for the disagreement between experimental findings and Eq. (4.5) obtained from a tight-binding model for neutral bilayers as used in Ref. [63] to fit the data. Other investigations [145-147] show that additional corrections may arise from electron-electron interactions.

\subsubsection{Magneto-optical spectra in charged bilayer: high-energy inter-Landau level transitions}

Using the self-consistently obtained values of the interlayer asymmetry $u$ for a given filling factor $v$ and magnetic field $B$, we can analyse again the optical transition spectra corresponding to transitions between LLs in split bands of the bilayers, Sec. 4.2. With the help of Eq. (4.19) describing the intensity of absorption in external magnetic field, we compute the infrared optical absorp- 


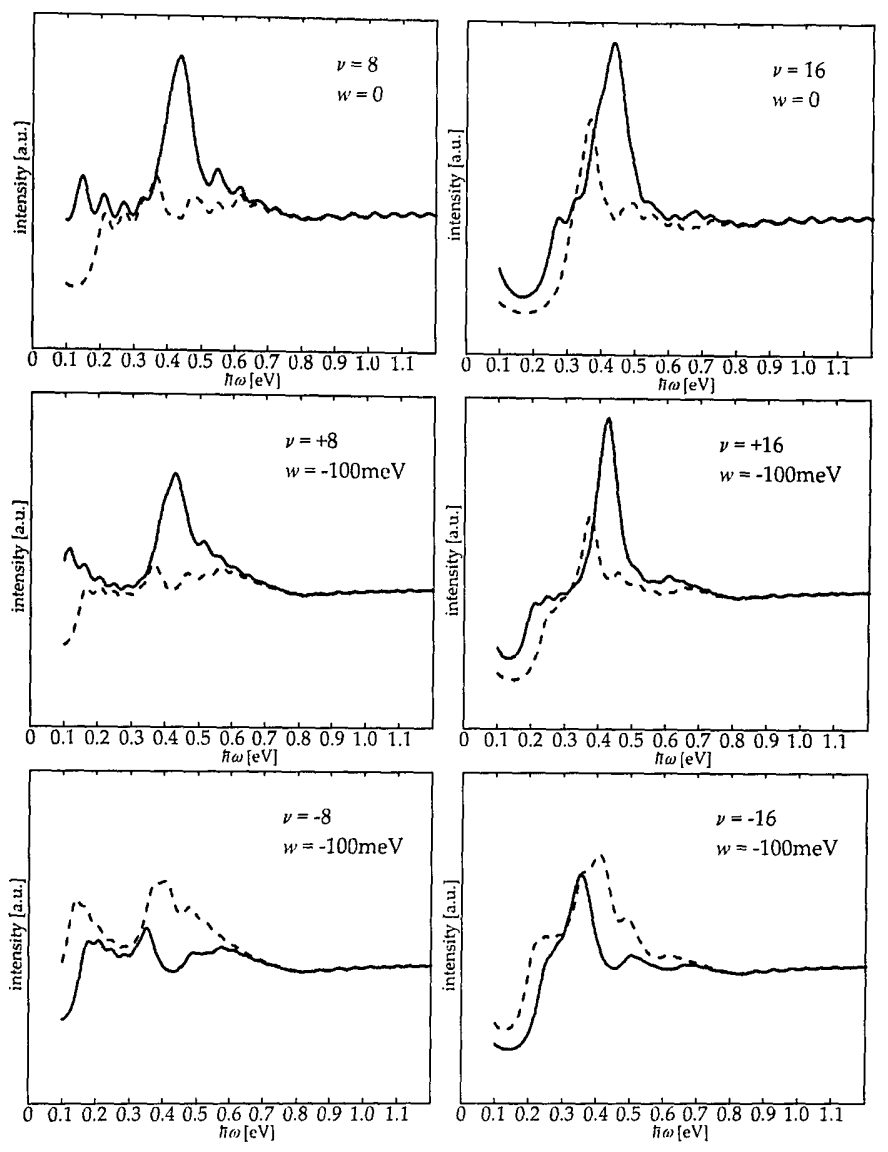

Figure 4.8: Magneto-optical absorption spectra of bilayer graphene in strong external magnetic field $B=14 \mathrm{~T}$ and for filling factors $v=8$ and $v=16$ (left and right columns, respectively) and the case of $w=0$ (top row) and $w=$ $-100 \mathrm{meV}$ (middle and bottom row). For the symmetric case of $w=0$, solid and dashed lines show absorption of right-handed (left-handed) and left-handed (right-handed) circularly polarised light for the positive (negative) filling factor, respectively. For the case of $w=-100 \mathrm{meV}$, solid and dashed lines represent absorption of right and left-handed circularly polarised light, respectively.

tion spectra of right $(\oplus)$ and left-handed $(\Theta)$ circularly polarized light for bilayer graphene in a strong external magnetic field. As opposed to Fig. 4.3(c), we describe this time a charged bilayer with significant interlayer asymmetry. The broadening of the Landau levels is again modeled using a Lorentzian shape with the same full width at half maximum $\gamma=60 \mathrm{meV}$ for all Landau levels. Numerical results for magnetic field $B=14 \mathrm{~T}$ and filling factors $v=8$ and $v=16$ are shown in Fig. 4.8. For the case of $w=0$, the symmetry of the system demands that the intensity of absorption of light with a given polarisation for 
filling factor $v$ and is the same as that of the light with the inverted polarisation for filling factor $-v$. This, indeed, is the case for graphs in the top row of Fig. 4.8, where black solid and dashed lines show absorption of right-handed (left-handed) and left-handed (right-handed) circularly polarised light for the positive (negative) filling factor, respectively. Such a symmetry is broken for the case of $w=-100 \mathrm{meV}$, for which the spectra for positive and negative filling factors are shown in the separate panels, where solid and dashed lines refer to right-handed and left-handed polarisation, respectively. In particular, the peak visible for some of the spectra at the radiation energy around $0.4 \mathrm{eV}$ corresponds to electron excitation between the low-energy $n=0 \mathrm{LL}$ and one of the two high-energy $n=1$ LLs. Its position can be used to determine the value of the coupling constant $\gamma_{1}$, and a small shift in energy of this peak is due to strong magnetic field and asymmetry $u$. 


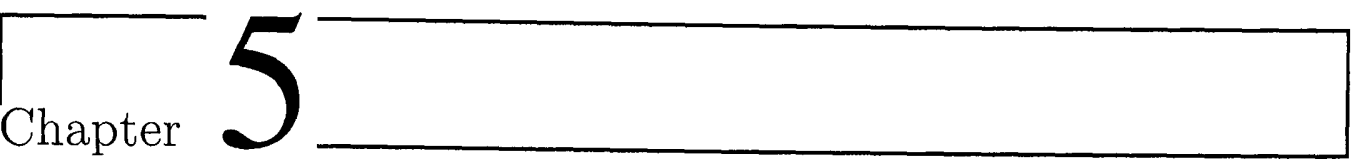

\section{Electronic Raman Spectroscopy}

As discussed in Sec. 4.3, experimental measurements of the bilayer graphene Landau level structure with infrared absorption showed that tight-binding description for neutral bilayer is unable to describe all the important physics [63]. Some theoretical explanations were suggested [73, 145-147], but the issue has not yet been clarified. It would be therefore beneficial to have at one's disposal another probe of the Landau level structure but with different selection rules. Then, electronic excitations between different pairs of levels would be measured. This could help gain more insight into the physics of the problem. In this chapter, we investigate the possibility of using electronic Raman spectroscopy as such a probe.

The electronic Raman spectroscopy can provide information about various single particle and collective electron excitations in the system studied. In semiconductors, it has been, for example, employed to investigate donor and acceptor states, plasmons and spin-density fluctuations involving electron spin-flip due to the spin-orbit interaction [74, 148]. The inelastic scattering of photons on electrons in semiconductor placed in an external magnetic field was first discussed by Wolff, who pointed out that unequal spacing of the LLs resulting from nonparabolicity of the electronic bands is crucial for the electron-photon interaction matrix elements not to vanish [149]. The features corresponding to the electronic contribution to the Raman scattering in an external magnetic 
field were observed in many semiconductors, for example, InSb [150] and GaAs [151]. Very recently, the Raman spectroscopy of electronic excitations in monolayer graphene has been investigated theoretically [152]. It has been shown that at high magnetic fields the inelastic light scattering accompanied by the excitation of the electronic mode with the highest quantum efficiency involves the generation of inter-band electron-hole pairs. At high (quantizing) magnetic fields this leads to the electron excitations from the Landau level $n^{-}$(with energy $\left.\epsilon_{n^{-}}=-\sqrt{2 n} \hbar v / \lambda_{B}\right)$ in the valence band to the Landau level $n^{+}\left(\epsilon_{n^{+}}=\sqrt{2 n} \hbar v / \lambda_{B}\right)$ in the conduction band with energies $\omega_{n}=2 \sqrt{2 n} \hbar v / \lambda_{B}$ and crossed polarisation of in/out photons. This fact, contrasted with the $\Delta n= \pm 1$ selection rules for transitions between Landau levels in the absorption of left and right-handed circularly polarised infrared photons [69], is the first hint that electronic Raman spectroscopy provides data supplementary to that obtained in optical absorption.

Here, we investigate the contribution of the low-energy electronic excitations towards the Raman spectrum of bilayer graphene for the incoming photon energy $\Omega \gtrsim 1 \mathrm{eV}$. Starting with the four-band tight-binding model, we derive an effective scattering amplitude that can be incorporated into the two-band approximation. We show that due to the influence of the high-energy bands, this effective scattering amplitude is different from the contact interaction amplitude obtained within the two-band model alone. We then calculate the spectral density of the inelastic light scattering accompanied by the excitation of electronhole pairs in bilayer graphene. In the absence of a magnetic field, due to the parabolic dispersion of the low-energy bands in a bilayer crystal, this contribution is constant and in doped structures has a threshold at twice the Fermi energy. In an external magnetic field, the dominant Raman-active modes are the $n^{-} \rightarrow n^{+}$inter-Landau-level transitions with crossed polarisation of in/out photons. Finally, our estimate of the quantum efficiency of a single $n^{-} \rightarrow n^{+}$ 
transition peak in the magnetic field of 10T, $I_{n^{-} \rightarrow n^{+}} \sim 10^{-12}$, suggests that these features may be observable experimentally [75].

\subsection{The two-photon field and the electron-photon in- teraction}

As a starting point we choose the tight-binding Hamiltonian in the linear approximation, eq. (2.9). We shall keep terms up to quadratic in the electronic momentum $p$, but disregard the least important couplings, $v_{4}$ and $\gamma_{n}$. Then, using the set of Pauli matrices, $\sigma_{x}, \sigma_{y}$ and $\sigma_{z}$, the Hamiltonian $\hat{\boldsymbol{H}}_{\xi}(\boldsymbol{p})$ describing a single electron with momentum $p$ in the vicinity of the valley $K_{\xi}$ can be written in the form

$$
\begin{aligned}
\hat{\boldsymbol{H}}_{\xi}(\boldsymbol{p}) & =\xi\left(\begin{array}{cc}
v_{3}(\boldsymbol{\sigma} \cdot \boldsymbol{p})^{T} & v \sigma \cdot \boldsymbol{p} \\
v \sigma \cdot \boldsymbol{p} & \xi \gamma_{1} \sigma_{x}
\end{array}\right) \\
& -\mu\left(\begin{array}{cc}
\frac{v_{3}}{v}\left[\sigma_{x}\left(p_{x}^{2}-p_{y}^{2}\right)+2 \sigma_{y} p_{x} p_{y}\right] & \sigma_{x}\left(p_{x}^{2}-p_{y}^{2}\right)-2 \sigma_{y} p_{x} p_{y} \\
\sigma_{x}\left(p_{x}^{2}-p_{y}^{2}\right)-2 \sigma_{y} p_{x} p_{y} & 0
\end{array}\right) .
\end{aligned}
$$

To describe the process of inelastic scattering of light on electrons in our material, we consider an experimental setup in which incoming laser light of energy $\Omega \gg \gamma_{1}$, in-plane momentum $\boldsymbol{q}$ (out-of-plane component of momentum equal to $\left.q_{z}=\sqrt{\Omega^{2} / c^{2}-q^{2}}\right)$ and polarisation $l$ is shined onto to the sample. Scattered photon has polarisation $\tilde{l}$, in-plane momentum $\tilde{\boldsymbol{q}}$ and energy $\tilde{\Omega}=\Omega-\omega$, where $\omega$ is the Raman shift. We also assume the temperature $T$ to be smaller than the Raman shift, $k_{B} T<\omega$ ( $k_{B}$ is the Boltzmann's constant). In our case, the inelastic light scattering may occur via (I) a one-step process [so called contact interaction, Fig. 5.1(a)] or (II) a two-step process involving an intermediate state [Fig. 5.1(b)]. The two-step process involves: the absorption (or emission) of a photon with energy $\Omega(\tilde{\Omega})$ transferring an electron with momentum $p$ from an 


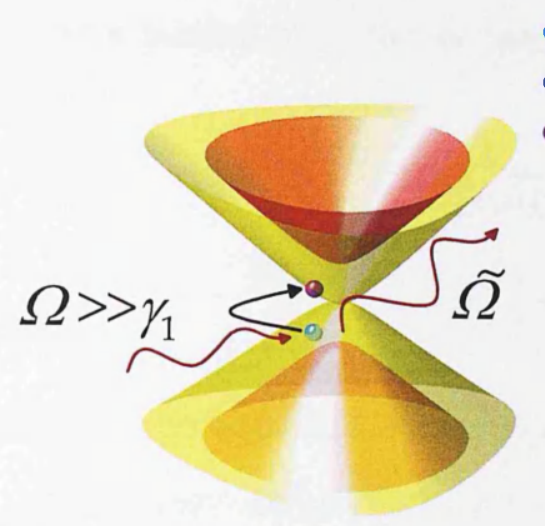

(a) one-step process
- initial state

- intermediate state

- final state

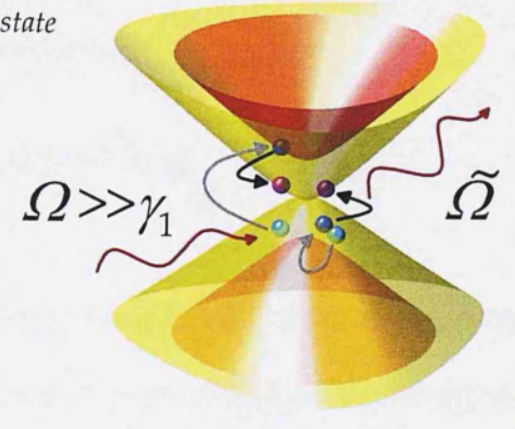

(b) two-step processes

Figure 5.1: Schematic depiction of the (a) one and (b) two-step ERS processes considered in this text, shown for the valley $K_{+}$. For the two-step processes, grey (black) solid lines indicate the first (second) step of the process. Also, in (b), two different cases of the possible two-step process, one involving an intermediate state in the high-energy band (sequence with the dark blue ball on the red high-energy band) and one only involving states in the low-energy bands, have been shown. The light blue (purple) circle denotes the hole (electron) in the final electron-hole pair, while the dark blue circle represents the intermediate virtual state (if relevant). Note that for any intermediate state $|v\rangle$ with energy $\epsilon_{v}, \Omega, \tilde{\Omega} \gg \epsilon_{v}$.

occupied state in the valence band into a virtual intermediate state (energy is not conserved at this stage), followed by another electron emission (or absorption) of the second photon with energy $\tilde{\Omega}(\Omega)$. The one-step process is the usual inelastic scattering of an incoming photon on an electron with transfer of energy to the latter. As a result of both one and two-step processes, an electron-hole pair in the low-energy bands is created with the electron and the hole having almost the same momentum ( $p+q-\tilde{q}$ and $p$, respectively), since $q, \tilde{q} \ll p$ and the momentum transfer from light is negligible $\left(v / c \sim 3 \cdot 10^{-3}\right)$. Therefore, $p+q-\tilde{q} \approx p$ and due to the approximately electron-hole symmetric band structure in the vicinity of Brillouin zone corners, the electron initial and final energies $\epsilon_{i}$ and $\epsilon_{f}$ are related, $\epsilon_{f} \approx-\epsilon_{i}$.

To include the interaction of the electrons with photons, we construct the canonical momentum $\left[p-e\left(A\left(r, t^{\prime}\right)+\tilde{A}\left(r, t^{\prime \prime}\right)\right)\right]$, where $A\left(r, t^{\prime}\right)$ and $\tilde{\boldsymbol{A}}\left(r, t^{\prime \prime}\right)$ are 
the vector potentials of the incoming and outgoing light, respectively,

$$
\begin{aligned}
& \boldsymbol{A}\left(r, t^{\prime}\right)=\frac{1}{\sqrt{2 \epsilon_{0} V \Omega}}\left(l e^{i\left(q \cdot r-\Omega t^{\prime}\right) / \hbar} b_{q, q_{z}, l}+\text { H.c }\right) ; \\
& \tilde{A}\left(r, t^{\prime \prime}\right)=\frac{1}{\sqrt{2 V \epsilon_{0} \tilde{\Omega}}}\left(\tilde{l}^{*} e^{-i\left(\tilde{q} \cdot r-\tilde{\Omega} t^{\prime \prime}\right) / \hbar} b_{\tilde{q}, \tilde{q}_{z}, \tilde{l}}^{+}+\text {H.c. }\right),
\end{aligned}
$$

$V$ denotes the normalisation volume appropriate for the incoming photons and $b_{\boldsymbol{q}, q_{z}, l}$ is an annihilation operator for a photon with in-plane momentum $\boldsymbol{q}$, outof-plane momentum component $q_{z}$ and polarisation $l$. We then expand the Hamiltonian $\hat{\boldsymbol{H}}_{\xi}(p-e[\boldsymbol{A}+\tilde{\boldsymbol{A}}])$ up to the second order in the vector potential and write down the interaction part,

$$
\hat{\boldsymbol{H}}_{\mathrm{int}}=\hat{j} \cdot\left(\boldsymbol{A}\left(\boldsymbol{r}, t^{\prime}\right)+\tilde{\boldsymbol{A}}\left(r, t^{\prime \prime}\right)\right)+\frac{e^{2}}{2} \sum_{i, j} \frac{\partial^{2} \hat{\boldsymbol{H}}_{\xi}}{\partial p_{i} \partial p_{j}} A_{i} \tilde{A}_{j}
$$

where $\hat{j}=-e \frac{\partial \hat{\boldsymbol{H}}_{\xi}}{\partial p}$ is the current vertex.

\subsection{Theory of Raman scattering in graphene}

To describe the electronic contribution to Raman scattering in bilayer graphene, we aim to calculate the spectral density $g(\omega)$ and the quantum efficiency (intensity) of the Raman scattering, I. The quantum efficiency describes the ratio of the flux of outgoing, inelastically scattered photons to the flux of the incoming photons, and is an integral, $I=\int d \omega g(\omega)$, of the spectral density $g(\omega)$ representing the probability for the incoming photon to scatter inelastically with energy $\tilde{\Omega}=\Omega-\omega$, where $\omega$ is the Raman shift. Hence, the quantum efficiency expresses the total probability for single incoming photon to scatter inelastically on an electron and excite an electron-hole pair in the low-energy bands. The probability for the incoming photon to scatter with the Raman shift $\omega$ in a particular direction (defined by the momentum $\tilde{\boldsymbol{q}}$ of the scattered photon), is, in turn, characterised by the angle-resolved probability of scattering $w(\tilde{q})$. Finally, the 
scattering probability $w(\tilde{\boldsymbol{q}})$ that one photon is scattered with the excitation of an electron-hole (e-h) pair in the final state is related, as $w \propto|\overline{\mathcal{R}}|^{2}$, to the scattering amplitude $\overline{\mathcal{R}}$ of the Raman process. This scattering amplitude $\overline{\mathcal{R}}$ is a sum of two contributions: $\mathscr{\mathcal { R }}$ due to the one-step processes (contact interaction) and $\mathcal{R}$ due to the two-step processes.

\subsubsection{Contribution of the contact interaction}

The contact interaction scattering amplitude $\mathcal{R}$ results from the second term in the interaction Hamiltonian (5.2). It is characterised by operators $\partial^{2} \hat{H} / \partial p_{i} \partial p_{j}$ and hence, the first term in the Hamiltonian in (5.1) does not allow any contact interaction processes, as it includes only terms linear in the electron momentum $p$. The only contribution to the contact interaction comes from the second term in the right-hand side of (5.1), which contains terms quadratic in $p$. Such a contribution involves prefactor $\frac{v^{2}}{6 \gamma_{0} \Omega}$ determining the smallness of the amplitude $\delta R$ of one-step Raman scattering of photons with energy less than the band-width of graphene, $\sim 6 \gamma_{0}$. Detailed calculations yield the contribution to the scattering amplitude due to the contact interaction obtained within the four-band model,

$$
\begin{aligned}
& \delta R=\frac{e^{2} \hbar^{2} v^{2}}{6 \epsilon_{0} V \gamma_{0} \sqrt{\Omega \tilde{\Omega}}} \mathcal{L} \cdot \boldsymbol{d} ; \\
& \boldsymbol{d}=\left(l_{x} \tilde{l}_{y}^{*}+l_{y} \tilde{i}_{x}^{*} l_{x} \tilde{l}_{x}^{*}-l_{y} \tilde{l}_{y}^{*}\right) ; \quad \mathcal{L}=\left(\mathcal{L}_{x}, \mathcal{L}_{y}\right) ; \\
& \mathcal{L}_{x}=\left(\begin{array}{cc}
-\frac{v_{3}}{v} \sigma_{y} & \sigma_{y} \\
\sigma_{y} & 0
\end{array}\right) ; \mathcal{L}_{y}=\left(\begin{array}{cc}
-\frac{v_{3}}{v} \sigma_{x} & -\sigma_{x} \\
-\sigma_{x} & 0
\end{array}\right) .
\end{aligned}
$$




\subsubsection{Contribution of the two-step processes}

To find $\mathcal{R}$, we describe a two-step transition which involves an intermediate virtual state $|v\rangle$ with energy $\epsilon_{v}$, as

$$
\begin{aligned}
& \mathcal{R}=\frac{-1}{2 \epsilon_{0} V \sqrt{\Omega \tilde{\Omega}}} \\
& \times\left\{\sum_{\nu} \int_{-\infty}^{\infty} \int_{-\infty}^{t^{\prime}} e^{\frac{i}{\hbar}\left(\epsilon_{f}-\epsilon_{\nu}\right) t^{\prime}}\left(j \cdot \tilde{l}^{*}\right) e^{\frac{-i}{\hbar}\left(\tilde{q} \cdot r-\tilde{\Omega^{\prime}}\right)}|v\rangle\langle v| e^{\frac{i}{\hbar}\left(q \cdot r-\Omega t^{\prime \prime}\right)}(j \cdot l) e^{\frac{i}{\hbar}\left(\epsilon_{\nu}-\epsilon_{i}\right) t^{\prime \prime}} d t^{\prime} d t^{\prime \prime}\right. \\
& \left.\left.+\sum_{\nu} \int_{-\infty}^{\infty} \int_{-\infty}^{t^{\prime}} e^{\frac{i}{\hbar}\left(\epsilon_{f}-\epsilon_{\nu}\right) t^{\prime}}(j \cdot l) e^{\frac{i}{\hbar}\left(q \cdot r-\Omega t^{\prime}\right.}|v\rangle\langle v| e^{\frac{-i}{\hbar}\left(\tilde{q} \cdot r-\Omega t^{\prime \prime}\right.}\right)\left(j \cdot \tilde{l}^{\tilde{x}}\right) e^{\frac{i}{\hbar}\left(\epsilon_{\nu}-\epsilon_{i}\right) t^{\prime \prime}} d t^{\prime} d t^{\prime \prime}\right\} .
\end{aligned}
$$

The virtual state $|v\rangle$ may belong to any of the four bands, since an electron is excited from a state with momentum $p$ to a state with momentum $p+q$ or $p-\tilde{q}$ depending on the accompanying photon process. In Eq. (5.4), the first (second) term corresponds to processes in which the photon is absorbed (emitted) in the first step and emitted (absorbed) in the second step of the process. Integration over time in those expressions can be performed by changing variables to $\tau=$ $t^{\prime}-t^{\prime \prime}$, which varies at the scale of $\omega^{-1}, \omega=\Omega-\tilde{\Omega}$, and $\bar{t}=\left(t^{\prime}+t^{\prime \prime}\right) / 2$, which varies at the scale of $\bar{\Omega}^{-1}, \bar{\Omega}=\left(\Omega+\Omega^{\prime}\right) / 2 \gg \omega$. For incoming and outgoing photons, $\Omega, \tilde{\Omega} \gg \gamma_{1}$. We also concentrate here on the low-energy excitations in the final states with $\omega \ll \gamma_{1}$. This allows us to expand factors $\frac{1}{ \pm \bar{\Omega}-\varepsilon_{v}}$ resulting from the integration over $\tau$ in powers of $\left(\epsilon_{v} / \Omega\right)$, keeping terms of the order of 1 and $\left(\gamma_{1} / \Omega\right)$ (the latter appear when the virtual state is taken to be in the highenergy bands) and to perform summation over the intermediate virtual states 
of the process. Consequently, the amplitude $\mathcal{R}$ takes the form of a matrix

$$
\begin{gathered}
\mathcal{R} \approx \frac{e^{2} \hbar^{2} v^{2}}{\epsilon_{0} V \Omega \sqrt{\Omega \tilde{\Omega}}}\left\{-i\left(\begin{array}{cc}
\sigma_{z} & 0 \\
0 & \sigma_{z}
\end{array}\right)\left(l \times \tilde{l}^{*}\right)_{z}+\frac{1}{\Omega} \mathcal{M} \cdot \boldsymbol{d}\right\} \delta\left(\epsilon_{f}-\epsilon_{i}-\omega\right), \\
\mathcal{M}=\left(\mathcal{M}_{x}, \mathcal{M}_{y}\right), \\
\mathcal{M}_{x}=\left(\begin{array}{cc}
\gamma_{1} \sigma_{y} & \xi v\left(\sigma_{y} p_{x}+\sigma_{x} p_{y}\right) \\
\xi v\left(\sigma_{y} p_{x}+\sigma_{x} p_{y}\right) & 0
\end{array}\right), \\
\mathcal{M}_{y}=\left(\begin{array}{cc}
\gamma_{1} \sigma_{x} & \xi v\left(\sigma_{x} p_{x}-\sigma_{y} p_{y}\right) \\
\xi v\left(\sigma_{x} p_{x}-\sigma_{y} p_{y}\right) & 0
\end{array}\right) .
\end{gathered}
$$

\subsubsection{The resulting Raman scattering amplitude}

The scattering amplitude $\overline{\mathcal{R}}$ of the Raman process acompanied by electronhole excitation is a sum of $\mathcal{R}$ and $\delta \mathcal{R}$. From the comparison of the corresponding prefactors $\frac{e^{2} \hbar^{2} v^{2}}{6 \epsilon_{0} \gamma_{0} \sqrt{\Omega \tilde{\Omega}}}$, Eq. 5.3, and $\frac{e^{2} \hbar^{2} v^{2}}{\epsilon_{0} \Omega} \sqrt{\Omega \tilde{\Omega}}$, Eq. 5.5, it follows that $\delta \mathcal{R} \ll \mathcal{R}$, as $6 \gamma_{0} \gg \Omega$. Hence, we can neglect the contribution of the one-step processes,

$$
\overline{\mathcal{R}}=\mathcal{R}+\delta \mathcal{R} \approx \mathcal{R} .
$$

Also, we are mostly interested in the low-energy properties of our material. To analyse the contribution of electronic modes toward the low-energy part of Raman spectrum with the photon energy shift $\omega<\gamma_{1} / 2$, which is determined by the excitation of the electron-hole pairs in the low-energy bands with $v p \ll \gamma_{1}$, we use the effective low-energy Hamiltonian, Eq. 2.12 in Sec. 2.3,

$$
\hat{H}_{\mathrm{eff}}=-\frac{v^{2}}{\gamma_{1}}\left[\left(p_{x}^{2}-p_{y}^{2}\right) \sigma_{x}+2 p_{x} p_{y} \sigma_{y}\right]
$$

To characterise the excitation of the low-energy modes corresponding to the 
transitions between low-energy band states described by $\hat{\boldsymbol{H}}_{\text {eff, we take only the }}$ part of $\mathcal{R}$ which acts in that two-dimensional Hilbert space, keep terms in the lowest relevant order in $v p / \gamma_{1} \ll 1$ and $\gamma_{1} / \Omega \ll 1$, and write down an effective amplitude $\mathcal{R}_{\text {eff }}$

$$
\mathcal{R}_{\mathrm{eff}} \approx \frac{e^{2} \hbar^{2} v^{2}}{\epsilon_{0} V \Omega \sqrt{\Omega \tilde{\Omega}}}\left\{-i \sigma_{z}\left(l \times \tilde{l}^{*}\right)_{z}+\frac{\gamma_{1}}{\Omega}\left[\sigma_{x} d_{y}+\sigma_{y} d_{x}\right]\right\}
$$

We point out that the above matrix cannot be obtained within a theory constrained by the two-band approximation from the very beginning. Seemingly, one may try to define a contact-interaction-like term due to the terms quadratic in the electron momentum $p$ in the Hamiltonian in Eq. (5.7), which carries a prefactor $\frac{e^{2} \hbar^{2} v^{2}}{\epsilon_{0} \gamma_{1} \Omega}$. Such a prefactor may suggest a greater magnitude of scattering than prefactor $\frac{e^{2} \hbar^{2} v^{2}}{\epsilon_{0} \Omega^{2}}$ in the amplitude $\mathcal{R}_{\text {eff }}$ above. However, the scattering amplitude obtained within the two-band model can only be applied to photons with $\Omega<\gamma_{1}$, which is hardly relevant for Raman spectroscopy since the latter is usually performed with laser beams using $\Omega \sim 1.3$ to $2.8 \mathrm{eV}$ [89-92, 153-157].

\subsection{ERS spectra in the absence of the magnetic field}

To calculate the angle-resolved probability of the Raman scattering, $w(\tilde{\boldsymbol{q}})$, we consider the ratio of the energy flux of the outgoing photons scattered into a state with momentum $\tilde{q}, \phi_{\tilde{q}}^{\text {out }}$, to the energy flux of the incoming photons with momentum $q, \phi_{q}^{\text {in }}$

$$
w(\tilde{\boldsymbol{q}})=\frac{\phi_{\tilde{q}}^{\text {out }}}{\phi_{q}^{\text {in }}} .
$$

The energy flux of the incoming photons is given by the number of photons with momentum $\boldsymbol{q}, n_{q}$, found in volume $V$, each carrying energy $\Omega, \phi_{q}^{\text {in }}=\frac{\Omega n_{q}}{V}$. The energy flux of the outgoing photons is described by the number of photons scattered in unit time, $\dot{n}_{\tilde{q}}$, and originating from an area $S$ of the material, their 
energy $\tilde{\Omega}$ and the speed of light, $c$, as $\phi_{\tilde{q}}^{\text {out }}=\frac{\tilde{\Omega} \dot{n}_{\tilde{q}}}{S c}$. We assume that the only processes creating the outgoing photons in the state with momentum $\tilde{q}$ are those under consideration here and therefore,

$$
\dot{n}_{\tilde{q}}=\frac{S}{2 \pi \hbar^{3}} \int d p|\overline{\mathcal{R}}|^{2} n_{q}
$$

where we used Fermi's golden rule to include the scattering probability and integrated out the electron states. Using Eq. (5.9) and the above relations, the angle-resolved probability of the Raman scattering, $w(\tilde{\boldsymbol{q}} \approx 0)$, is

$$
w=\frac{2 V \tilde{\Omega}}{c \pi \hbar^{3} \Omega} \int d p\left|\left\langle f\left|\mathcal{R}_{\mathrm{eff}}\right| i\right\rangle\right|^{2} \times f_{i}\left(1-f_{f}\right) \delta\left(\epsilon_{i}+\omega-\epsilon_{f}\right),
$$

where $f_{i}$ and $f_{f}$ are filling factors of the initial and final electronic state, respectively, and the spin and valley degeneracies have already been taken into account. This gives ${ }^{1}$

$$
\begin{aligned}
& w \approx \frac{\gamma_{1} e^{4} \hbar v^{2}}{c \epsilon_{0}^{2} V \Omega^{4}}\left\{\Xi_{s}+\frac{\gamma_{1}^{2}}{2 \Omega^{2}} \Xi_{o}\right\} \theta(\omega-2 \mu), \\
& \Xi_{s}=\left|l \times \tilde{l}^{*}\right|^{2}, \quad \Xi_{0}=1+\left(l \times l^{*}\right) \cdot\left(\tilde{l} \times \tilde{l}^{*}\right) .
\end{aligned}
$$

Above, the first term with polarization factor $\Xi_{s}$ describes the contribution of photons scattered with the same circular polarization as the incoming beam. The second term, with polarization factor $\Xi_{0}$, represents the scattered photons with circular polarization opposite to the incoming beam.

\footnotetext{
${ }^{1}$ In the integration over the electronic momentum $p$ we neglected the trigonal warping of the electronic dispersion caused by $v_{3}$, Sec. 2.3. This is only important for very low energies. The density of states, apart from the vicinity of the Lifshitz transition, remains almost unaffected.
} 
In turn, the angle-integrated spectral density of Raman scattering $g(\omega)$ is

$$
\begin{aligned}
g(\omega) & =V \iint \frac{d \tilde{q} d \tilde{q}_{z}}{(2 \pi \hbar)^{3}} w \delta\left(\tilde{\Omega}-c \sqrt{\tilde{q}^{2}+\tilde{q}_{z}^{2}}\right) \\
& =2\left(\frac{e^{2}}{4 \pi \epsilon_{0} \hbar c} \frac{v}{c}\right)^{2} \frac{\gamma_{1}}{\Omega^{2}}\left\{2 \Xi_{s}+\frac{\gamma_{1}^{2}}{\Omega^{2}} \Xi_{o}\right\} \theta(\omega-2 \mu) .
\end{aligned}
$$

Here, the constant spectral density $g$ as a function of $\omega$ reflects the parabolicity of the low-energy bands and thus, energy-independent density of states in the bilayer. This is different in monolayer graphene, where $g(\omega) \propto \omega$, reflecting the energy-dependent density of states of electron-hole pairs [152]. The characteristic of monolayer graphene crossed polarisation of in/out photons is retained in the case of the bilayer system.

Experimentally, constant spectral density $g$ in undoped bilayer graphene is impossible to distinguish from a homogeneous background. However, if the chemical potential $\mu$ is not at the neutrality point, then transitions with $\omega<2 \mu$ are essentially blocked. Although new processes, resulting in the creation of the intraband electron-hole pair excitations and very small $\omega$, are possible for $\mu \neq 0$, their contribution carries additional prefactor $v / c \sim \frac{1}{300}$ [149]. Explicit calculation performed for the monolayer graphene showed that the quantum efficiency of the intraband transitions was of the order of $10^{-15}$ [152]. In contrast, for chemical potential $\mu \sim 50 \mathrm{meV}$ (corresponding to additional carrier density $n_{0} \sim 1.5 \times 10^{12} \mathrm{~cm}^{-2}$ ), the lost quantum efficiency due to the blocked interband transitions is, according to eq. (5.11), $\Delta I \sim 10^{-12}$.

\subsection{ERS spectra in quantizing magnetic fields}

The quantization of electron states into Landau levels gives the Raman spectrum due to the electronic excitations, a pronounced structure which can be used to detect their contribution experimentally. We only consider here low-energy Landau levels, as at high energies the Landau level broadening due to, for ex- 
ample, electron-phonon interaction, will smear out the LL spectrum. In strong magnetic fields, low-energy Landau levels are sufficiently described [70] by

$$
\begin{aligned}
& \epsilon_{n^{\alpha}}=\alpha \frac{2 \hbar^{2} v^{2}}{\gamma_{1} \lambda_{B}^{2}} \sqrt{n(n-1)} \\
& \Psi_{n^{\alpha}}=\left(\begin{array}{c}
\psi_{n} \\
0
\end{array}\right) \text { for } n=0,1, \quad \Psi_{n^{\alpha}}=\frac{1}{\sqrt{2}}\left(\begin{array}{c}
\psi_{n} \\
\alpha \psi_{n-2}
\end{array}\right) \text { for } n \geq 2,
\end{aligned}
$$

where $\lambda_{B}=\sqrt{\hbar / e B}$ is the magnetic length, $n$ is the Landau level index and $\alpha=+$ denotes the conduction and $\alpha=-$ the valence band. Also, $\psi_{n}$ is the normalised $n$-th Landau level wavefunction. In a neutral bilayer, all LLs have additional fourfold degeneracy (two due to the electron spin and two due to the valley). Moreover, levels $n=0$ and $n=1$ are degenerate at $\epsilon=0$ giving rise to an 8-fold degenerate LL. We can project our effective transition amplitude $\mathcal{R}_{\text {eff }}$ onto the eigenstates $\Psi_{n^{\alpha}}$ to find the electronic Raman spectrum in the presence of a strong external magnetic field. This leads to the following selection rules for allowed electronic transitions from the initial level $n^{-}$:

$$
\text { (i) } n^{-} \rightarrow n^{+} ; \quad \text { (ii) }(n \mp 1)^{-} \rightarrow(n \pm 1)^{+} \text {. }
$$

Among those, (i) is the dominant transition. These selection rules, represented schematically in Fig. 5.2, show that using Raman spectroscopy, one can probe different electronic excitations than in optical spectroscopy, where the selection rules are $\Delta n= \pm 1[69,71]$. For a neutral bilayer, the angle-integrated spectral density $g(\omega)$ of Raman scattering in the magnetic field is equal to

$$
\begin{aligned}
& g(\omega) \approx 16 \Xi_{s}\left(\frac{e^{2}}{4 \pi \epsilon_{0} \hbar c} \frac{v}{c}\right)^{2}\left(\frac{\hbar v}{\lambda_{B} \Omega}\right)^{2} \sum_{n \geq 2} \gamma\left(\omega-2 \epsilon_{n^{+}}\right)+\delta g(\omega) \\
& \delta g(\omega)=8 \Xi_{0}\left(\frac{\gamma_{1}}{\Omega}\right)^{2}\left(\frac{e^{2}}{4 \pi \epsilon_{0} \hbar c} \frac{v}{c}\right)^{2}\left(\frac{\hbar v}{\lambda_{B} \Omega}\right)^{2}\left[\sum_{n=1,2} 2 \gamma\left(\omega-\epsilon_{(n+1)^{+}}\right)+\sum_{n \geq 3} \gamma\left(\omega-\epsilon_{(n+1)^{+}}-\epsilon_{(n-1)^{+}}\right)\right] .
\end{aligned}
$$



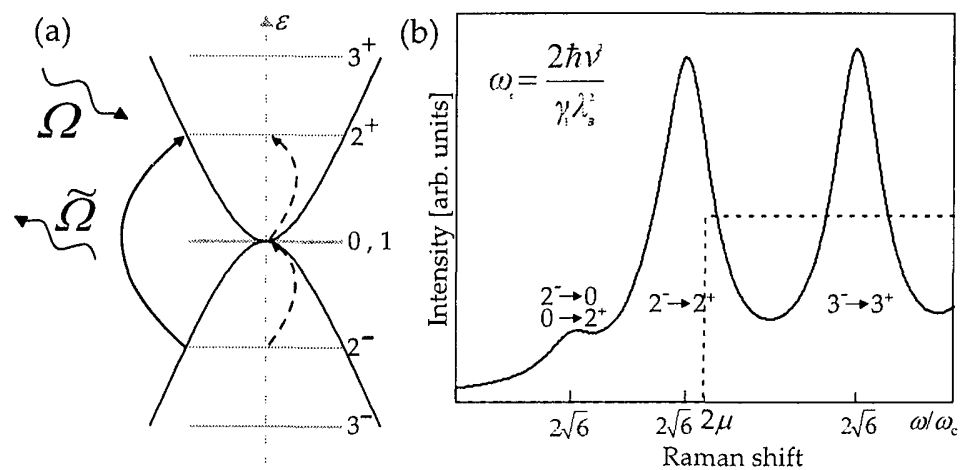

Figure 5.2: (a) Schematic drawing of allowed inter-LL transitions accompanying the Raman scattering. The solid (dashed) line represents the first dominant (weaker) transition $2^{-} \leftarrow 2^{+}$(pair $2^{-} \leftarrow 0$ and $0 \leftarrow 2^{+}$). (b) The low-energy electronic contribution to the Raman spectrum in bilayer graphene. The solid (dashed) line represents the spectrum in the presence (absence) of an external magnetic field and chemical potential $\mu=0(\mu \neq 0)$. For the spectrum in a magnetic field, corresponding inter-LL transitions have been attributed to each peak.

Here, we use Lorentzian $\gamma(x)=\pi^{-1} \Gamma /\left(x^{2}+\Gamma^{2}\right)$ with a width specified by $\Gamma$ to model the broadening of Landau levels. The term $\delta g(\omega)$ describes the spectral density of the $(n \mp 1)^{-} \rightarrow(n \pm 1)^{+}$transitions, which is a correction to the dominant contribution due to the $n^{-} \rightarrow n^{+}$transitions given by the first term on the right hand side of Eq. (5.14).

An example of the low-energy electronic contribution to the Raman spectrum in the neutral bilayer in strong magnetic field is shown with a solid line in Fig. 5.2(b). The dominant features are peaks due to the $n^{-} \rightarrow n^{+}$transitions with the first being the $2^{-} \rightarrow 2^{+}$transition. Note that within the LL indexing scheme applied here, indices 0 and 1 are only used to denote one valley-degenerate level each (no $\alpha$ index is needed). The quantum efficiency of a single $n^{-} \rightarrow n^{+}$peak in Fig. 5.2(b) is approximately

$$
I_{n^{-} \rightarrow n^{+}} \approx\left(\frac{v^{2}}{c^{2}} \frac{e^{2} / \lambda_{B}}{\epsilon_{0} \pi \Omega}\right)^{2}=\frac{v^{4} e^{5} B}{\pi^{2} c^{4} \epsilon_{0}^{2} \hbar \Omega^{2}}
$$

per incoming photon, which at the field $B \sim 10 \mathrm{~T}$ gives $I_{n^{-} \rightarrow n^{+}} \sim 10^{-12}$ for $\Omega \sim 1 \mathrm{eV}$ 


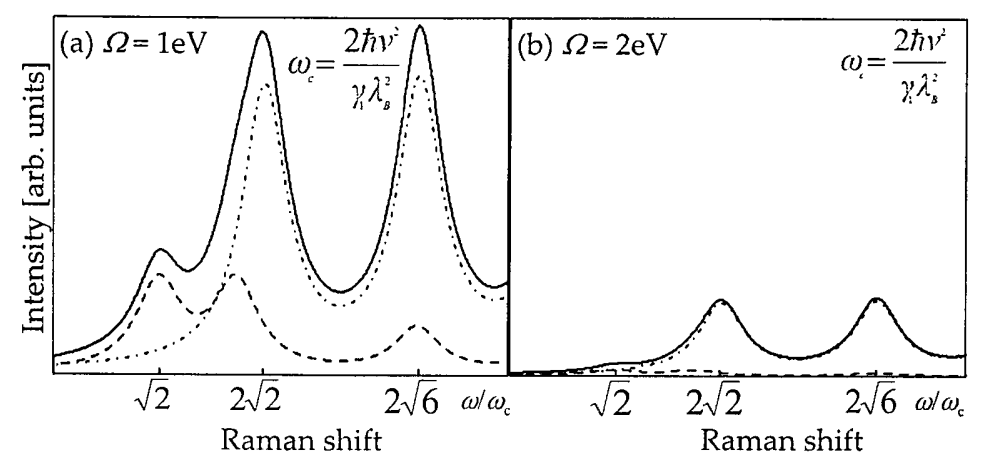

Figure 5.3: Comparison of electronic contributions to the Raman spectra in neutral bilayer graphene for two different energies of incoming photons: (a) $\Omega=1 \mathrm{eV},(\mathrm{b}) \Omega=2 \mathrm{eV}$. For each case, total spectral density $g(\omega)$ and contributions due to the $n^{-} \rightarrow n^{+}$and $(n \pm 1)^{-} \rightarrow(n \mp 1)^{+}$modes are shown in the solid, dotdashed and dashed line, respectively. Intensity scale is the same on (a) and (b); values of the parameters used: $v=10^{6} \mathrm{~m} / \mathrm{s}, \gamma_{1}=0.4 \mathrm{eV}, B=10 \mathrm{~T}$, and $\Gamma=0.012 \mathrm{eV}$.

photons, comparable to similar transitions in monolayer graphene [152].

A weaker feature in Fig. 5.2(b) is the first and the only visible $(n \mp 1)^{-} \rightarrow$ $(n \pm 1)^{+}$peak due to both $2^{-} \rightarrow 0$ and $0 \rightarrow 2^{+}$transitions, positioned to the left of the $2^{-} \rightarrow 2^{+}$peak. The quantum efficiencies of the $(n \pm 1)^{-} \rightarrow(n \mp 1)^{+}$transitions are smaller by the factor $\left(\frac{\gamma_{1}}{\Omega}\right)^{2}$ in comparison to the $n^{-} \rightarrow n^{+}$transitions. This is different from the monolayer graphene case, where the corresponding ratio between quantum efficiencies of $(n \pm 1)^{-} \rightarrow(n \mp 1)^{+}$and $n^{-} \rightarrow n^{+}$transitions is $\left(\frac{\omega}{\Omega}\right)^{2}$, much smaller than for the bilayer. The term $\delta g(\omega)$ can be further emphasized by changing the energy of incoming photons $\Omega$. Shown in Figs. 3(a) and $3(b)$, is a comparison of the total spectral density $g(\omega)$ and contributions due to each mode separately, for two different energies of incoming photons, $\Omega=$ $2 \mathrm{eV}$ and $\Omega=1 \mathrm{eV}$. The intensity scale is the same on both figures and in each case, the total spectral density $g(\omega)$, the contributions due to the $n^{-} \rightarrow n^{+}$and $(n \pm 1)^{-} \rightarrow(n \mp 1)^{+}$modes are shown in the solid, dot-dashed and dashed line, respectively. The dominant contribution, resulting from the Raman scattering accompanied by the $n^{-} \rightarrow n^{+}$electronic transitions, is proportional to the inverse square of the incoming photon energy $\Omega$. Therefore, two peaks drawn with dot-dashed lines are roughly four times smaller on the right figure. The spectral 
density of the $(n \pm 1)^{-} \rightarrow(n \mp 1)^{+}$transitions is smaller by a further factor $\left(\frac{\gamma_{1}}{\Omega}\right)^{2}$ in comparison to the $n^{-} \rightarrow n^{+}$transitions. Hence, this contribution, shown with dashed lines, is close to zero on the right figure, while on the left, the first of the two smaller peaks corresponding to symmetric transitions $2^{-} \rightarrow 0$, $0 \rightarrow 2^{+}$and $3^{-} \rightarrow 1,1 \rightarrow 3^{+}$is still visible in the total spectral density. Because of the contrasting polarization factors in Eq. (5.14), contributions of different modes, $n^{-} \rightarrow n^{+}$or $(n \pm 1)^{-} \rightarrow(n \mp 1)^{+}$, to the total spectral density could be separated using polarizers. If the polarizers were set as to collect only photons with circular polarization identical to that of the incoming photons, then the $n^{-} \rightarrow n^{+}$contribution would be measured. However, if only the photons with polarization opposite to the polarization of the incoming beam were detected, the $(n \mp 1)^{-} \rightarrow(n \pm 1)^{+}$contribution would be determined.

Increasing the filling factor leads first to the $2^{-} \rightarrow 0$ and $3^{-} \rightarrow 1$ transitions being blocked when LLs with $n=0$ and $n=1$ are completely filled. Therefore, the height of the two corresponding $(n \pm 1)^{-} \rightarrow(n \mp 1)^{+}$peaks is halved (transitions $0 \rightarrow 2^{+}$and $1 \rightarrow 3^{+}$are still allowed). Next to disappear are the first $n^{-} \rightarrow n^{+}$peak, that is $2^{-} \rightarrow 2^{+}$, and the remains of the first $(n \pm 1)^{-} \rightarrow(n \mp 1)^{+}$peak, (due to the $0 \rightarrow 2^{+}$transition) because of the filled LL $2^{+}$. Complete filling of each following Landau level results in the disappearance of the next $n^{-} \rightarrow n^{+}$and $(n \pm 1)^{-} \rightarrow(n \mp 1)^{+}$peaks. 


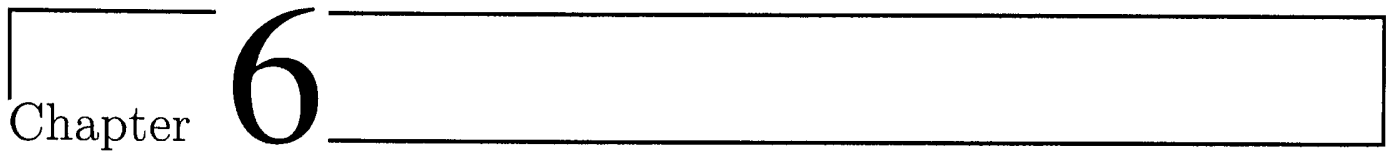

\section{Conclusions}

In the previous chapters, we developed theories based on the tight-binding model for $\pi$ electrons, in order to describe the angle-resolved photoemission, magneto-optical absorption and electronic Raman spectra of bilayer graphene. The results for the first two can be compared to experimental spectra. Specifically, the constant-energy ARPES maps found in literature (in particular in the supporting on-line material to the article by Ohta and co-workers [55]), closely resemble those shown in the Fig. 3.3, 3.4 and 3.6. The presence of the asymmetry can be deduced from the experimental spectra due to the appearance of the gap in the spectrum. The experimentally obtained ARPES spectra were also detailed enough for the magnitude of the direct interlayer coupling $\gamma_{1}$ to be extracted. Unfortunately, little experimental data does not allow testing of other predictions of our model, that is, whether the sign of $\gamma_{1}$ as well as magnitude and sign of $\gamma_{3}$ can be determined.

In the case of the magneto-optical absorption, the spectra shown in Figs. 4.3(c) and 4.8 can be compared to those predicted and experimentally measured for optical absorption $[69,85,88,95,158]$. The main feature - the peak corresponding to the onset of the transitions between the low-energy and split bands

- is similar in both cases and allows for an independent check on the value of the coupling $\gamma_{1}$. However, it is very difficult to comment on the accurateness of the main point of the considerations in Chapter 4 - the importance of the 
gate-induced interlayer asymmetry in the interpretation of the experimental results of the work of Henriksen et al. [63]. Although the presented theory suggests the presence of the gap, it is definitely not the only factor responsible for the observed deviations from the 'neutral bilayer' model. In fact, some of the many-body theories show significantly better agreement with the experiment [146, 147].

The situation is different for the electronic Raman spectroscopy. Although inelastic scattering of light is a widely used experimental technique for characterisation of carbon materials and has been employed for graphene systems specifically to investigate not only the electron-phonon coupling but also number of layers, disorder or doping level, the electronic contribution to the Raman spectra has not yet been examined in detail. Therefore, theory presented in Chapter 5 goes one step beyond being tested by comparison to available measurements. The absolute numbers predicted for the quantum efficiencies of the inelastic light scattering accompanied by electron-hole excitations suggest that some of the features discussed may be observable. In our opinion, in the light of the complications in the interpretation of the experimental results of experiments of the type of the one by Henriksen and co-workers, examination of the ERS spectra in an external magnetic field would provide an important additional way of investigating the Landau level structure of bilayer graphene. This would also give more insight on the usefulness and limits of the tight-binding approach for graphene systems.

Note, that in all three cases presented, it was important to choose as the starting point for the theory, the four-band model. The two-band approximation, neglecting the influence of the high-energy states, does not capture essential features of the spectra discussed in any of the chapters. For ARPES, it cannot describe the asymmetry in the intensity between the two-peak pattern (see Fig. 3.3 and discussion in Sec. 3.3.2) even at relatively low energies. The high- 
energy bands are essential in order to obtain the self-consistent values of the interlayer asymmetry both in the absence and presence of the magnetic field. The two-band approximation, for obvious reasons, also cannot describe the inter-Landau-level transitions between the low-energy and split bands caused by absorption of incoming photons. Finally, for inelastic scattering of photons, we showed that the ERS scattering amplitude for experimentally relevant energies of the incoming beam cannot be properly described within the two-band approximation alone. Due to the importance of the high-energy bands in the two-step processes, proper results can only be obtained within the full fourband model. All those arguments show how carefully any problem related to the electronic structure of bilayer graphene has to be considered before only the two-band approximation is used to predict or explain its results. Good understanding of the physical processes involved is required if the theoretical model is to be correct and useful. 


\section{Bibliography}

[1] J. D. Bernal, The structure of graphite, Proceedings of the Royal Society A 106 (1924), 749.

[2] P. R. Wallace, The band theory of graphite, Physical Review 71 (1947), 622.

[3] J. W. McClure, Band structure of graphite and de Haas-van Alphen effect, Physical Review 108 (1957), 612.

[4] J. C.Slonczewski and P. R. Weiss, Band structure of graphite, Physical Review 109 (1958), 272.

[5] H. W. Kroto, J. R. Heath, S. C. O'Brien, R. F. Curl, and R. E. Smalley, C60: Buckminsterfullerene, Nature 318 (1985), 162.

[6] S. Ijima, Helical microtubules of graphite carbon, Nature 354 (1991), 56.

[7] S. Ijima and T. Ichihashi, Single-shell carbon nanotubes of 1-nm diameter, Nature 363 (1993), 603.

[8] K. S. Novoselov, A. K. Geim, S. V. Morozov, D. Jiang, Y. Zhang, S. V. Dubonos, I. V. Grigorieva, and A. A. Firsov, Electric field effect in atomically thin carbon films, Science 306 (2004), 666.

[9] K. S. Novoselov, D. Jiang, T. Booth, V. V. Khotkevich, S. V. Morozov, and 
A. K. Geim, Two-dimensional atomic crystals, Proceedings of the National Academy of Sciences USA 102 (2005), 10451.

[10] K. S. Novoselov, A. K. Geim, S. V. Morozov, D. Jiang, M. I. Katsnelson, I. V. Grigorieva, S. V. Dubonos, and A. A. Firsov, Two-dimensional gas of massless dirac fermions in graphene, Nature 438 (2005), 197.

[11] Y. Zhang, Y. W. Tan, H. L. Stormer, and P. Kim, Experimental observation of quantum Hall effect and Berry's phase in graphene, Nature 438 (2005), 201.

[12] M. I. Katsnelson, Graphene: carbon in two dimensions, Materials Today 10 (2007), 20.

[13] A. K. Geim and K. S. Novoselov, The rise of graphene, Nature Materials 6 (2007), 183.

[14] M. I. Katsnelson and K. S. Novoselov, Graphene: New bridge between condensed matter physics and quantum electrodynamics, Solid State Communications 143 (2007), 3.

[15] A. K. Geim, Graphene: Status and prospects, Science 324 (2009), 1530.

[16] D. S. L. Abergel, V. Apalkov, J. Berashevich, K. Ziegler, and T. Chakraborty, Properties of graphene: a theoretical perspective, Advances in Physics 59 (2010), 261.

[17] J. Hass, W. A. de Heer, and E. H. Conrad, The growth and morphology of epitaxial multilayer graphene, Journal of Physics: Condensed Matter 20 (2008), 323202.

[18] J. Wintterlin and M.-L. Bocquet, Graphene on metal surfaces, Surface Science 603 (2009), 1841. 
[19] M. Orlita and M. Potemski, Dirac electronic states in graphene systems: optical spectroscopy studies, Semiconductor Science and Technology 25 (2010), 063001.

[20] A. C. Ferrari, Raman spectroscopy of graphene and graphite: Disorder, electronphonon coupling, doping and nonadiabatic effects, Solid State Communications 143 (2007), 47.

[21] N. Ferralis, Probing mechanical properties of graphene with Raman spectroscopy, Journal of Materials Science 97 (2010), 5135.

[22] A. Bostwick, K. V. Emtsev, K. Horn, E. Huwald, L. Ley, J. L. McChesney, T. Ohta, J. Riley, E. Rotenberg, F. Speck, and T. Seyller, Photoemission studies of graphene on SiC: Growth, interface, and electronic structure, Advances in Solid State Physics 47 (2008), 159.

[23] K. S. Novoselov, S. V. Morozov, T. M. G. Mohinddin, L. A. Ponomarenko, D. C. Elias, R. Yang, I. I. Barbolina, P. Blake, T. J. Booth, D. Jiang, J. Giesbers, E. W. Hill, and A. K. Geim, Electronic properties of graphene, Physica Status Solibi B 244 (2007), 4106.

[24] C. W. J. Beenakker, Andreev reflection and Klein tunneling in graphene, Reviews of Modern Physics 80 (2008), 1337.

[25] A. H. Castro Neto, F. Guinea, N. M. R. Peres, K. S. Novoselov, and A. K. Geim, The electronic properties of graphene, Reviews of Modern Physics 81 (2009), 109.

[26] A. Bostwick, J. L. McChesney, T. Ohta, E. Rotenberg, T. Seyller, and K. Horn, Experimental studies of the electronic structure of graphene, Progress in Surface Science 84 (2009), 380.

[27] N. M. R. Peres, The transport properties of graphene: an introduction, arXiv: 1007.2849 (2010). 
[28] S. Das Sarma, S. Adam, E. H. Hwang, and E. Rossi, Electronic transport in two dimensional graphene, arXiv: 1003.4731 (2010).

[29] C. Oshima and A. Nagashima, Ultra-thin epitaxial films of graphite and hexagonal boron nitride on solid surfaces, Journal of Physics: Condensed Matter 9 (1997), 1.

[30] A. Charrier, A. Coati, T. Argunova, F. Thibaudau, Y. Garreau, R. Pinchaux, I. Forbeaux, J.-M. Debever, M. Sauvage-Simkin, and J.-M. Themlin, Solid-state decomposition of silicon carbide for growing ultra-thin heteroepitaxial graphite films, Journal of Applied Physics 92 (2002), 2479.

[31] C. Berger, Z. Song, T. Li, X. Li, A. Y. Ogbazghi, R. Feng, Z. Dai, A. N. Marchenkov, E. H. Conrad, P. N. First, and W. A. de Heer, Ultrathin epitaxial graphite: $2 d$ electron gas properties and a route toward graphene-based nanoelectronics, Journal of Physical Chemistry B 108 (2004), 19912.

[32] C. Berger, Z. Song, X. Li, X. Wu, N. Brown, C. Naud, D. Mayou, T. Li, J. Hass, A. N. Marchenkov, E. H. Conrad, P. N. First, and W. A. de Heer, Electronic confinement and coherence in patterned epitaxial graphene, Science 312 (2006), 1191.

[33] E. Rollings, G.-H. Gweon, S. Y. Zhou, B. S. Mun, J. L. McChesney, B. S. Hussain, A. V. Federov, P. N. First, W. A. de Heer, and A. Lanzara, Synthesis and characterization of atomically thin graphite films on a silicon carbide substrate, Journal of Physics and Chemistry of Solids 67 (2006), 2172.

[34] M. Sprinkle, D. Siegel, Y. Hu, J. Hicks, A. Tejede, A. Taleb-Ibrahimi, P. Le Fèvre, F. Bertran, S. Vizzini, H. Enriquez, S. Chiang, P. Soukiassian, C. Berger, W. A. de Heer, A. Lanzara, and E. H. Conrad, First direct observation of a nearly ideal graphene band structure, Physical Review Letters 103 (2009), 226803. 
[35] Y. S. Dedkov, M. Fonin, and C. Laubschat, A possible source of spin-polarized electrons: The inert graphene/Ni(111) system, Applied Physics Letters 92 (2008), 052506.

[36] Y. S. Dedkov, M. Fonin, U. Rüdiger, and C. Laubschat, Rashba effect in the graphene/Ni(111) system, Physical Review Letters 100 (2008), 107602.

[37] A. Grüneis and D. V. Vyalikh, Tunable hybridization between electronic states of graphene and a metal surface, Physical Review B 77 (2008), 193401.

[38] A. Grüneis, K. Kummer, and D. V. Vyalikh, Dynamics of graphene growth on a metal surface: a time-dependent photoemission study, New Journal of Physics 11 (2009), 073050.

[39] I. Pletikosić, M. Kralj, P. Pervan, R. Brako, J. Coraux, A. T. N'Diaye, C. Busse, and T. Michely, Dirac cones and minigaps for graphene on $\operatorname{Ir}(111)$, Physical Review Letters 102 (2009), 056808.

[40] A. L. Vázquez de Parga, F. Calleja, B. Borca, M. C. G. Passegi Jr., J. J. Hinarejos, F. Guinea, and R. Miranda, Periodically rippled graphene: growth and spatially resolved electronic structure, Physical Review Letters 100 (2008), 056807.

[41] P. W. Sutter, J.-I. Flege, and E. A. Sutter, Epitaxial graphene on ruthenium, Nature Materials 7 (2008), 406.

[42] C. Enderlein, Y. S. Kim, A. Bostwick, E. Rotenberg, and K. Horn, The formation of an energy gap in graphene on ruthenium by controlling the interface, New Journal of Physics 12 (2010), 033014.

[43] Y. Lee, S. Bae, H. Jang, S. Jang, S.-E. Zhu, S. H. Sim, Y. I. Song, B. H. Hong, and J.-H. Ahn, Wafer-scale synthesis and transfer of graphene films, Nano Letters 10 (2010), 490. 
[44] K. S. Kim, Y. Zhao, H. Jang, S. Y. Lee, J. M. Kim, K. S. Kim, J.-H. Ahn, P. Kim, J.-Y. Choi, and B. H. Hong, Large-scale pattern growth of graphene films for stretchable transparent electrodes, Nature 457 (2009), 706.

[45] A. Reina, S. Thiele, X. Jia, S. Bhaviripudi, M. S. Dresselhaus, J. A. Schaefer, and J. Kong, Growth of large-area single- and bi-layer graphene by controlled carbon precipitation on polycrystalline ni surfaces, Nano Research 2 (2009), 509.

[46] H. J. Park, J. Meyer, S. Roth, and V. Skakalova, Growth and properties of few-layer graphene prepared by chemical vapor deposition, Carbon 48 (2010), 1088.

[47] Z.-Y. Juang, C.-Y. Wu, A.-Y. Lu, C.-Y. Su, K.-C. Leou, F.-R. Chen, and C.-H. Tsai, Graphene synthesis by chemical vapor deposition and transfer by a roll-to-roll process, Carbon 48 (2010), 3169.

[48] M. Xu, D. Fujita, K. Sagisaka, E. Watanabe, and N. Hanagata, Single-layer graphene nearly 100\% covering an entire substrate, arXiv: 1006.5085 (2010).

[49] J.-C. Charlier, J.-P. Michenaud, and X. Gonze, First-principles study of the electronic properties of simple hexagonal graphite, Physical Review B 46 (1992), 4531.

[50] M. S. Dresselhaus and G. Dresselhaus, Intercalation compounds of graphite, Advances in Physics 30 (1981), 139.

[51] E. McCann and V. I. Fal'ko, Landau level degeneracy and quantum hall effect in a graphite bilayer, Physical Review Letters 96 (2006), 086805.

[52] K. Kechedzhi, E. McCann, V. Fal'ko, and B. Altshuler, Influence of trigonal warping on interference effects in bilayer graphene, Physical Review Letters 98 (2007), 176806. 
[53] K. Kechedzhi, E. McCann, V. Fal'ko, H. Suzuura, T. Ando, and B. Altshuler, Weak localization in monolayer and bilayer graphene, European Physical Journal Special Topics 148 (2007), 39.

[54] K. S. Novoselov, E. McCann, S. V. Morozov, V. I. Fal'ko, M. I. Katsnelson, U. Zeitler, D. Jiang, F. Schedin, and A. K. Geim, Unconventional quantum Hall effect and Berry's phase of 2pi in bilayer graphene, Nature Physics 2 (2006), 177.

[55] T. Ohta, A. Bostwick, T. Seyller, K. Horn, and E. Rotenberg, Controlling the electronic structure of bilayer graphene, Science 313 (2006), 951.

[56] A. Bostwick, T. Ohta, T. Seyller, K. Horn, and E. Rotenberg, Quasiparticle dynamics in graphene, Nature Physics 3 (2007), 36.

[57] S. Y. Zhou, G.-H. Gweon, A. V. Fedorov, P. N. First, W. A. de Heer, D.H. Lee, F. Guinea, A. H. Castro Neto, and A. Lanzara, Substrate-induced bandgap opening in epitaxial graphene, Nature Materials 6 (2007), 770.

[58] M. Mucha-Kruczyński, O. Tsyplyatyev, A. Grishin, E. McCann, V. I. Fal'ko, A. Bostwick, and E. Rotenberg, Characterization of graphene through anisotropy of constant-energy maps in angle-resolved photoemission, Physical Review B 77 (2008), 195403.

[59] M. L. Sadowski, G. Martinez, M. Potemski, C. Berger, and W. A. de Heer, Landau level spectroscopy of ultrathin graphite layers, Physical Review Letters 97 (2006), 266405.

[60] Z. Jiang, E. A. Henriksen, L. C. Tung, Y.-J. Wang, M. E. Schwartz, M. Y. Han, P. Kim, and H. L. Stormer, Infrared spectroscopy of Landau levels of graphene, Physical Review Letters 98 (2007), 197403.

[61] R. S. Deacon, K.-C. Chuang, R. J. Nicholas, K. S. Novoselov, and A. K. 
Geim, Cyclotron resonance study of the electron and hole velocity in graphene monolayers, Physical Review B 76 (2007), 081406(R).

[62] M. L. Sadowski, G. Martinez, M. Potemski, C. Berger, and W. A. de Heer, Magnetospectroscopy of epitaxial few-layer graphene, Solid State Communications 143 (2007), 123.

[63] E. A. Henriksen, Z. Jiang, L.-C. Tung, M. E. Schwartz, M. Takita, Y.-J. Wang, P. Kim, and H. L. Stormer, Cyclotron resonance in bilayer graphene, Physical Review Letters 100 (2008), 087403.

[64] P. Płochocka, C. Faugeras, M. Orlita, M. L. Sadowski, G. Martinez, M. Potemski, M. O. Goerbig, J.-N. Fuchs, C. Berger, and W. A. de Heer, High-energy limit of massless Dirac fermions in multilayer graphene using magneto-optical transmission spectroscopy, Physical Review Letters 100 (2008), 087401.

[65] M. Orlita, C. Faugeras, P. Plochocka, P. Neugebauer, G. Martinez, D. K. Maude, A.-L. Barra, M. Sprinkle, C. Berger, W. A. de Heer, and M. Potemski, Approaching the Dirac point in high mobility multi-layer epitaxial graphene, Physical Review Letters 101 (2008), 267601.

[66] P. Neugebauer, M. Orlita, C. Faugeras, A.-L. Barra, and M. Potemski, How perfect can graphene be?, Physical Review Letters 103 (2009), 136403.

[67] E. A. Henriksen, P. Cadden-Zimansky, Z. Jiang, Z. Q. Li, L.-C. Tung, M. E. Schwartz, M. Takita, Y.-J. Wang, P. Kim, and H. L. Stormer, Interactioninduced shift of the cyclotron resonance of graphene using infrared spectroscopy, Physical Review Letters 104 (2010), 067404.

[68] A. M. Witowski, M. Orlita, R. Stepniewski, A. Wysmolek, J. M. Baranowski, W. Strupinski, C. Faugeras, G. Martinez, and M. Potemski, 
Quasi-classical cyclotron resonance of Dirac fermions in highly doped graphene, arXiv: 1007.4153 (2010).

[69] D. S. L. Abergel and V. I. Fal'ko, Optical and magneto-optical far-infrared properties of bilayer graphene, Physical Review B 75 (2007), 155430.

[70] D. S. L. Abergel, E. McCann, and V. I. Fal'ko, QHE and far infra-red properties of bilayer graphene in a strong magnetic field, European Physical Journal Special Topics 148 (2007), 105.

[71] M. Mucha-Kruczyński, D. S. L. Abergel, E. McCann, and V. I. Fal'ko, On spectral properties of bilayer graphene: the effect of an SiC substrate and infrared magneto-spectroscopy, Journal of Physics: Condensed Matter 21 (2009), 344206.

[72] E. McCann, Asymmetry gap in the electronic band structure of bilayer graphene, Physical Review B 74 (2006), 161403(R).

[73] M. Mucha-Kruczyński, E. McCann, and V. I. Fal'ko, Influence of interlayer asymmetry on magneto-spectroscopy of bilayer graphene, Solid State Communications 149 (2009), 1111.

[74] M. V. Klein, Electronic Raman scattering, in M. Cardona (Editor), Light Scattering in Solids, volume I, Springer, Berlin/Heidelberg, 1983.

[75] M. Mucha-Kruczyński, O. Kashuba, and V. I. Fal'ko, Spectral features due to inter-Landau-level transitions in the Raman spectrum of bilayer graphene, Physical Review B (2010).

[76] J. W. McClure, Theory of diamagnetism of graphite, Physical Review 119 (1960), 606.

[77] R. Saito, G. Dresselhaus, and M. S. Dresselhaus, Physical properties of Carbon Nanotubes, Imperial College Press, London, 1998. 
[78] C. Bena and G. Montambaux, Remarks on the tight-binding model of graphene, New Journal of Physics 11 (2009), 095003.

[79] F. Varchon, R. Feng, J. Hass, X. Li, B. Ngoc Nguyen, C. Naud, P. Mallet, J.-Y. Veuillen, C. Berger, E. H. Conrad, and L. Magaud, Electronic structure of epitaxial graphene layers on SiC: Effect of the substrate, Physical Review Letters 99 (2007), 126805.

[80] S. B. Trickey, F. Müller-Plathe, and G. H. F. Diercksen, Interplanar binding and lattice relaxation in a graphite dilayer, Physical Review B 45 (1992), 4460.

[81] H. Ajiki and T. Ando, Electronic states of carbon nanotubes, Journal of the Physical Society of Japan 62 (1993), 1255.

[82] T. Ando, Theory of electronic states and transport in carbon nanotubes, Journal of the Physical Society of Japan 74 (2005), 777.

[83] J. M. Luttinger and W. Kohn, Motion of electrons and holes in perturbed periodic fields, Physical Review 97 (1955), 869.

[84] D. P. DiVincenzo and E. J. Mele, Self-consistent effective-mass theory for intralayer screening in graphite intercalation compounds, Physical Review B 29 (1984), 1685.

[85] L. M. Zhang, Z. Q. Li, D. N. Basov, M. M. Fogler, Z. Hao, and M. C. Martin, Determination of the electronic structure of bilayer graphene from infrared spectroscopy, Physical Review B 78 (2008), 235408.

[86] A. B. Kuzmenko, E. van Heumen, D. van der Marel, P. Lerch, P. Blake, K. S. Novoselov, and A. K. Geim, Infrared spectroscopy of electronic bands in bilayer graphene, Physical Review B 79 (2009), 115441.

[87] A. B. Kuzmenko, I. Crassee, D. van der Marel, P. Blake, and K. S. Novoselov, Determination of the gate-tunable band gap and tight-binding pa- 
rameters in bilayer graphene using infrared spectroscopy, Physical Review B 80 (2009), 165406.

[88] Z. Q. Li, E. A. Henriksen, Z. Jiang, Z. Hao, M. C. Martin, P. Kim, H. L. Stormer, and D. N. Basov, Band structure asymmetry of bilayer graphene revealed by infrared spectroscopy, Physical Review Letters 102 (2009), 037403.

[89] L. M. Malard, J. Nilsson, D. C. Elias, J. C. Brant, F. Plentz, E. S. Alves, A. H. Castro Neto, and M. A. Pimenta, Probing the electronic structure of bilayer graphene by Raman scattering, Physical Review B 76 (2007), 201401(R).

[90] J. Yan, E. A. Henriksen, P. Kim, and A. Pinczuk, Observation of anomalous phonon softening in bilayer graphene, Physical Review Letters 101 (2008), 136804.

[91] A. Das, B. Chakraborty, S. Piscanec, S. Pisana, A. K. Sood, and A. C. Ferrari, Phonon renormalization in doped bilayer graphene, Physical Review B 79 (2009), 155417.

[92] D. L. Mafra, L. M. Malard, S. K. Doorn, H. Htoon, J. Nilsson, A. H. Castro Neto, and M. A. Pimenta, Observation of the Kohn anomaly near the K point of bilayer graphene, Physical Review B 80 (2009), 241414(R).

[93] J. B. Oostinga, H. B. Heersche, X. L. Liu, A. F. Morpurgo, and L. M. K. Vandersypen, Gate-induced insulating state in bilayer graphene devices, Nature Materials 7 (2008), 151.

[94] Y. Zhang, T.-T. Tang, C. Girit, Z. Hao, M. C. Martin, A. Zettl, M. F. Crommie, Y. R. Shen, and F. Wang, Direct observation of a widely tunable bandgap in bilayer graphene, Nature 459 (2009), 820.

[95] K. F. Mak, C. H. Lui, J. Shan, and T. F. Heinz, Observation of an electricfield-induced band gap in bilayer graphene by infrared spectroscopy, Physical Review Letters 102 (2009), 256405. 
[96] M. I. Katsnelson, K. S. Novoselov, and A. K. Geim, Chiral tunnelling and the Klein paradox in graphene, Nature Physics 2 (2006), 620.

[97] M. I. Katsnelson, Minimal conductivity in bilayer graphene, European Physical Journal B 52 (2006), 151.

[98] E. McCann, D. S. L. Abergel, and V. I. Fal'ko, Electrons in bilayer graphene, Solid State Communications 143 (2007), 110.

[99] M. Mucha-Kruczyński, E. McCann, and V. I. Fal'ko, Electron-hole asymmetry and energy gaps in bilayer graphene, Semiconductor Science and Technology 25 (2010), 033001.

[100] I. Lifshitz, Anomalies of electron characteristics of a metal in the high pressure region, Soviet Physics Journal of Experimental and Theoretical Physics 11 (1960), 1130.

[101] H. Hertz, Ueber einen Einfluss des ultravioletten Lichtes auf die electrische Entladung, Annalen der Physik 267 (1887), 983.

[102] A. Einstein, Über einen die Erzeugung und Verwandlung des Lichtes betreffenden heuristischen Gesichtspunkt, Annalen der Physik 322 (1905), 132.

[103] P. M. Williams, The direct evaluation of electronic band structures of layered solids using angle-resolved photoemission, Il Nuovo Cimento 38B (1977), 216.

[104] I. T. McGovern, W. Eberhardt, E. W. Plummer, and J. E. Fischer, The bandstructures of graphite and graphite intercalation compounds as determined by angle resolved photoemission using synchrotron radiation, Physica 99B (1980), 415.

[105] W. Eberhardt, I. T. McGovern, E. W. Plummer, and J. E. Fisher, Chargetransfer and non-rigid-band effects in the graphite compound $\mathrm{LiC}_{6}$, Physical Review Letters 44 (1980), 200. 
[106] A. R. Law, J. J. Barry, and H. P. Hughes, Angle-resolved photoemission and secondary electron emission from single-crystal graphite, Physical Review B 28 (1983), 5332.

[107] T. Takahashi, H. Tokailin, and S. Sagawa, Electronic band structure of graphite studied by highly angle-resolved ultraviolet photoelectron spectroscopy, Solid State Communications 52 (1984), 765.

[108] A. Santoni, L. J. Terminello, F. J. Himpsel, and T. Takahashi, Mapping the Fermi surface of graphite with a display-type photoelectron spectrometer, Applied Physics A 52 (1991), 299.

[109] E. L. Shirley, L. J. Terminello, A. Santoni, and F. J. Himpsel, Brillouinzone-selection effects in graphite photoelectron angular distributions, Physical Review B 51 (1995), 13614.

[110] A. Bostwick, T. Ohta, J. L. McChesney, T. Seyller, K. Horn, and E. Rotenberg, Renormalization of graphene bands by many-body interactions, Solid State Communications 143 (2007), 63.

[111] A. Bostwick, T. Ohta, J. L. McChesney, T. Seyller, K. Horn, and E. Rotenberg, Band structure and many body effects in graphene, European Physical Journal Special Topics 148 (2007), 5.

[112] C.-H. Park, F. Giustino, M. L. Cohen, and S. G. Louie, Velocity renormalization and carrier lifetime in graphene from the electron-phonon interaction, Physical Review Letters 99 (2007), 086804.

[113] M. Calandra and F. Mauri, Electron-phonon coupling and electron self-energy in electron-doped graphene: Calculation of angular-resolved photoemission spectra, Physical Review B 76 (2007), 205411.

[114] W.-K. Tse and S. Das Sarma, Phonon-induced many-body renormalization 
of the electronic properties of graphene, Physical Review Letters 99 (2007), 236802.

[115] M. Polini, R. Asgari, G. Borghi, Y. Barlas, T. Pereg-Barnea, and A. H. MacDonald, Plasmons and the spectral function of graphene, Physical Review B 77 (2008), 081411(R).

[116] P. E. Trevisanutto, C. Giorgetti, L. Reining, M. Ladisa, and V. Olevano, $A b$ initio GW many-body effects in graphene, Physical Review Letters 101 (2008), 226405.

[117] C.-H. Park, F. Giustino, C. D. Spataru, M. L. Cohen, and S. G. Louie, Angleresolved photoemission spectra of graphene from first-principles calculations, Nano Letters 9 (2009), 4234.

[118] A. Bostwick, T. Ohta, J. L. McChesney, K. V. Emtsev, T. Seyller, K. Horn, and E. Rotenberg, Symmetry breaking in few layer graphene films, New Journal of Physics 9 (2007), 385.

[119] E. Rotenberg, A. Bostwick, T. Ohta, J. L. McChesney, T. Seyller, and K. Horn, Origin of the energy bandgap in epitaxial graphene [comment on Zhou et al., [57]], Nature Materials 7 (2007), 258.

[120] S. Y. Zhou, G.-H. Gweon, A. V. Fedorov, P. N. First, W. A. de Heer, D.-H. Lee, F. Guinea, A. H. Castro Neto, and A. Lanzara, Authors' response [to the comment by Rotenberg et al., [119]], Nature Materials 7 (2007), 259.

[121] S. Y. Zhou, D. A. Siegel, A. V. Fedorov, and A. Lanzara, Metal to insulator transition in epitaxial graphene induced by molecular doping, Physical Review Letters 101 (2008), 086402.

[122] C. Coletti, C. Riedl, D. S. Lee, B. Krauss, L. Patthey, K. von Klitzing, J. H. Smet, and U. Starke, Charge neutrality and band-gap tuning of epitaxial graphene on SiC by molecular doping, Physical Review B 81 (2010), 235401. 
[123] T. Ohta, A. Bostwick, J. L. McChesney, T. Seyller, K. Horn, and E. Rotenberg, Interlayer interaction and electronic screening in multilayer graphene investigated with angle-resolved photoemission spectroscopy, Physical Review Letters 98 (2007), 206802.

[124] F. J. Himpsel, Angle-resolved measurements of the photoemission of electrons in the study of solids, Advances in Physics 32 (1983), 1.

[125] A. Damascelli, Probing the electronic structure of complex systems by ARPES, Physica Scripta T109 (2004), 61.

[126] S. Y. Zhou, G.-H. Gweon, J. Graf, A. V. Fedorov, C. D. Spataru, R. D. Diehl, Y. Kopelevich, D.-H. Lee, S. G. Louie, and A. Lanzara, First direct observation of Dirac fermions in graphite, Nature Physics 2 (2006), 595.

[127] R. P. Feynman, The Feynman lectures on physics, volume III, AddisonWesley, Reading, 1963.

[128] L. Landau, Diamagnetismus der Metalle, Zeitschrift für Physik 64 (1930), 629.

[129] L. J. Sham, Electronic properties in semiconductor heterostructures, in P. Butcher, N. H. March, March, and M. P. Tosi (Editors), Physics of LowDimensional Semiconductor Structures, Plenum Press, New York, 1993.

[130] T. Chakraborty and P. Pietiläinen, The Quantum Hall Effects, SpringerVerlag, Berlin, 1995.

[131] N. d'Ambrumenil, Theory of the Quantum Hall Effect, in P. Butcher, N. H. March, and M. P. Tosi (Editors), Physics of Low-Dimensional Semiconductor Structures, Plenum Press, New York, 1993.

[132] Z. Jiang, Y. Zhang, Y.-W. Tan, H. L. Stormer, and P. Kim, Quantum Hall effect in graphene, Solid State Communications 143 (2007), 14. 
[133] J. C. Maan, Magneto-optical properties of semiconductor heterostructures, in P. Butcher, N. H. March, and M. P. Tosi (Editors), Physics of Low-Dimensional Semiconductor Structures, Plenum Press, New York, 1993.

[134] W. Kohn, Cyclotron resonance and de Haas-van Alphen oscillations of an interacting electron gas, Physical Review 123 (1961), 1242.

[135] M. Inoue, Landau levels and cyclotron resonance in graphite, Journal of the physical society of Japan 17 (1962), 808.

[136] O. P. Gupta and P. R. Wallace, Effect of trigonal warping on Landau-levels of graphite, Physica status solidi B 54 (1972), 53.

[137] K. Nakao, Landau-level structure and magnetic breakthrough in graphite, Journal of the Physical Society of Japan 40 (1976), 761.

[138] B. E. Feldman, J. Martin, and A. Yacoby, Broken-symmetry states and divergent resistance in suspended bilayer graphene, Nature Physics 5 (2009), 889.

[139] E. V. Castro, K. S. Novoselov, S. V. Morozov, N. M. R. Peres, J. M. B. Lopes dos Santos, J. Nilsson, F. Guinea, A. Geim, and A. H. Castro Neto, Biased bilayer graphene: Semiconductor with a gap tunable by the electric field effect, Physical Review Letters 99 (2007), 216802.

[140] H. Min, B. Sahu, S. K. Banerjee, and A. H. MacDonald, Ab initio theory of gate induced gaps in graphene bilayers, Physical Review B 75 (2007), 155115.

[141] F. Guinea, A. H. Castro Neto, and N. M. R. Peres, Electronic states and landau levels in graphene stacks, Physical Review B 73 (2006), 245426.

[142] J. M. Pereira Jr., F. M. Peeters, and P. Vasilopoulos, Landau levels and oscillator strength in a biased bilayer of graphene, Physical Review B 76 (2007), 115419. 
[143] T. Misumi and K. Shizuya, Electromagnetic response and pseudo-zero-mode landau levels of bilayer graphene in a magnetic field, Physical Review B 77 (2008), 195423.

[144] E. V. Castro, K. S. Novoselov, S. V. Morozov, N. M. R. Peres, J. M. B. Lopes dos Santos, J. Nilsson, F. Guinea, A. Geim, and A. H. Castro Neto, Electronic properties of a biased graphene bilayer, Journal of Physics: Condensed Matter 22 (2010), 175503.

[145] S. V. Kusminskiy, D. K. Campbell, and A. H. Castro Neto, Electron-electron interactions in graphene bilayers, Europhysics Letters 85 (2009), 58005.

[146] D. S. L. Abergel and T. Chakraborty, Long-range Coulomb interaction in bilayer graphene, Physical Review Letters 102 (2009), 056807.

[147] K. Shizuya, Many-body corrections to cyclotron resonance in monolayer and bilayer graphene, Physical Review B 81 (2010), 075407.

[148] G. Abstreiter, M. Cardona, and A. Pinczuk, Light scattering by free carrier excitations in semiconductors, in M. Cardona and G. Güntherodt (Editors), Light Scattering in Solids, volume IV, Springer, Berlin/Heidelberg, 1984.

[149] P. A. Wolff, Thomson and Raman scattering by mobile electrons in crystals, Physical Review Letters 16 (1966), 225.

[150] R. E. Slusher, C. K. N. Patel, and P. A. Fleury, Inelastic light scattering from Landau-level electrons in semiconductors, Physical Review Letters 18 (1967), 77.

[151] C. K. N. Patel and R. E. Slusher, Light scattering from electron plasmas in a magnetic field, Physical Review Letters 21 (1968), 1563.

[152] O. Kashuba and V. I. Fal'ko, Signature of electronic excitations in the Raman spectrum of graphene, Physical Review B 80 (2009), 241404(R). 
[153] A. C. Ferrari, J. C. Meyer, V. Scardaci, C. Casiraghi, M. Lazzeri, F. Mauri, S. Piscanec, D. Jiang, K. S. Novoselov, S. Roth, and A. K. Geim, Raman spectrum of graphene and graphene layers, Physical Review Letters 97 (2006), 187401.

[154] A. Gupta, G. Chen, P. Joshi, S. Tadigadapa, and P. C. Eklund, Raman scattering from high-frequency phonons in supported n-graphene layer films, Nano Letters 6 (2006), 2667.

[155] D. Graf, F. Molitor, K. Ensslin, C. Stampfer, A. Jungen, C. Hierold, and L. Wirtz, Spatially resolved Raman spectroscopy of single- and few-layer graphene, Nano Letters 7 (2007), 238.

[156] L. M. Malard, D. C. Elias, E. S. Alves, and M. A. Pimenta, Observation of distinct electron-phonon couplings in gated bilayer graphene, Physical Review Letters 101 (2008), 257401.

[157] Z. Ni, L. Liu, Y. Wang, Z. Zheng, L.-J. Li, T. Yu, and Z. Shen, G-band Raman double resonance in twisted bilayer graphene: Evidence of band splitting and folding, Physical Review B 80 (2009), 125404.

[158] E. J. Nicol and J. P. Carbotte, Optical conductivity of bilayer graphene with and without an asymmetry gap, Physical Review B 77 (2008), 155409. 